\title{
ELEVEN MULTIPLANET SYSTEMS FROM K2 CAMPAIGNS 1 AND 2 AND THE MASSES OF TWO HOT SUPER-EARTHS
}

\author{
Evan Sinukoff ${ }^{1,10}$, Andrew W. Howard ${ }^{1}$, Erik A. Petigura ${ }^{2,11}$, Joshua E. Schlieder ${ }^{3,12}$, Ian J. M. Crossfield ${ }^{4,13}$, \\ David R. Ciardi ${ }^{5}$, Benjamin J. Fulton ${ }^{1,14}$, Howard Isaacson ${ }^{6}$, Kimberly M. Aller ${ }^{1}$, Christoph Baranec ${ }^{1}$, \\ Charles A. Beichman ${ }^{5}$, Brad M. S. Hansen ${ }^{7}$, Heather A. Knutson ${ }^{2}$, Nicholas M. Law ${ }^{8}$, Michael C. Liu ${ }^{1}$, \\ ReEd Riddle ${ }^{9}$, and Courtney D. Dressing ${ }^{2,13}$ \\ ${ }^{1}$ Institute for Astronomy, University of Hawai'i at Mānoa, Honolulu, HI 96822, USA \\ ${ }^{2}$ California Institute of Technology, Pasadena, CA 91125, USA \\ ${ }^{3}$ NASA Ames Research Center, Moffett Field, CA 94035, USA \\ ${ }^{4}$ Lunar \& Planetary Laboratory, University of Arizona, 1629 E. University Blvd., Tucson, AZ 85721, USA \\ ${ }^{5}$ NASA Exoplanet Science Institute, California Institute of Technology, 770 S. Wilson Ave., Pasadena, CA 91125, USA \\ ${ }^{6}$ Astronomy Department, University of California, Berkeley, CA 94720, USA \\ ${ }^{7}$ Department of Physics \& Astronomy and Institute of Geophysics \& Planetary Physics, University of California Los Angeles, Los Angeles, CA 90095, USA \\ 8 Department of Physics and Astronomy, University of North Carolina at Chapel Hill, Chapel Hill, NC 27599, USA \\ ${ }^{9}$ Division of Physics, Mathematics, and Astronomy, California Institute of Technology, Pasadena, CA 91125, USA \\ Received 2015 November 28; revised 2016 May 10; accepted 2016 May 17; published 2016 August 9
}

\begin{abstract}
We present a catalog of 11 multiplanet systems from Campaigns 1 and 2 of the $K 2$ mission. We report the sizes and orbits of 26 planets split between seven two-planet systems and four three-planet systems. These planets stem from a systematic search of the $K 2$ photometry for all dwarf stars observed by $K 2$ in these fields. We precisely characterized the host stars with adaptive optics imaging and analysis of high-resolution optical spectra from Keck/HIRES and medium-resolution spectra from IRTF/SpeX. We confirm two planet candidates by mass detection and validate the remaining 24 candidates to $>99 \%$ confidence. Thirteen planets were previously validated or confirmed by other studies, and 24 were previously identified as planet candidates. The planets are mostly smaller than Neptune $(21 / 26$ planets), as in the Kepler mission, and all have short periods $(P<50$ days $)$ due to the duration of the $K 2$ photometry. The host stars are relatively bright (most have $K p<12.5 \mathrm{mag}$ ) and are amenable to follow-up characterization. For K2-38, we measured precise radial velocities using Keck/HIRES and provide initial estimates of the planet masses. K2-38b is a short-period super-Earth with a radius of $1.55 \pm 0.16 R_{\oplus}$, a mass of $12.0 \pm 2.9 M_{\oplus}$, and a high density consistent with an iron-rich composition. The outer planet K2-38c is a lower-density sub-Neptune-size planet with a radius of $2.42 \pm 0.29 R_{\oplus}$ and a mass of $9.9 \pm 4.6 M_{\oplus}$ that likely has a substantial envelope. This new planet sample demonstrates the capability of $K 2$ to discover numerous planetary systems around bright stars.
\end{abstract}

Key words: planetary systems - stars: late-type - stars: solar-type - techniques: photometric - techniques: radial velocities - techniques: spectroscopic

\section{INTRODUCTION}

The prime Kepler mission (2009-2013) demonstrated that compact, multiplanet systems are common (Howard et al. 2012; Fressin et al. 2013; Petigura et al. 2013). Of the $4000+$ planet candidates from Kepler, roughly 1500 are in systems with multiple candidates (Mullally et al. 2015). Some exceptional systems include the high-multiplicity Kepler-11 (Lissauer et al. 2011a) and Kepler-90 (Schmitt et al. 2014) systems, which host six and seven planets, respectively, all within $1 \mathrm{AU}$. Another noteworthy system is Kepler-36, which hosts two planets with semimajor axes differing by $10 \%$ but densities differing by a factor of eight (Carter et al. 2012).

The ensemble properties of Kepler multiplanet systems ("multis") have provided key insights into the formation, evolution, and architectures of planetary systems (Lissauer et al. 2011b, 2012, 2014; Rowe et al. 2014). Most of the Kepler multis have low $(\lesssim 3 \%)$ mutual inclinations (Fang \&

\footnotetext{
${ }_{11}^{10}$ NSERC Postgraduate Research Fellow.

${ }^{11}$ Hubble Fellow.

12 NASA Postdoctoral Program Fellow.

13 NASA Sagan Fellow.

14 NSF Graduate Research Fellow.
}

Margot 2012). Many multiplanet systems are dynamically packed, i.e., adding an additional planet on an intermediate orbit leads to dynamical instability (Fang \& Margot 2013). While the distribution of orbital period ratios of Kepler multis is roughly uniform, Fabrycky et al. (2014) observed an excess of planet pairs with orbital period ratios exterior to first-order mean motion resonance (MMR) and a deficit of planets lying interior to resonance. This feature may be the outcome of eccentricity damping of resonant planet pairs by the protoplanetary disk (Lithwick \& Wu 2012; Batygin \& Morbidelli 2013).

Systems with multiple transiting planets are particularly valuable because they are a clean sample with nearly zero falsepositive detections (Lissauer et al. 2012, 2014). This is due to the low probability of having multiple stars with a falsepositive signals in the same photometric aperture, i.e., eclipsing binaries (EBs) are distributed sparsely on the sky.

Given the photometric precision and $4 \mathrm{yr}$ baseline of the prime Kepler mission, dynamical interactions between pairs of planets are often detected as transit timing variations (TTVs), which can constrain planet properties such as mass and eccentricity (Agol et al. 2005; Holman \& Murray 2005). The analysis of the Kepler-36 system by Carter et al. (2012) 
Table 1

K2 Multiplanet Host Stars

\begin{tabular}{|c|c|c|c|c|c|c|c|c|c|c|}
\hline$K 2$ Name & EPIC No. & Field & $\begin{array}{c}\text { R.A. } \\
\text { (J2000) }\end{array}$ & $\begin{array}{c}\text { Decl. } \\
(\mathrm{J} 2000)\end{array}$ & $\begin{array}{c}\mu_{\text {R.A. }}{ }^{a} \\
\left(\text { mas yr }^{-1}\right)\end{array}$ & $\begin{array}{c}\mu_{\text {decl. }^{a}}{ }_{\left(\text {mas yr }^{-1}\right)}\end{array}$ & $\begin{array}{c}V^{\mathrm{b}} \\
(\mathrm{mag})\end{array}$ & $\begin{array}{c}K p^{\mathrm{a}} \\
(\mathrm{mag})\end{array}$ & $\begin{array}{c}J^{\mathrm{a}} \\
(\mathrm{mag})\end{array}$ & $\begin{array}{c}K s^{\mathrm{a}} \\
(\mathrm{mag})\end{array}$ \\
\hline $\mathrm{K} 2-5$ & 201338508 & $\mathrm{C} 1$ & $11: 17: 13$ & $-01: 52: 41$ & $-10.3 \pm 4.4$ & $+32.6 \pm 4.6$ & $14.91 \pm 0.03$ & 14.36 & $12.45 \pm 0.03$ & $11.60 \pm 0.02$ \\
\hline $\mathrm{K} 2-3$ & 201367065 & $\mathrm{C} 1$ & $11: 29: 20$ & $-01: 27: 17$ & $+88.3 \pm 2.0$ & $-73.6 \pm 2.7$ & $12.17 \pm 0.01$ & 11.57 & $9.42 \pm 0.03$ & $8.56 \pm 0.02$ \\
\hline $\mathrm{K} 2-8$ & 201445392 & $\mathrm{C} 1$ & 11:19:10 & $-00: 17: 04$ & $-34.7 \pm 4.9$ & $-16.7 \pm 4.1$ & $14.61 \pm 0.03$ & 14.38 & $12.83 \pm 0.03$ & $12.25 \pm 0.03$ \\
\hline K2-19 & 201505350 & $\mathrm{C} 1$ & $11: 39: 50$ & $+00: 36: 13$ & $-18.7 \pm 1.7$ & $+4.5 \pm 2.0$ & $13.00 \pm 0.01$ & 12.81 & $11.60 \pm 0.02$ & $11.16 \pm 0.03$ \\
\hline $\mathrm{K} 2-35$ & 201549860 & $\mathrm{C} 1$ & $11: 20: 25$ & $+01: 17: 09$ & $+10.4 \pm 6.4$ & $-16.8 \pm 5.2$ & $14.35 \pm 0.06$ & 13.92 & $12.14 \pm 0.02$ & $11.42 \pm 0.02$ \\
\hline $\mathrm{K} 2-36$ & 201713348 & $\mathrm{C} 1$ & $11: 17: 48$ & $+03: 51: 59$ & $-17.5 \pm 2.3$ & $+23.5 \pm 2.5$ & $11.80 \pm 0.03$ & 11.53 & $10.03 \pm 0.02$ & $9.45 \pm 0.03$ \\
\hline $\mathrm{K} 2-16$ & 201754305 & $\mathrm{C} 1$ & $11: 40: 23$ & $+04: 33: 26$ & $-3.8 \pm 3.2$ & $+21.8 \pm 3.9$ & $14.67 \pm 0.04$ & 14.30 & $12.76 \pm 0.03$ & $12.09 \pm 0.02$ \\
\hline $\mathrm{K} 2-24$ & 203771098 & $\mathrm{C} 2$ & $16: 10: 18$ & $-24: 59: 25$ & $-60.6 \pm 2.5$ & $-65.4 \pm 2.4$ & $11.07 \pm 0.11$ & 11.65 & $9.64 \pm 0.02$ & $9.18 \pm 0.02$ \\
\hline $\mathrm{K} 2-37$ & 203826436 & $\mathrm{C} 2$ & $16: 13: 48$ & $-24: 47: 13$ & $-9.4 \pm 1.9$ & $+3.8 \pm 2.6$ & $12.52 \pm 0.06$ & 12.24 & $10.69 \pm 0.02$ & $10.14 \pm 0.02$ \\
\hline $\mathrm{K} 2-38$ & 204221263 & $\mathrm{C} 2$ & $16: 00: 08$ & $-23: 11: 21$ & $-55.6 \pm 3.4$ & $-38.3 \pm 3.7$ & $11.39 \pm 0.03$ & 11.21 & $9.91 \pm 0.02$ & $9.47 \pm 0.02$ \\
\hline $\mathrm{K} 2-32$ & 205071984 & $\mathrm{C} 2$ & $16: 49: 42$ & $-19: 32: 34$ & $-16.4 \pm 1.2$ & $-52.5 \pm 1.3$ & $12.31 \pm 0.04$ & 12.01 & $10.40 \pm 0.02$ & $9.82 \pm 0.02$ \\
\hline
\end{tabular}

Notes.

${ }^{\mathrm{a}}$ From Ecliptic Plane Input Catalog (EPIC).

${ }^{\mathrm{b}}$ From AAVSO Photometric All-Sky Survey (APASS) Data Release 9.

demonstrated the power of TTV observations. They measured a mass of $4.45_{-0.27}^{+0.33} M_{\oplus}$ for Kepler-36b, a planet with radius $R_{P}=1.486 \pm 0.035 R_{\oplus}$. Currently, Kepler-36b has the bestconstrained mass of any exoplanet smaller than $2 R_{\oplus}$ (Dressing et al. 2015).

The prime Kepler mission came to an end in 2013, following the failure of a second reaction wheel. Beginning in 2014 March, NASA began operating the telescope in a new mode called $K 2$ (Howell et al. 2014). During $K 2$ operations, the spacecraft observes a different region of the ecliptic plane every $\sim 85$ days.

Kepler planet catalogs (Borucki et al. 2011; Batalha et al. 2013; Burke et al. 2014; Mullally et al. 2015; Rowe et al. 2015) spawned numerous statistical studies on planet occurrence, the distribution of planet sizes, and the diversity of system architectures. These studies deepened our understanding of planet formation and evolution. Continuing in this pursuit, $K 2$ planet catalogs will provide a wealth of planets around bright stars that are particularly favorable for studying planet compositions - perhaps the best link to their formation histories.

The first four $K 2$ campaigns ( $\mathrm{C} 0-\mathrm{C} 3)$ plus an additional engineering test campaign have yielded over 230 planet candidates at the time of writing. ${ }^{15}$ Moreover, $\sim 40$ of these planet candidates have been either statistically validated as planets at better than $99 \%$ confidence or confirmed via radial velocity (RV) or TTV detection, including several noteworthy discoveries. Super-Earth HIP 116454 b, discovered in the $K 2$ engineering test field, orbits a bright $\mathrm{K}$ dwarf. Its mass is well constrained from follow-up RV measurements (Vanderburg et al. 2015). From C1, Crossfield et al. (2015) announced three super-Earths orbiting a nearby M0 dwarf, K2-3. Almenara et al. (2015) and Dai et al. (2016) detected the RV signature of the inner planet, which is consistent with a mostly rocky composition, although the water fraction could be as large as $60 \%$. Foreman-Mackey et al. (2015) discovered two planets of Neptune and Saturn size near a 3:2 MMR around K2-19. Armstrong et al. (2015) used TTVs to constrain the mass of the larger outer planet, and the masses of both planets were measured by Dai et al. (2016) using RVs. Vanderburg et al. (2016) reported a third Earth-size planet candidate, K2-19d, at

\footnotetext{
15 NASA Exoplanet Archive, UT 2016 April 28, http://exoplanetarchive. ipac.caltech.edu.
}

$P=2.5$ days. The first $K 2$ planet catalogs have already been assembled; Foreman-Mackey et al. (2015) reported 36 planet candidates, 21 of which were validated at $>99 \%$ confidence by Montet et al. (2015b). These include four validated multiplanet systems (K2-3, K2-5, K2-16, K2-19) and one system, K2-8, with one validated planet and a second planet candidate. More recently, Vanderburg et al. (2016) presented 234 planet candidates in $\mathrm{C} 0-\mathrm{C} 3$, including 20 systems with multiple planet candidates.

In this paper, we present 11 multiplanet systems with a total of 26 planets detected by our team in $K 2$ photometry from Campaigns 1 and 2 (C1 and $\mathrm{C} 2$ ). We detected no multiplanet systems in Campaign 0. We adopt a "confirmed" disposition for planet candidates with detected RV or TTV signatures and, following Montet et al. (2015b), a "validated" disposition for planet candidates found to have a false-positive probability (FPP) of $<1 \%$. Under this definition, 13 of the 26 planet candidates are previously confirmed or validated, 11 are newly validated, and 2 are newly discovered and confirmed. Most importantly, 9 of the 13 newly validated or confirmed planet candidates orbit stars with $V \leqslant 12.5 \mathrm{mag}$, amenable to $\mathrm{RV}$ follow-up. For one system, K2-38, we obtained RV measurements using Keck/HIRES to constrain planet masses. The remainder of this paper is organized as follows: In Section 2 we describe the photometric detection of multis in $K 2$ photometry along with follow-up observations that confirm the planets and characterize their stellar hosts. Section 3 details the physical properties of stellar hosts. Section 4 outlines our validation of each planet via adaptive optics (AO) images, archival images, and vetting of the light curves and spectra. In Section 5, we describe our light-curve modeling and present derived planet properties. We present our RV measurements of K2-38 and the derived planet masses in Section 6. In Section 7, we summarize the most noteworthy characteristics of each system, including additional findings of other studies where relevant. We discuss the likely compositions of the K2-38 planets, summarize the ensemble properties of our planet sample, and compare our results to other studies in Section 8. Our RVs, spectra, AO images, and contrast curves will be uploaded to the ExoFOPK2 website. ${ }^{16}$ We note that throughout this paper, systems are ordered by EPIC number.

\footnotetext{
${ }^{16}$ https://cfop.ipac.caltech.edu/k2/
} 


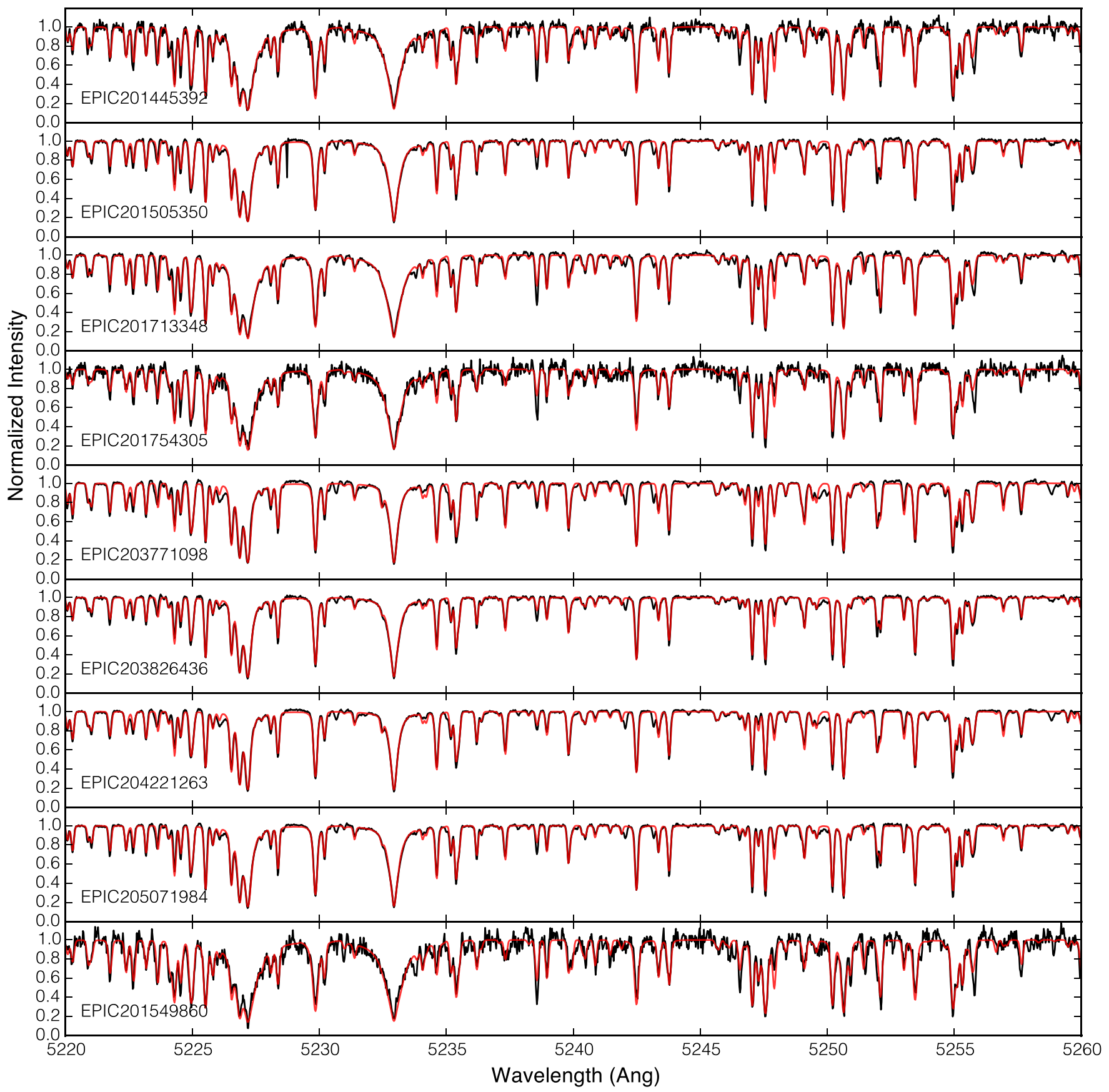

Figure 1. Representative segment of our HIRES spectra spanning $\lambda=5220-5260 \AA$ A. The observed stellar spectra are shown in black, and the best-fit SpecMatch models (Petigura 2015) are overplotted in red. Note that this represents only about $10 \%$ of the wavelength coverage modeled by SpecMatch.

\section{DETECTION AND OBSERVATIONS OF K2 MULTIS}

\subsection{K2 Planet Search Program}

During the prime Kepler mission, the project office selected nearly all of the stars that were observed. This target list was dominated by a magnitude-limited set of F, G, K, and M dwarfs $(K p<16)$, from which the major planet catalogs and occurrence analyses were derived. $K 2$ is entirely community driven, with all targets selected from Guest Observer proposals. Our team has proposed large samples of G, K, and M dwarfs for every $K 2$ Campaign (to date, Campaigns $0-10$ ). For the $\mathrm{G}$ and $\mathrm{K}$ dwarfs, our proposed sets of stars are magnitude limited at $K p<13$ or 14 (depending on crowding) and $\sim 3500-10,000$ stars per Campaign have been selected for $K 2$ observations. During each $K 2$ Campaign, the Kepler telescope observes the selected stars nearly continuously for $\sim 75$ days.

This catalog of multiplanet systems is based on photometry collected by $K 2$ during Campaign 1 (2014 May 30-August 21) and Campaign 2 (2014 August 23-November 13). The stars were part of $K 2$ Guest Observer proposals led by I. Crossfield, R. Sanchis-Ojeda, A. Scholz, A. Sozzetti, P. Robertson, D. Stello, V. Sanchez Bejar, N. Deacon, and B.-O. Demory (Campaign 1) and by E. Petigura, R. Sanchis-Ojeda, and D. Stello (Campaign 2). We searched for transiting planets in the photometry of all stars observed by $K 2$, not just those in the above programs. 
Table 2

Spectroscopic Stellar Properties

\begin{tabular}{|c|c|c|c|c|c|c|c|c|}
\hline$K 2$ Name & EPIC Number & $\begin{array}{l}T_{\text {eff }} \\
(\mathrm{K})\end{array}$ & $\begin{array}{l}\log g \\
(\mathrm{cgs})\end{array}$ & $\begin{array}{c}{[\mathrm{Fe} / \mathrm{H}]} \\
(\mathrm{dex})\end{array}$ & $\underset{\left(\mathrm{km} \mathrm{s}^{-1}\right)}{v \sin i}$ & $\begin{array}{c}\log R_{\mathrm{HK}}^{\prime} \\
(\mathrm{dex})\end{array}$ & $\begin{array}{c}M_{\star} \\
\left(M_{\odot}\right)\end{array}$ & $\begin{array}{c}R_{\star} \\
\left(R_{\odot}\right)\end{array}$ \\
\hline K2-5 & 201338508 & $3930 \pm 375$ & $4.71 \pm 0.21$ & $-0.33 \pm 0.19$ & $\ldots$ & $\ldots$ & $0.61 \pm 0.13$ & $0.57 \pm 0.12$ \\
\hline $\mathrm{K} 2-3$ & 201367065 & $3896 \pm 189$ & $4.72 \pm 0.13$ & $-0.32 \pm 0.13$ & $\ldots$ & $\ldots$ & $0.60 \pm 0.09$ & $0.56 \pm 0.07$ \\
\hline K2-8 & 201445392 & $4870 \pm 60$ & $4.52 \pm 0.10$ & $-0.02 \pm 0.04$ & $<2$ & $\ldots$ & $0.78 \pm 0.04$ & $0.74 \pm 0.04$ \\
\hline K2-19 & 201505350 & $5430 \pm 60$ & $4.63 \pm 0.10$ & $+0.10 \pm 0.04$ & $<2$ & -4.66 & $0.93 \pm 0.05$ & $0.86 \pm 0.04$ \\
\hline $\mathrm{K} 2-35$ & 201549860 & $4680 \pm 60$ & $4.56 \pm 0.10$ & $+0.04 \pm 0.04$ & $3 \pm 1$ & $\ldots$ & $0.76 \pm 0.04$ & $0.72 \pm 0.04$ \\
\hline $\mathrm{K} 2-36$ & 201713348 & $4924 \pm 60$ & $4.65 \pm 0.10$ & $-0.03 \pm 0.04$ & $2 \pm 1$ & $\ldots$ & $0.80 \pm 0.04$ & $0.74 \pm 0.04$ \\
\hline $\mathrm{K} 2-16$ & 201754305 & $4742 \pm 60$ & $4.51 \pm 0.10$ & $-0.33 \pm 0.04$ & $2 \pm 1$ & $\ldots$ & $0.68 \pm 0.03$ & $0.66 \pm 0.03$ \\
\hline K2-24 & 203771098 & $5743 \pm 60$ & $4.29 \pm 0.08$ & $+0.42 \pm 0.04$ & $<2$ & -5.15 & $1.12 \pm 0.06$ & $1.21 \pm 0.12$ \\
\hline $\mathrm{K} 2-37$ & 203826436 & $5413 \pm 60$ & $4.52 \pm 0.10$ & $-0.03 \pm 0.04$ & $<2$ & -4.85 & $0.90 \pm 0.05$ & $0.85 \pm 0.04$ \\
\hline $\mathrm{K} 2-38$ & 204221263 & $5757 \pm 60$ & $4.35 \pm 0.08$ & $+0.28 \pm 0.04$ & $<2$ & -5.13 & $1.07 \pm 0.05$ & $1.10 \pm 0.09$ \\
\hline $\mathrm{K} 2-32$ & 205071984 & $5315 \pm 60$ & $4.43 \pm 0.10$ & $+0.00 \pm 0.04$ & $<2$ & -4.94 & $0.87 \pm 0.04$ & $0.87 \pm 0.05$ \\
\hline
\end{tabular}

Notes.

For K2-5 and K2-3, $T_{\text {eff }},[\mathrm{Fe} / \mathrm{H}], M_{\star}$, and $R_{\star}$ are derived using spectroscopic indices of Mann et al. (2013a) and empirical relations of Boyajian et al. (2012). For the other stars $T_{\mathrm{eff}}, \log g,[\mathrm{Fe} / \mathrm{H}]$, and $v \sin i$ are derived using SpecMatch (Petigura 2015), $\log R_{\mathrm{HK}}^{\prime}$ is derived using the recipe of Isaacson \& Fischer (2010), and $M_{\star}$ and $R_{\star}$ are derived using the isochrones Python package (Morton 2015a). We only list $\log R_{\mathrm{HK}}^{\prime}$ for stars with $T_{\text {eff }}>5000 \mathrm{~K}$, for which this activity metric is well calibrated. $S_{\mathrm{HK}}$ values for cooler stars K2-8, K2-35, K2-36, and K2-16 are 0.33, 0.33, 0.46, and 0.18, respectively. Spectra of K2-5 and K2-3 come from observations with IRTF/SpeX and do not contain $\mathrm{Ca}$ II $\mathrm{H}$ and $\mathrm{K}$ lines.

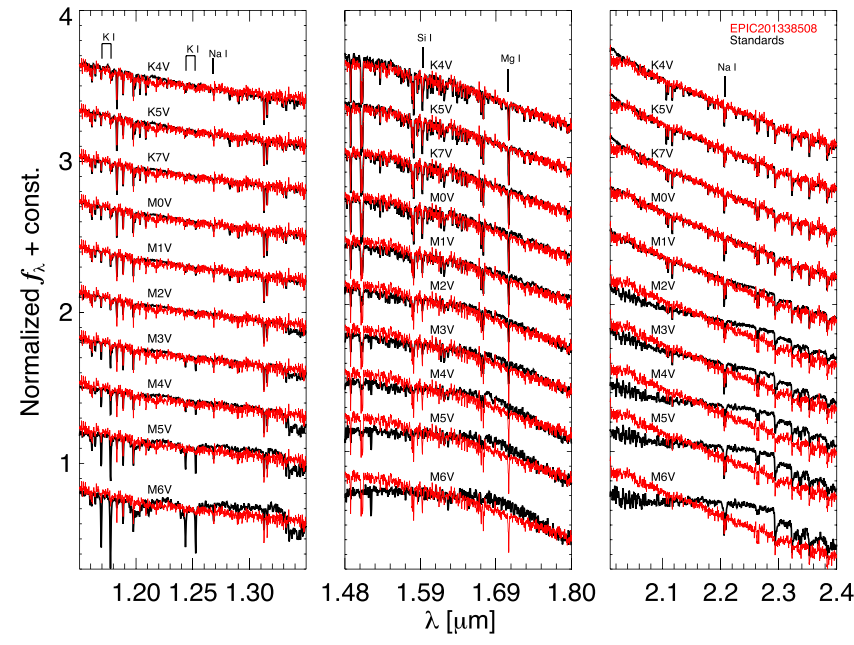

Figure 2. $J H K$-band IRTF/SpeX spectra of K2-5 compared to late-type standards from the IRTF spectral library. All spectra are normalized to the continuum in each of the plotted regions. The star is a best visual match to spectral type $\sim \mathrm{K} 7$ across the three near-IR bands. This is consistent with the results from our analyses using spectroscopic indices.

\subsection{K2 Photometry and Transit Detection}

During $K 2$ observations, solar radiation pressure exerts a torque on the spacecraft that causes the telescope to roll around its boresight. Consequently, stars trace out small arcs of $\sim 1$ pixel every $\sim 6 \mathrm{hr}$. As the stars sample different pixel phases, interpixel sensitivity variations cause their apparent brightnesses to change. Disentangling stellar variability from spacecraft systematics is nontrivial when working with $K 2$ data.

We extracted the photometry from the $K 2$ target pixel files, which are available at the Mikulski Archive for Space Telescopes (MAST). ${ }^{17}$ Our photometric extraction procedure is detailed in Crossfield et al. (2015). In brief, for a given target star we compute raw aperture photometry using a soft-edged circular aperture. For every frame in a $K 2$ campaign, we solve for the roll angle between the target and an arbitrary reference

\footnotetext{
${ }^{17}$ https://archive.stsci.edu/k2/
}

frame using several hundred stars. We model the time- and rolldependent variations using a Gaussian process, which are then subtracted from the raw photometry to produce calibrated photometry. This process is repeated for different aperture sizes, and we adopt the aperture size that minimizes photometric noise on $3 \mathrm{hr}$ timescales. Specifically, we use the median absolute deviation (MAD) of the $3 \mathrm{hr}$ Single Event Statistic (SES) as our noise metric. We define the SES as the depth of a box-shaped dimming relative to the local photometric level. Conceptually, this is similar to the $3 \mathrm{hr}$ Combined Differential Photometric Precision (CDPP-3) metric used by the Kepler project. We compute a $3 \mathrm{hr}$ SES at every long-cadence measurement as part of our transit search. ${ }^{18}$ This method of aperture selection favors small apertures for faint stars (where background noise dominates) and large apertures for bright targets.

To search the calibrated photometry for planetary transits, we use the TERRA algorithm (Petigura et al. 2013). We have adapted TERRA to search for multiplanet systems. When TERRA identifies a candidate transit, it flags that star for additional analysis. TERRA masks out the transit of the first candidate along with a buffer of $\Delta T$ on either side, where $\Delta T$ is the transit duration. TERRA then repeats the transit search in the masked photometry. This process continues until no transits with signal-to-noise ratio $(\mathrm{S} / \mathrm{N})>8$ are detected or when the number of iterations exceeds 5 .

Table 1 lists coordinates, proper motions, and multiband photometry for the 11 stars around which we detect multiple transiting planets.

\subsection{AO Imaging}

We obtained near-infrared AO images of the 11 EPIC sources at the W. M. Keck Observatory on the nights of 2015 April 1 UT (K2-8, K2-36, K2-19, K2-35, K2-24, K2-37, K232), 2015 April 7 UT (K2-3, K2-5), and 25 July 2015

\footnotetext{
18 As an example, to compute the SES on $1 \mathrm{hr}$ timescales (corresponding to two Kepler long-cadence measurements), we construct the following kernel: $g=\frac{1}{2}[0.5,0.5,-1,-1,0.5,0.5]$, which is convolved with the measured photometry. See Petigura \& Marcy (2012) for further details.
} 

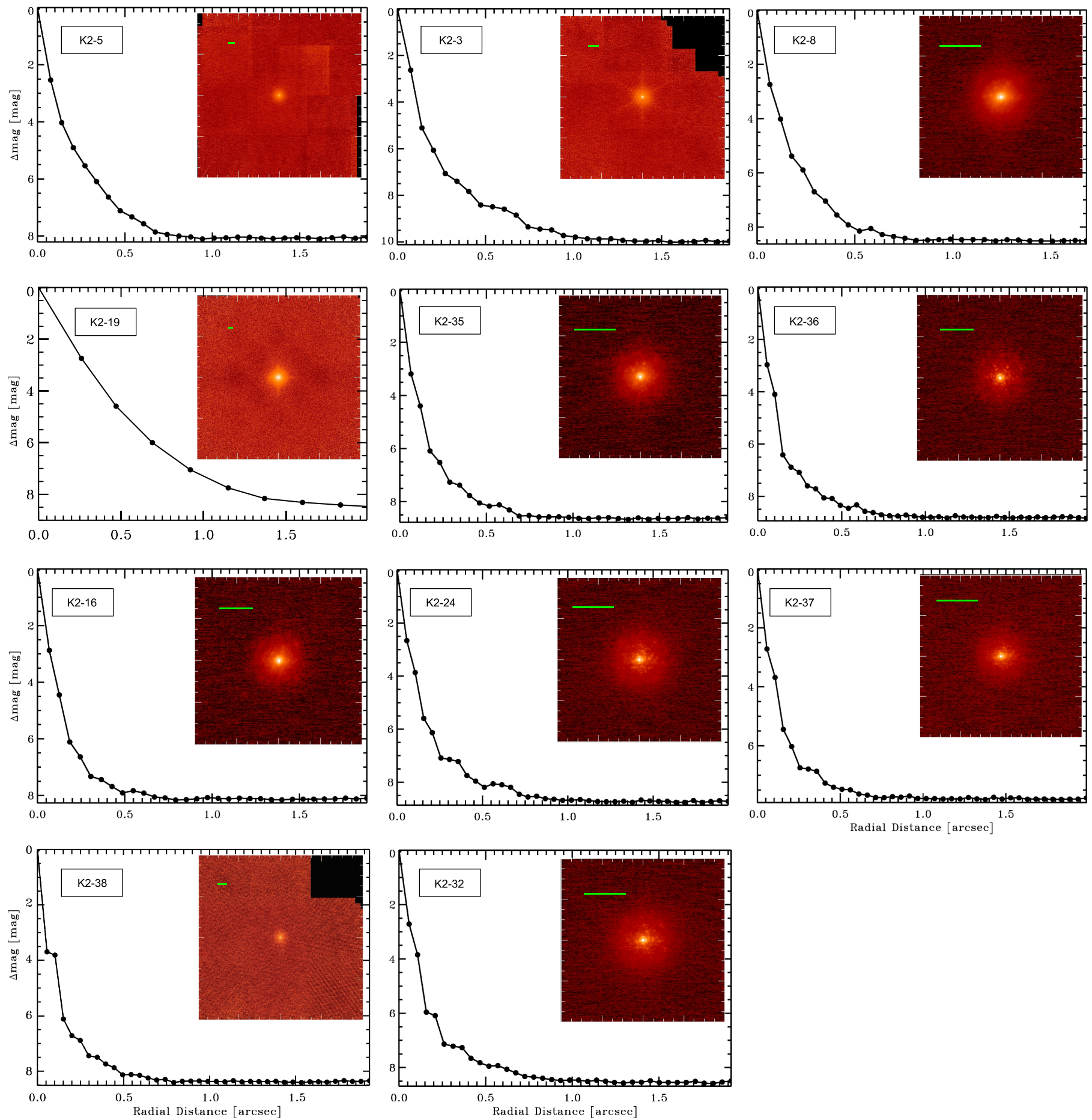

Figure 3. AO images and contrast curves for all multiplanet hosts. Targets were imaged using Keck/NIRC2 AO, with the exception of K2-19, which was observed with Palomar/PHARO. Green horizontal lines correspond to $1^{\prime \prime}$ in each field. Dotted black lines indicate where companions would be detectable with $5 \sigma$ confidence. No companions were detected near any of the 11 stars.

UT (K2-38) and at Palomar Observatory on the night of 2015 May 29 UT (K2-19). The observations were obtained with the $1024 \times 1024$ NIRC2 array at Keck Observatory behind the natural guide star AO system and the $1024 \times 1024$ PHARO array behind the PALM-3000 natural guide star system (Dekany et al. 2013). In all cases, the target star was bright enough to be used as the guide star. NIRC2 has a pixel scale of 9.942 mas pixel $^{-1}$ with a field of view of $10^{\prime \prime}$; PHARO has a pixel scale of 25 mas pixel ${ }^{-1}$ with a field of view of 25"'6. The observations were taken in either the Ks or Br $\gamma$ filters; $\operatorname{Br} \gamma$ has a narrower bandwidth $(2.13-2.18 \mu \mathrm{m})$ but a similar central wavelength $(2.15 \mu \mathrm{m})$ compared to the $K s$ filter $(1.95-2.34 \mu \mathrm{m})$ and allows for longer integration times before saturation. For the Keck observations, a three-point dither pattern was utilized to avoid the noisier lower left quadrant of the NIRC2 array; the three-point dither pattern was observed three times, for a total 
Table 3

Summary of $K 2$ Multiplanet Systems

\begin{tabular}{|c|c|c|c|c|c|c|c|c|c|}
\hline $\begin{array}{l}K 2 \\
\text { Name }\end{array}$ & EPIC No. & $\begin{array}{l}T_{\text {eff }} \\
(\mathrm{K})\end{array}$ & $\begin{array}{c}K p \\
(\mathrm{mag})\end{array}$ & $\begin{array}{c}T_{0} \\
\left(\mathrm{BJD}_{\mathrm{TDB}}-2,456,000\right)\end{array}$ & $\begin{array}{c}P \\
\text { (days) }\end{array}$ & $\begin{array}{c}R_{p} \\
\left(R_{\oplus}\right)\end{array}$ & $\begin{array}{l}T_{\mathrm{eq}} \\
(\mathrm{K})\end{array}$ & FPP & $\begin{array}{l}\text { Dispo- } \\
\text { sition }^{\text {a }}\end{array}$ \\
\hline K2-5 & 201338508 & $3930 \pm 375$ & 14.36 & & & & & & \\
\hline $\mathrm{K} 2-5 \mathrm{~b}$ & $201338508 b$ & & & $808.8600 \pm 0.0048$ & $5.73594 \pm 0.00064$ & $1.91 \pm 0.44$ & $565 \pm 84$ & $<0.001$ & Valid. \\
\hline $\mathrm{K} 2-5 \mathrm{c}$ & $201338508 \mathrm{c}$ & & & $814.6010 \pm 0.0052$ & $10.93241 \pm 0.00134$ & $2.26 \pm 0.62$ & $456 \pm 68$ & $<0.001$ & Valid. \\
\hline $\mathrm{K} 2-3$ & 201367065 & $3896 \pm 189$ & 11.57 & & & & & & \\
\hline $\mathrm{K} 2-3 \mathrm{~b}$ & $201367065 b$ & & & $813.4173 \pm 0.0011$ & $10.05449 \pm 0.00026$ & $2.18 \pm 0.30$ & $463 \pm 39$ & $<0.001$ & Conf. \\
\hline $\mathrm{K} 2-3 \mathrm{c}$ & $201367065 \mathrm{c}$ & & & $812.2812 \pm 0.0022$ & $24.64354 \pm 0.00117$ & $1.85 \pm 0.27$ & $344 \pm 29$ & $<0.001$ & Valid. \\
\hline $\mathrm{K} 2-3 \mathrm{~d}$ & $201367065 d$ & & & $826.2288 \pm 0.0034$ & $44.55983 \pm 0.00590$ & $1.51 \pm 0.23$ & $282 \pm 24$ & $<0.001$ & Valid. \\
\hline $\mathrm{K} 2-8$ & 201445392 & $4870 \pm 60$ & 14.38 & & & & & & \\
\hline $\mathrm{K} 2-8 \mathrm{~b}$ & $201445392 b$ & & & $813.6114 \pm 0.0030$ & $10.35239 \pm 0.00086$ & $3.58 \pm 0.71$ & $631 \pm 18$ & 0.013 & Valid. $^{\mathrm{b}}$ \\
\hline $\mathrm{K} 2-8 \mathrm{c}$ & $201445392 \mathrm{c}$ & & & $813.0707 \pm 0.0033$ & $5.06416 \pm 0.00041$ & $2.41 \pm 0.33$ & $801 \pm 23$ & 0.008 & Valid. \\
\hline K2-19 & 201505350 & $5430 \pm 60$ & 12.81 & & & & & & \\
\hline $\mathrm{K} 2-19 \mathrm{~b}$ & $201505350 \mathrm{~b}$ & & & $813.3837 \pm 0.0003$ & $7.91940 \pm 0.00005$ & $7.74 \pm 0.39$ & $854 \pm 24$ & $<0.001$ & Conf. \\
\hline $\mathrm{K} 2-19 \mathrm{c}$ & $201505350 \mathrm{c}$ & & & $817.2755 \pm 0.0051$ & $11.90715 \pm 0.00150$ & $4.86_{-0.44}^{+0.62}$ & $745 \pm 21$ & $<0.001$ & Conf. \\
\hline K2-19d & $201505350 \mathrm{~d}$ & & & $808.9207 \pm 0.0086$ & $2.50856 \pm 0.00041$ & $1.14 \pm 0.13$ & $1252 \pm 36$ & $<0.001$ & Valid. \\
\hline $\mathrm{K} 2-35$ & 201549860 & $4680 \pm 60$ & 13.92 & & & & & & \\
\hline $\mathrm{K} 2-35 \mathrm{~b}$ & $201549860 \mathrm{~b}$ & & & $810.5871 \pm 0.0085$ & $2.39984 \pm 0.00039$ & $1.40 \pm 0.17$ & $979 \pm 29$ & $<0.001$ & Valid. \\
\hline $\mathrm{K} 2-35 \mathrm{c}$ & $201549860 \mathrm{c}$ & & & $812.1158 \pm 0.0049$ & $5.60912 \pm 0.00071$ & $2.09_{-0.24}^{+0.33}$ & $737 \pm 22$ & $<0.001$ & Valid. \\
\hline $\mathrm{K} 2-36$ & 201713348 & $4924 \pm 60$ & 11.53 & & & & & & \\
\hline $\mathrm{K} 2-36 \mathrm{~b}$ & $201713348 b$ & & & $809.4684 \pm 0.0017$ & $1.42266 \pm 0.00005$ & $1.32 \pm 0.09$ & $1232 \pm 36$ & $<0.001$ & Valid. \\
\hline $\mathrm{K} 2-36 \mathrm{c}$ & $201713348 \mathrm{c}$ & & & $812.8422 \pm 0.0008$ & $5.34059 \pm 0.00010$ & $2.80_{-0.31}^{+0.43}$ & $793 \pm 23$ & $<0.001$ & Valid. \\
\hline K2-16 & 201754305 & $4742 \pm 60$ & 14.30 & & & & & & \\
\hline $\mathrm{K} 2-16 \mathrm{~b}$ & $201754305 b$ & & & $811.6871 \pm 0.0038$ & $7.61880 \pm 0.00087$ & $2.02 \pm 0.24$ & $658 \pm 19$ & $<0.001$ & Valid. \\
\hline $\mathrm{K} 2-16 \mathrm{c}$ & $201754305 \mathrm{c}$ & & & $809.4800 \pm 0.0091$ & $19.07863 \pm 0.00327$ & $2.54_{-0.47}^{+1.12}$ & $485 \pm 14$ & 0.002 & Valid. \\
\hline K2-24 & 203771098 & $5743 \pm 60$ & 11.65 & & & & & & \\
\hline $\mathrm{K} 2-24 \mathrm{~b}$ & $203771098 b$ & & & $905.7950 \pm 0.0007$ & $20.88508 \pm 0.00036$ & $5.83 \pm 0.60$ & $709 \pm 36$ & $<0.001$ & Conf. \\
\hline $\mathrm{K} 2-24 \mathrm{c}$ & $203771098 \mathrm{c}$ & & & $915.6250 \pm 0.0005$ & $42.36342 \pm 0.00063$ & $8.10 \pm 0.82$ & $560 \pm 29$ & $<0.001$ & Conf. \\
\hline K2-37 & 203826436 & $5413 \pm 60$ & 12.24 & & & & & & \\
\hline $\mathrm{K} 2-37 \mathrm{~b}$ & $203826436 b$ & & & $893.7013 \pm 0.0080$ & $4.44117 \pm 0.00075$ & $1.61 \pm 0.17$ & $974 \pm 32$ & 0.009 & Valid. \\
\hline $\mathrm{K} 2-37 \mathrm{c}$ & $203826436 c$ & & & $898.8603 \pm 0.0023$ & $6.42904 \pm 0.00036$ & $2.75 \pm 0.27$ & $861 \pm 28$ & $<0.001$ & Valid. \\
\hline $\mathrm{K} 2-37 \mathrm{~d}$ & $203826436 d$ & & & $907.2315 \pm 0.0031$ & $14.09189 \pm 0.00135$ & $2.73 \pm 0.36$ & $663 \pm 22$ & $<0.001$ & Valid. \\
\hline $\mathrm{K} 2-38$ & 204221263 & $5757 \pm 60$ & 11.21 & & & & & & \\
\hline $\mathrm{K} 2-38 \mathrm{~b}$ & $204221263 b$ & & & $896.8786 \pm 0.0054$ & $4.01593 \pm 0.00050$ & $1.55 \pm 0.16$ & $1184 \pm 51$ & $<0.001$ & Conf. \\
\hline $\mathrm{K} 2-38 \mathrm{c}$ & $204221263 \mathrm{c}$ & & & $900.4752 \pm 0.0033$ & $10.56103 \pm 0.00090$ & $2.42 \pm 0.29$ & $858 \pm 37$ & $<0.001$ & Conf. \\
\hline K2-32 & 205071984 & $5315 \pm 60$ & 12.01 & & & & & & \\
\hline $\mathrm{K} 2-32 \mathrm{~b}$ & $205071984 b$ & & & $900.9258 \pm 0.0009$ & $8.99218 \pm 0.00020$ & $5.38 \pm 0.35$ & $769 \pm 25$ & $<0.001$ & Conf. \\
\hline $\mathrm{K} 2-32 \mathrm{c}$ & $205071984 \mathrm{c}$ & & & $899.4306 \pm 0.0101$ & $20.65614 \pm 0.00598$ & $3.48_{-0.42}^{+0.97}$ & $583 \pm 19$ & 0.022 & Valid. $^{\mathrm{b}}$ \\
\hline K2-32d & $205071984 d$ & & & $903.7846 \pm 0.0031$ & $31.71922 \pm 0.00236$ & $3.75 \pm 0.40$ & $505 \pm 16$ & $<0.001$ & Valid. \\
\hline
\end{tabular}

Notes. $T_{0}=$ mid-transit time; $T_{\mathrm{eq}}=$ equilibrium temperature assuming albedo $=0.3$.

${ }^{\mathrm{a}}$ Conf. = planet candidate confirmed by RV and/or TTV detections. Cand. = planet candidate (FPP $\left.>1 \%\right)$. Valid. = Statistically validated planet candidate, $>99 \%$ confidence (FPP $<1 \%$ ). Dispositions take into account this study and other previously published studies.

b Although the FPPs of K2-8b and K2-32c exceed our 1\% threshold for a "validated" disposition, FPP values do not account for the "multiplicity boost" (reduction in FPP) resulting from the presence of additional planet candidates around the same star. We estimate that the multiplicity boosts for C1 and C2 are large enough by an order of magnitude to validate K2-8b and K2-32c to better than 99\% confidence (See Section 4.4).

of nine frames. The Palomar observations were obtained with a five-point dither pattern with three observations at each dither pattern position, for a total of 15 frames.

To optimize our use of NIRC2 and PHARO, we prescreened three of the targets by acquiring visible-light AO images of $\mathrm{K} 2$ 3, K2-19, and K2-36 on 2015 March 8-9 using the Robo-AO system (Baranec et al. 2013, 2014) on the $1.5 \mathrm{~m}$ Telescope at Palomar Observatory. Observations comprise a sequence of full-frame-transfer EMCCD detector readouts at the maximum rate of $8.6 \mathrm{~Hz}$ for a total of $120 \mathrm{~s}$ of integration time with a long-pass filter cutting on at $600 \mathrm{~nm}$, with longer-wavelength sensitivity limited by the quantum efficiency of the silicon detector out to $1000 \mathrm{~nm}$. The individual $44^{\prime \prime} \times 44^{\prime \prime}$ images are corrected for detector bias and flat-fielding effects before being combined using post-facto shift-and-add processing using the source as the tip-tilt star with $100 \%$ frame selection to synthesize a long-exposure image (Law et al. 2014). Sensitivity to faint stellar companions matched that of the highperformance detectable magnitude ratio in Law et al. (2014), typically $\Delta$ mag $=5$ at $5 \sigma$ at 0 ". 5 . For these three sources, no stellar companions were detected.

\subsection{Spectroscopy \\ 2.4.1. Keck/HIRES}

We used HIRES (Vogt et al. 1994) at the W. M. Keck Observatory to measure high-resolution optical spectra of all 11 stars except for the coolest and faintest star, K2-5. Our observations followed standard procedures of the California 


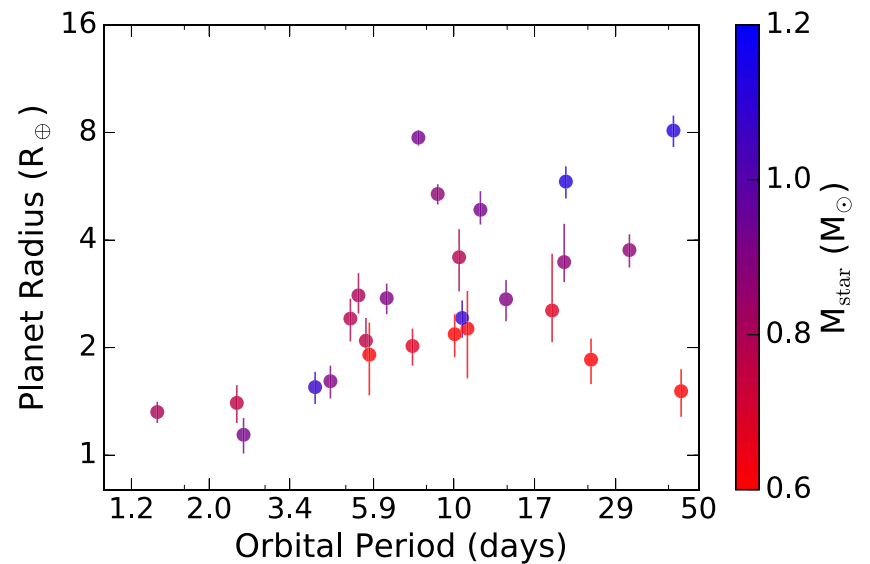

Figure 4. Radii and orbital periods of all 26 planets detected in 11 multiplanet systems in $K 2$ Campaigns 1 and 2. The points are colored according to host star mass, with redder colors corresponding to less massive stars. Twenty-one of the 26 planets are likely smaller than $4 R_{\oplus}$.

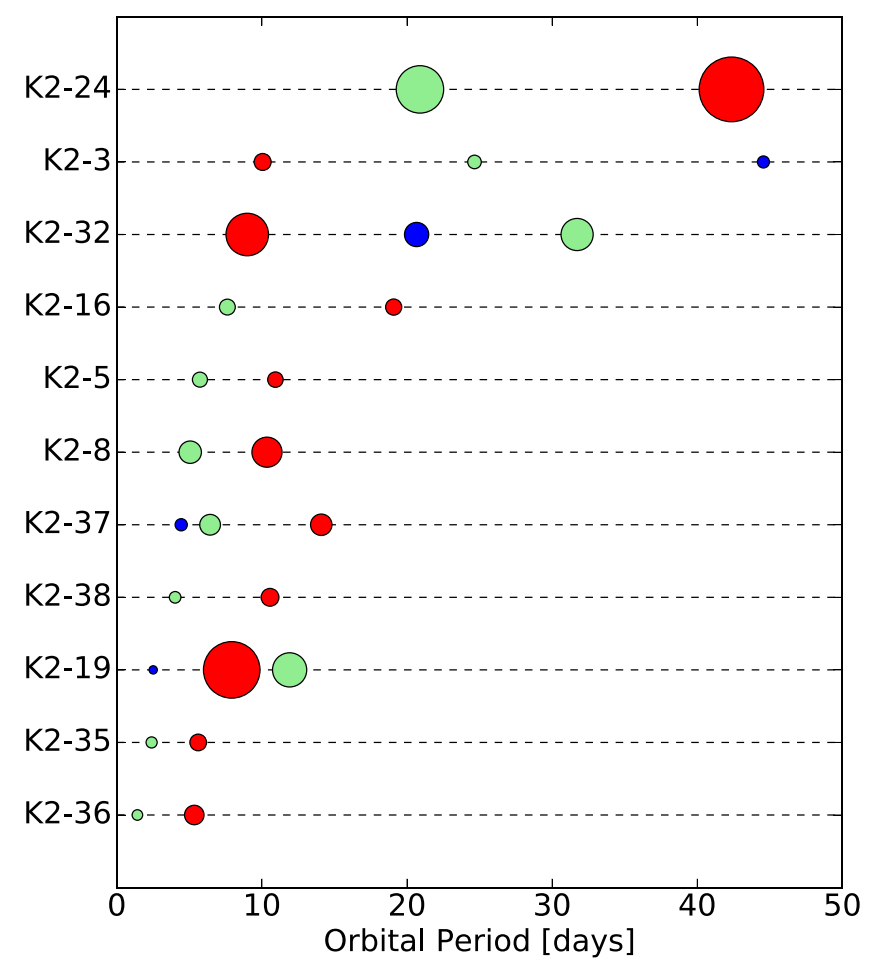

Figure 5. Architecture of the $11 K 2$ in this study. Each row shows the planets in a particular system ordered top to bottom by decreasing orbital period of the inner planet. The symbol sizes are proportional to planet sizes. The largest planet in each system is colored red, the second-largest planet is green, and the third-largest planet (if present) is blue.

Planet Search (CPS; Howard et al. 2010). We used the "C2" decker $\left(0\right.$ " $87 \times 14^{\prime \prime}$ slit) for a spectral resolution $R=55,000$ and subtracted the faint sky spectrum from the stellar spectrum. The HIRES exposure meter was set to achieve the desired S/N, which varied with stellar brightness. For our $K 2$ follow-up program we generally obtain spectra of stars $V \leqslant 13.0 \mathrm{mag}$ having $\mathrm{S} / \mathrm{N}=45 \mathrm{pixel}^{-1}$ at $550 \mathrm{~nm}$, while spectra of fainter stars (K2-8, K2-35) have $\mathrm{S} / \mathrm{N}=32 \mathrm{pixel}^{-1}$. These exposure levels were chosen to be sufficient for determination of stellar parameters while keeping exposure times relatively short (1-10 minutes). Figure 1 shows a wavelength segment of our
HIRES spectra. Some of these spectra have higher $\mathrm{S} / \mathrm{N}$ than prescribed because we obtained additional HIRES spectra for potential Doppler campaigns.

\subsubsection{IRTF/SpeX}

For two $K 2$ multiplanet candidates with near-IR spectral types consistent with $\mathrm{M}$ dwarfs $\left(J-K_{s} \gtrsim 0.8\right)$, we obtained spectra using the near-infrared cross-dispersed spectrograph SpeX (Rayner et al. 2003) on the $3.0 \mathrm{~m}$ NASA Infrared Telescope Facility (IRTF). These stars are K2-3 and K2-5. Our SpeX observations and analyses of K2-3 are described in detail in Crossfield et al. (2015), and we adopt those results here.

We observed K2-5 on 2015 May 5 UT under clear skies with an average seeing of 0.15 . We used SpeX in short crossdispersed mode using the $0.13 \times 15^{\prime \prime}$ slit, which provides wavelength coverage from 0.68 to $2.5 \mu \mathrm{m}$ at a resolution of $R$ $\approx 2000$. The star was dithered to two positions along the slit following an ABBA pattern for sky subtraction. The K2-5 observing sequence consisted of $8 \times 75 \mathrm{~s}$ exposures for a total integration time of $600 \mathrm{~s}$. We also observed an A0 standard and flat and arc lamp exposures immediately after the target star for telluric correction and wavelength calibration.

The data were reduced using the SpeXTool package (Vacca et al. 2003; Cushing et al. 2004). SpeXTool performs flatfielding, bad pixel removal, wavelength calibration, sky subtraction, spectral extraction and combination, telluric correction, flux calibration, and order merging. The final calibrated K2-5 spectrum had $J H K$-band $\mathrm{S} / \mathrm{N} \sim 50 / 75 / 60$. The spectrum is compared to late-type standards from the IRTF Spectral Library ${ }^{19}$ (Cushing et al. 2005; Rayner et al. 2009) in Figure 2.

\section{HOST STAR CHARACTERIZATION}

We used SpecMatch (Petigura 2015) to determine stellar properties from our HIRES spectra for nine stars with spectral types of $\sim \mathrm{K} 4$ and earlier. SpecMatch estimates effective temperatures, surface gravities, metalicities, and rotational velocities by matching HIRES spectra to an interpolated library of model spectra from Coelho et al. (2005). These models are in good agreement with the spectra of wellcharacterized stars for $T_{\text {eff }}>4700 \mathrm{~K}$. See Petigura (2015) for details on SpecMatch, including demonstration that the uncertainties on $T_{\text {eff }}, \log g$, and $[\mathrm{Fe} / \mathrm{H}]$ are $60 \mathrm{~K}$, $0.08-0.10 \mathrm{dex}$, and $0.04 \mathrm{dex}$, respectively. Figure 1 shows the best-fit SpecMatch model spectra for all nine stars with results.

We estimated stellar masses and radii from spectroscopic parameters $\left(T_{\text {eff }}, \log g,[\mathrm{Fe} / \mathrm{H}]\right)$ by fitting them to a grid of models from the Dartmouth Stellar Evolution Database (Dotter et al. 2008). We used the isochrones Python package (Morton 2015a), which interpolates the Dartmouth model grid (mass-age- $[\mathrm{Fe} / \mathrm{H}]$ ) and estimates uncertainties via the emcee Markov Chain Monte Carlo (MCMC) package (ForemanMackey et al. 2013). This procedure gives mass and radius uncertainties as small as $\sim 2 \%$, not accounting for the intrinsic uncertainties of the Dartmouth models, which are most uncertain for cool stars. For $M_{\star}<0.8 M_{\odot}$, Feiden \& Chaboyer (2012) find that most Dartmouth evolution models agree with

\footnotetext{
${ }^{19}$ http://irtfweb.ifa.hawaii.edu/ spex/IRTF_Spectral_Library/
} 

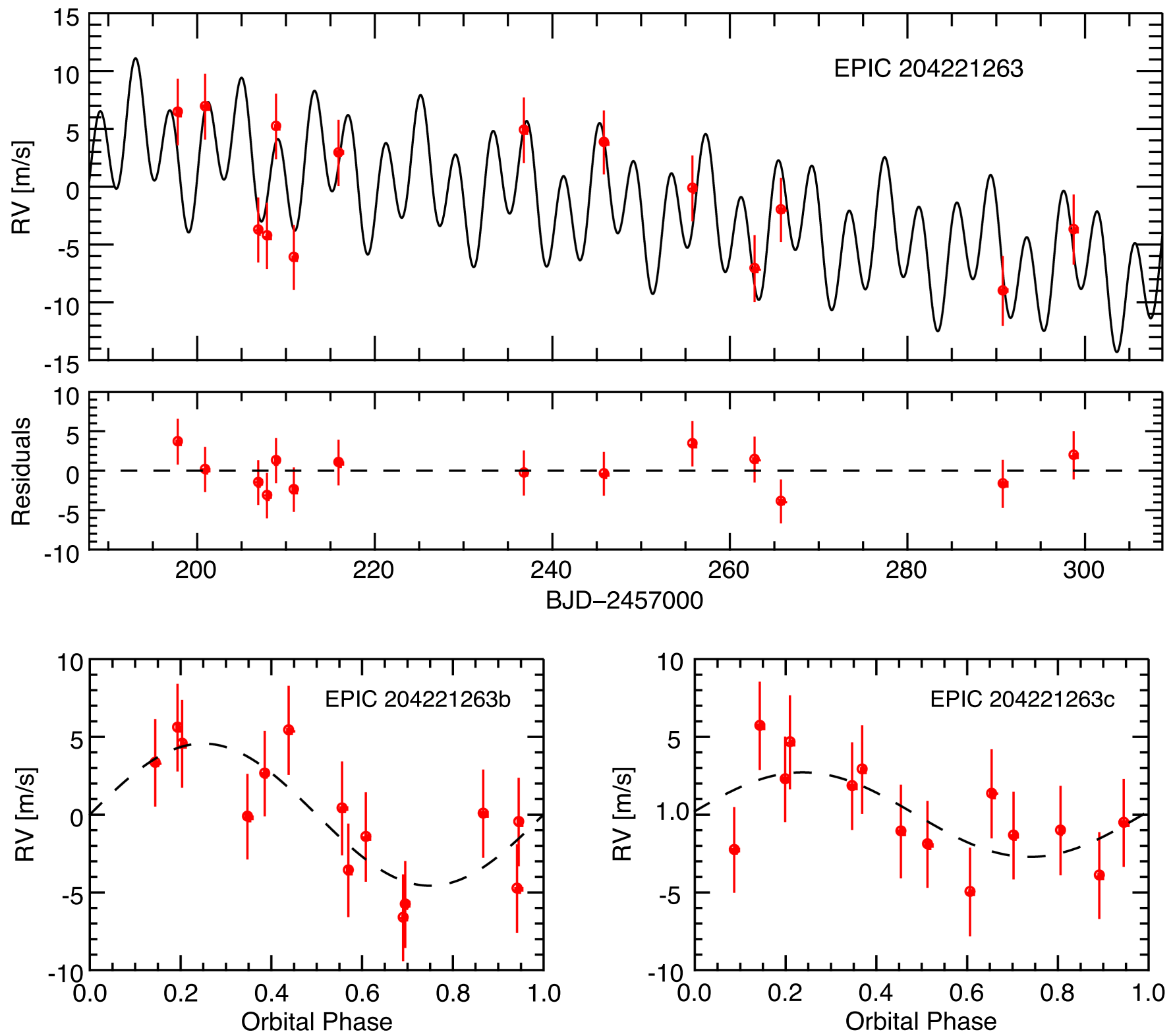

Figure 6. Top: RV time series observed with Keck/HIRES (red points), and the best two-planet fit, which includes a significant constant acceleration, $d v / d t$, evidence of a third bound companion at larger orbital distances. RV error bars represent the quadrature sum of individual measurement uncertainty and the best-fit jitter $\left(2.4 \mathrm{~m} \mathrm{~s}^{-1}\right)$. Bottom: RV time series of planets $\mathrm{b}$ (left) and $\mathrm{c}$ (right), folded at the orbital period of each planet with the linear trend and the Keplerian signal from the other planet subtracted. Transits occur at an orbital phase of 0.5 .

observed stellar radii to within $\sim 4 \%$. Therefore, for the nine stars analyzed with SpecMatch, we conservatively adopt minimum uncertainties for stellar mass and radius of 5\%. Our final stellar mass and radius uncertainties range from $\sim 5 \%$ to $10 \%$.

We also used the HIRES spectra to measure stellar activity. The HIRES spectra span the Ca II $\mathrm{H}$ and $\mathrm{K}$ lines (3969 A, $3934 \AA$ ) that are sensitive to chromospheric activity (Wilson 1968). Following Isaacson \& Fischer (2010), we measured $S_{\mathrm{HK}}$ indices - the ratio of flux in $\mathrm{Ca}$ II $\mathrm{H}$ and $\mathrm{K}$ line cores to flux in nearby continuum bands. These are converted into $\log R_{\mathrm{HK}}^{\prime}$ values (tabulated in Table 2), which account for differences in continuum flux levels with spectral type (Middelkoop 1982; Noyes et al. 1984). Since the conversion to $\log R_{\mathrm{HK}}^{\prime}$ is only calibrated for stars with $B-V<0.9\left(T_{\text {eff }} \sim 5000 \mathrm{~K}\right)$, we provide only $\log R_{\mathrm{HK}}^{\prime}$ values for stars $T_{\mathrm{eff}}>5000 \mathrm{~K}$ and $S_{\mathrm{HK}}$ values for the cooler stars. For reference, the Sun varies in the range $\log R_{\mathrm{HK}}^{\prime}=-4.85$ to -5.05 dex through the solar cycle (Meunier et al. 2010). K2-19 and K2-36 are moderately active $\left(\log R_{\mathrm{HK}}^{\prime}=-4.66 \mathrm{dex}\right.$ and $S_{\mathrm{HK}}=0.46$, respectively), while the other GK dwarfs are inactive.

Stellar parameters for the two cooler stars (K2-3 and K2-5) are derived from infrared spectra discussed in Section 2.4.2. Determination of stellar parameters for $\mathrm{K} 2-3$ is detailed in Crossfield et al. (2015). Here we discuss characterization of K2-5 using similar methods.

We used our SpeX spectrum to measure the near-IR $\mathrm{H}_{2} 0-\mathrm{K} 2$ index (Rojas-Ayala et al. 2012) to estimate a spectral type for $\mathrm{K} 2-5$ of $\mathrm{K} 7.5 \pm 0.5$. This index-based measurement is consistent with the visual best match presented in Figure 2. Following Crossfield et al. (2015) and Petigura et al. (2015), we estimated metallicity $([\mathrm{Fe} / \mathrm{H}])$, effective temperature $\left(T_{\text {eff }}\right)$, radius $\left(R_{\star}\right)$, and mass $\left(M_{\star}\right)$ using the methods presented in Mann et al. (2013a, 2013b). Metallicity is estimated using 


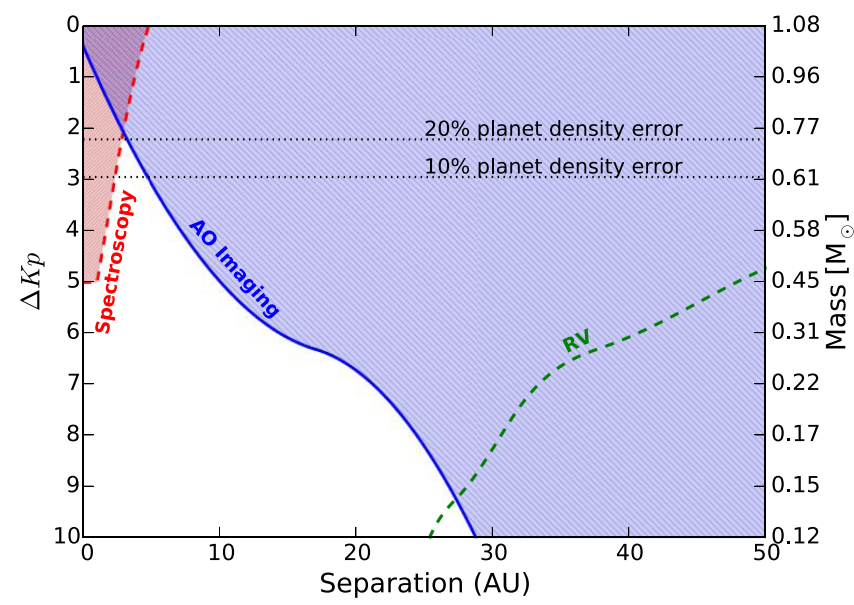

Figure 7. Constraints on the properties of an unseen companion. The axes denote a parameter space of brightness contrast of the companion with K2-38 (alternatively the mass of the companion) and the orbital separation between the two bodies. AO imaging excludes companions in the hatched blue region. The dashed red line shows the limits of our search for secondary lines in the high-resolution optical spectrum from HIRES. The dashed green line (lower right) corresponds to the masses and orbital semimajor axes consistent with the measured linear RV trend, assuming a circular, edge-on orbit and a companion mass much lower than that of the primary star (Equation (2)). The horizontal dashed lines represent companion contrasts at which the dilution of the observed transit depths would cause planet densities to be overestimated by $10 \%$ and $20 \%$. AO imaging and spectroscopy rule out companions that would cause systematic errors of $>20 \%$ in planet density with high confidence (see Section 6.2 for discussion).

spectroscopic index and equivalent width based methods (Rojas-Ayala et al. 2012; Terrien et al. 2012; Mann et al. 2013b) that were calibrated using a sample of $M$ dwarfs having wide, comoving FGK companions with well-determined $[\mathrm{Fe} / \mathrm{H}]$. We use IDL software made publicly available by A. Mann ${ }^{20}$ to calculate the $H$ - and $K$-band metallicities of K2-5. We average the $H$ and $K$ metallicities and add the measurement and systematic uncertainties in quadrature to arrive at the final value of $[\mathrm{Fe} / \mathrm{H}]=-0.33 \pm 0.20 \mathrm{dex}$. This star is metal-poor. Effective temperature, radius, and mass are calculated using temperature-sensitive spectroscopic indices in the JHK bands (Mann et al. 2013a) and empirical relations calibrated using nearby, bright $\mathrm{M}$ dwarfs with interferometrically measured radii (Boyajian et al. 2012). We calculated $T_{\text {eff }}$ in the $J H K$ bands and averaged the results. Conservative $T_{\text {eff }}$ uncertainties were estimated by adding in quadrature the rms scatter in the $J H K$-band values and the systematic errors in the empirical fits for each band (Mann et al. 2013a). The stellar radius and mass were computed using publicly available software from A. Mann. ${ }^{21}$ The resulting fundamental parameters for K2-5 are $T_{\text {eff }}=3930 \pm 375 \mathrm{~K}, R_{\star}=0.57 \pm 0.12$ $R_{\odot}$, and $M_{\star}=0.61 \pm 0.13 M_{\odot}$.

K2-5was presented as a multiplanet system in Montet et al. (2015b), where their fundamental parameters were estimated using broadband photometry and model fits. Our spectroscopic parameter estimates are consistent within uncertainties.

Table 2 lists spectroscopically derived stellar parameters.

\footnotetext{
${ }^{20}$ https://github.com/awmann/metal

${ }^{21}$ https://github.com/awmann/Teff_rad_mass_lum
}

\section{VALIDATION OF PLANET CANDIDATES}

There are several potential astrophysical events whose light curves can be confused with transiting planets. One example is a blended EB system, either bound to the primary or in the background of the target star's photometric aperture. Some of these astrophysical false positives can be distinguished from planet transits by secondary eclipses (SEs), but SEs do not always occur and are often undetectably small. Even if the primary star does host a planet, blending of other stars within the photometric aperture can dilute the transit depth, causing the planet radius to be underestimated (Ciardi et al. 2015). Follow-up observations are crucial for identifying any sources blended within the same 4 " Kepler pixels.

All of the objects presented in this catalog passed a series of complementary vetting tests: first, from the $K 2$ light curves, we identified EBs via their characteristic "V-shaped" dimming profiles and SEs. We also searched for nearby companions in AO images and archival images. In addition, we searched for multiple sets of stellar lines in the high-resolution optical spectra. We also estimate FPPs of each planet candidate, which are constrained by these follow-up observations.

Even without AO imaging or spectroscopy to screen for these blends, the FPPs are intrinsically lower compared to systems with a single planet candidate (Lissauer et al. 2012, 2014). For the prime Kepler mission the FPP for a single planet candidate system is $\sim 10 \%$ (Morton \& Johnson 2011; Fressin et al. 2013), but is reduced by factors of $\sim 25$ and $\sim 100$ for systems with one and two additional planet candidates, respectively (Lissauer et al. 2012). These FP rates apply to the prime Kepler mission and cannot be blindly applied to $K 2$, which has a different degree of source crowding, as well as different photometric noise properties, target selection criteria, and vetting procedures, all of which factor into the FP rate and "multiplicity boost" estimation.

In this section, we summarize the results of our AO and archival image searches, spectroscopic validation efforts, and FPP assessment. We estimate multiplicity boosts for $K 2$ fields $\mathrm{C} 1$ and $\mathrm{C} 2$ using available $K 2$ planet candidate catalogs.

\subsection{AO Imaging}

For each target star, our AO images were combined using a median average. Typical final FWHM resolutions were 4-6 pixels for a resolution of $\approx 0$ !" 05 with Keck/NIRC2 and $\approx 0$ ". 1 for Palomar/PHARO. For every target considered here, no other stars were detected within the fields of view of the cameras. For each final combined image, we estimated the sensitivities by injecting fake sources with an $\mathrm{S} / \mathrm{N}$ of 5 at distances of $N \times$ FWHM from the central source, where $N$ is an integer. The $5 \sigma$ sensitivities, as a function of radius from the stars, are shown in Figure 3 along with a full field-of-view combined image. Typical sensitivities yield contrasts of 2-3 mag within 1 FWHM of the target star and contrasts 4-6 mag within 3-4 FWHM. In the "flat" ( $>6$ FWHM) of the image, the typical contrasts were 8-9 mag fainter than the target star.

\subsection{Archival Imaging}

We also searched for neighboring stars at separations beyond the edges of our AO images (typically $10^{\prime \prime}$ ), but within the $K 2$ photometric apertures (typically $10^{\prime \prime}-15^{\prime \prime}$ ). We downloaded $60^{\prime \prime} \times 60^{\prime \prime} r_{\mathrm{P} 1}$-band images from the Pan-STARRS1 $3 \pi$ survey (Kaiser et al. 2010), surrounding each of the EPIC target stars. 

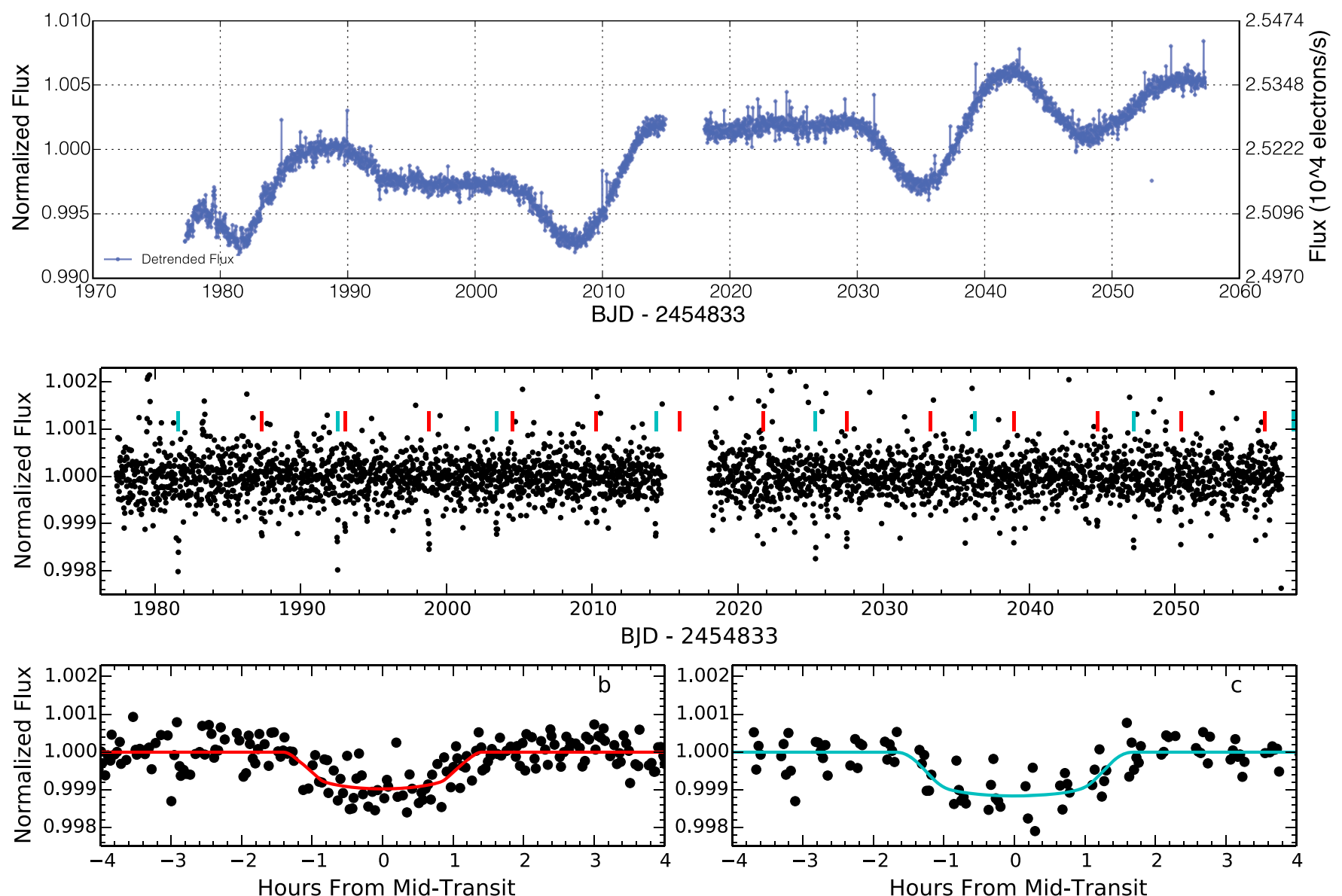

Figure 8. Top: $K 2$ photometry for K2-5 after subtracting variations caused by telescope roll. Middle: calibrated $K 2$ photometry. Vertical ticks indicate times of planet transits. Bottom: phase-folded photometry and best-fitting light curves for each planet.

The images have a plate scale of 0.25 pixel $^{-1}$ and average seeing-limited resolution of $\sim 1^{\prime \prime}$. The target stars are generally saturated in these images, but background sources can be easily identified down to a limiting magnitude of $r_{\mathrm{P} 1} \approx 23 \mathrm{mag}$. We used the magnitude zero points in the FITS headers and performed our own aperture photometry on the images to estimate the magnitudes of nearby sources. Secondary sources are detected within the $K 2$ aperture of four systems. For three of these systems (K2-19, K2-37, K2-32) secondary sources were bright enough to produce observed transit depths. In these three cases, we regenerated the light curves using smaller apertures that excluded those other stars and verified that the transit signals remained. We note that all listed transit parameters derive from light curves produced with the original (larger) apertures because of reduced photometric noise. All secondary sources are sufficiently faint such that dilution corrections would have negligible effects on measured transit depths-correction factors would be more than an order of magnitude less than uncertainties on $R_{p} / R_{\star}$. An analysis of each EPIC target is given below.

$K 2-5$ : No sources fall within the $12^{\prime \prime}$ aperture to a limiting magnitude of $r_{\mathrm{P} 1} \approx 23 \mathrm{mag}$. The nearest bright source is $15^{\prime \prime}$ to the NE with $r_{\mathrm{P} 1}=18.9 \mathrm{mag}$.

K2-3: No stellar sources fall within the $16^{\prime \prime}$ aperture to a limiting magnitude of $r_{\mathrm{P} 1} \approx 22 \mathrm{mag}$. The nearest star detected in the Pan-STARRS1 imaging is $26^{\prime \prime}$ to the NE with $r_{\mathrm{P} 1}=17.2 \mathrm{mag}$.
K2-8: No sources fall within the $12^{\prime \prime}$ aperture to a limiting magnitude of $r_{\mathrm{P} 1} \approx 22$ mag. No stars or galaxies brighter than $r_{\mathrm{P} 1}=21.6 \mathrm{mag}$ fall within $30^{\prime \prime}$ of the target.

K2-19: One faint star falls within the $12^{\prime \prime}$ aperture 10 !' 7 to the NWW with $r_{\mathrm{P} 1}=20.7 \mathrm{mag}$. We estimate that this source is contributing $0.6 \mathrm{ppt}$ to the $K 2$ photometry. An eclipse of the secondary source would not be deep enough to produce the observed transits of K2-19b or K2-19c. Moreover, Narita et al. (2015) measure a contrast of $\sim 0.1 \mathrm{ppt}$ in $H$ band and detect the transits of K2-19b and K2-19c when the faint star lies outside the photometric aperture, localizing them to the primary. However, an eclipse of the secondary could produce the observed $0.1 \mathrm{ppt}$ transits of K2-19d. We re-extracted the photometry using an $8^{\prime \prime}$ aperture, small enough to exclude the faint nearby source. The transit signals of all three planets were detected, and their depths were consistent with those measured using the original (larger) aperture. The transits of all three planets are therefore localized to the bright star of interest. Dilution correction factors are negligible compared to measurement uncertainties on $R_{p} / R_{\star}$, so we do not apply them.

K2-35: No sources fall within the $12^{\prime \prime}$ aperture to a limiting magnitude of $r_{\mathrm{P} 1} \approx 22 \mathrm{mag}$. There are two nearby stars just outside the $K 2$ aperture. One star is $22^{\prime \prime}$ to the WNW with $r_{\mathrm{P} 1}=15.1 \mathrm{mag}$, and the other is $27^{\prime \prime}$ to the NNW with $r_{\mathrm{P} 1}=14.3 \mathrm{mag}$. 

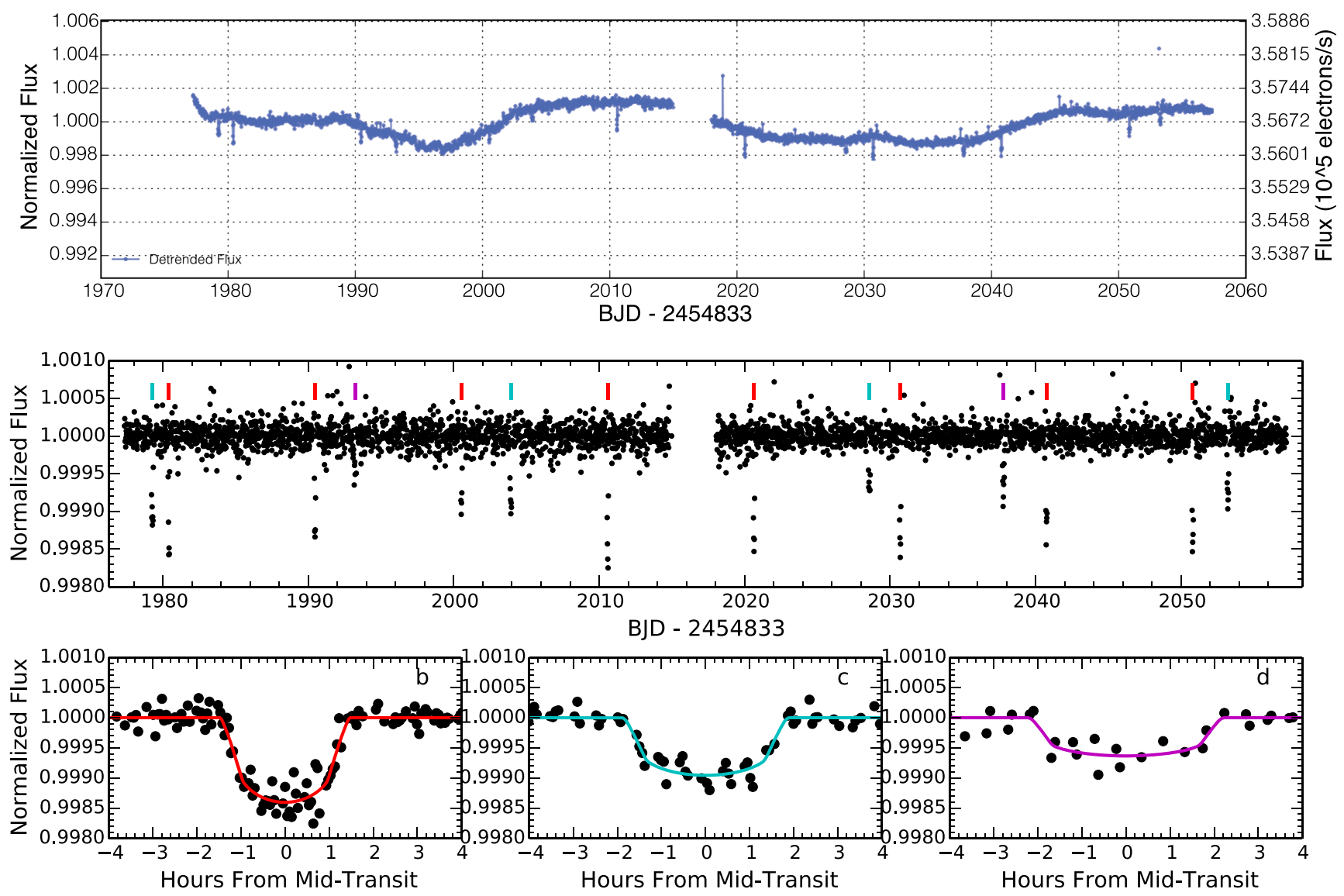

Figure 9. Top: K2 photometry for K2-3 after subtracting variations caused by telescope roll. Middle: calibrated K2 photometry. Vertical ticks indicate times of planet transits. Bottom: phase-folded photometry and best-fitting light curves for each planet.

K2-36: There are two very bright sources $24^{\prime \prime}$ to the NE and $24^{\prime \prime}$ to the SE of K2-36, but both fall outside the $12^{\prime \prime} K 2$ aperture. These sources are saturated in the Pan-STARRS1 data but are of comparable brightness to K2-36.

K2-16: No sources fall within the $8^{\prime \prime} K 2$ aperture to a limiting magnitude of $r_{\mathrm{P} 1} \approx 22 \mathrm{mag}$. The nearest detected source is $29^{\prime \prime}$ to the $\mathrm{W}$ with $r_{\mathrm{P} 1}=20.3 \mathrm{mag}$.

$K 2-24$ : One faint star falls within the $12^{\prime \prime} K 2$ aperture $6^{\prime \prime}$ to the $\mathrm{W}$ of the target. This star is badly blended with the wings of the saturated point-spread function (PSF) of K2-24, so reliable photometry cannot be extracted. However, we estimate that the star is no brighter than $r_{\mathrm{P} 1} \approx 18.5 \mathrm{mag}$, which would contribute only $0.8 \mathrm{ppt}$ to the light in the $K 2$ aperture and could not be the source of the $2 \mathrm{ppt}$ and $4 \mathrm{ppt}$ transits of K2-24b and K2-24c, respectively. We do not apply a dilution correction to the measured transit depths because it would have a negligible effect.

K2-37: Two other stars fall within the $12^{\prime \prime}$ aperture. One star, $r_{\mathrm{P} 1}=19.1 \mathrm{mag}$, is located 9!" 4 to the ESE, and the other, $r_{\mathrm{P} 1}=19.8 \mathrm{mag}$, is 8.13 to the WNW. Combined, the contaminating sources contribute $3 \mathrm{ppt}$ to the flux in the $K 2$ aperture. We regenerated the photometry using an $8^{\prime \prime}$ aperture that excluded the two other stars, and the transits were still visible, confirming that the primary star is being transited. We do not correct the transit depths for dilution as this would have a negligible effect.

$K 2$-38: No stars brighter than $r_{\mathrm{P} 1} \approx 20.3$ mag fall within the $12^{\prime \prime} K 2$ aperture. The nearest comparably bright source is an $r_{\mathrm{P} 1}=19.1 \mathrm{mag}$ star $29^{\prime \prime}$ to the NW.
$K 2-32$ : Several faint sources fall within the $16^{\prime \prime} K 2$ aperture. The brightest of these is $15^{\prime \prime}$ to the south with $r_{\mathrm{P} 1}=18.9 \mathrm{mag}$. This contaminating source contributes $2 \mathrm{ppt}$ to the $K 2$ aperture flux and is bright enough to account for the transit depths of planets $\mathrm{c}$ and $\mathrm{d}$ but not planet $\mathrm{b}$. We re-extracted the photometry using an $8^{\prime \prime}$ aperture, small enough to exclude the other nearby stars, and the transits were still visible. The transits are therefore localized to the target of interest. Changes in transit depths caused by dilution from the secondary sources are negligible.

\subsection{Spectroscopic Vetting}

We searched the HIRES spectra for multiple sets of stellar lines using the algorithm of Kolbl et al. (2015). The algorithm is sensitive to blends from secondary stars in the $0 . " 87 \times 14^{\prime \prime}$ HIRES slit that have effective temperatures $T_{\text {eff }}=3400-6100$ $\mathrm{K}$, have brightness ratios $\gtrsim 1 \%$ in $V$ and $R$ bands, and differ in $\mathrm{RV}$ by $\gtrsim 10 \mathrm{~km} \mathrm{~s}^{-1}$ from the primary star. For the 10 targets with HIRES spectra, no spectroscopic blends were detected.

\subsection{False-positive Assessment}

We estimate the FPP of each planet candidate signal using the Python package VESPA (Morton 2015b). We supply VESPA with the phase-folded $K 2$ light curve; photometry from APASS, the Two Micron All Sky Survey (2MASS), and WISE; stellar parameters derived from spectroscopy (Section 3); the contrast curve from AO imaging (Section 4.1); and the maximum allowed contrast and velocity offset determined by 

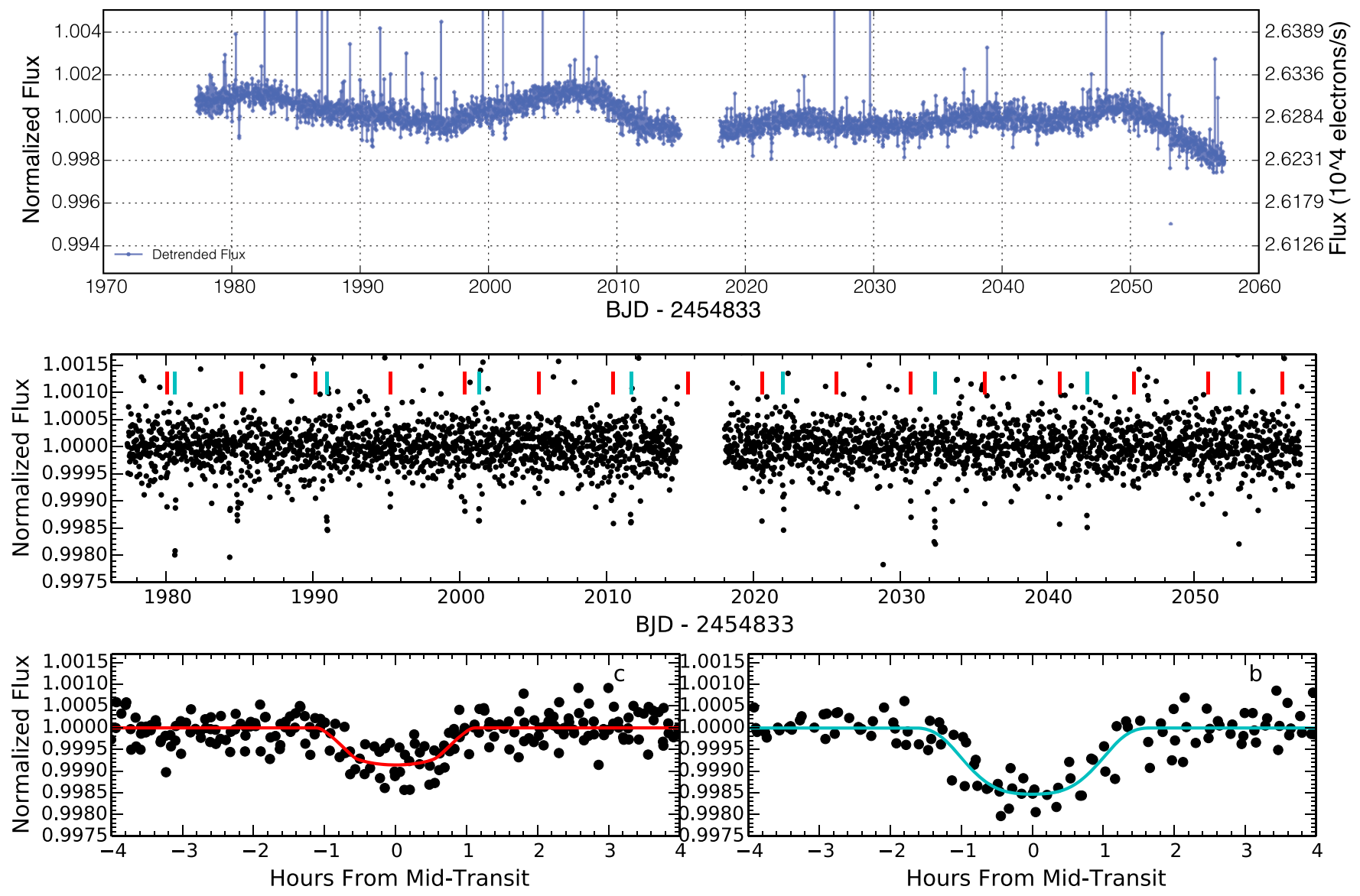

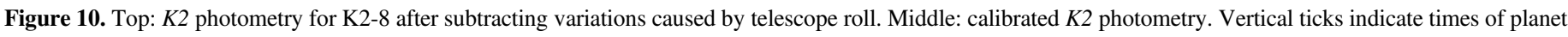
transits. Bottom: phase-folded photometry and best-fitting light curves for each planet.

our spectroscopic vetting (Section 4.3). Given these constraints, VESPA estimates the FPP - the likelihood that the transit signal was produced by a true planet around the target star and not by an EB, hierarchical triple system, or nonassociated star with a transiting planet. The FPPs returned by VESPA are listed in Table 3 along with planet candidate dispositions. Dispositions take into account this study and other previously published studies.

For planet candidates that are not "confirmed" by mass detection, we rely on computing an FPP as a means of validation. Following Montet et al. (2015b), we assign a "validated" disposition to planet candidates with FPP $<1 \%$. All planet candidates are validated by VESPA to better than 99\% confidence, except for K2-8b and K2-32c, which have FPPs of $1.3 \%$ and $2.2 \%$, respectively. However, these estimates neglect the "multiplicity boost"-an additional factor of confidence in the planet hypothesis gained from the detection of multiple planet candidates in these systems. For the prime Kepler mission, Lissauer et al. (2012) estimate that the a priori FPP is $\sim 50-100 \times$ lower for systems with three or more planet candidates and $\sim 25 \times$ lower for systems with two planet candidates compared to those with one planet candidate. These were derived using two different methods, each of which assumes that false positives (FPs) are randomly distributed among Kepler targets, and that the presence of FPs and detectable planet signals are uncorrelated.
We apply the same methods to $K 2$ planets. Following Lissauer et al. (2012), for a system with two planet candidates, if $P_{1}$ is the probability of a candidate's planethood before considering multiplicity, then the probability of planethood after accounting for multiplicity is

$$
P_{2} \approx \frac{X_{2} P_{1}}{X_{2} P_{1}+\left(1-P_{1}\right)},
$$

where $X_{2}$ is the "multiplicity boost" for systems of two planet candidates. K2-32c is part of a three-candidate system, but, for argument sake, it is sufficient to assume that the multiplicity boost for three-candidate systems will be at least as large as that for two-candidate systems. Lissauer et al. (2012) estimate $X_{2}$ using two different methods.

The first method compares the fraction of Kepler targets with planet candidates $\left(F_{\text {cand }} \sim 1 / 150\right)$ to the fraction of planet candidate hosts with more than one planet candidate $\left(F_{\text {multi }} \sim\right.$ $1 / 6)$. If planets and FPs were randomly distributed among Kepler targets, the detection rate of Kepler multis would be much lower $\left(F_{\text {multi }} \sim F_{\text {cand }}\right)$. Assuming that FPs are randomly distributed and that planets are not, Lissauer et al. (2012) estimate $X_{2} \sim F_{\text {multi }} / F_{\text {cand }}=25$. We make the same assumptions to estimate $X_{2}$ for $K 2$ fields $\mathrm{C} 1$ and $\mathrm{C} 2$ using the catalog of Vanderburg et al. (2016), who adopt a transit detection threshold of $\mathrm{S} / \mathrm{N}>9$. We assume that the target 

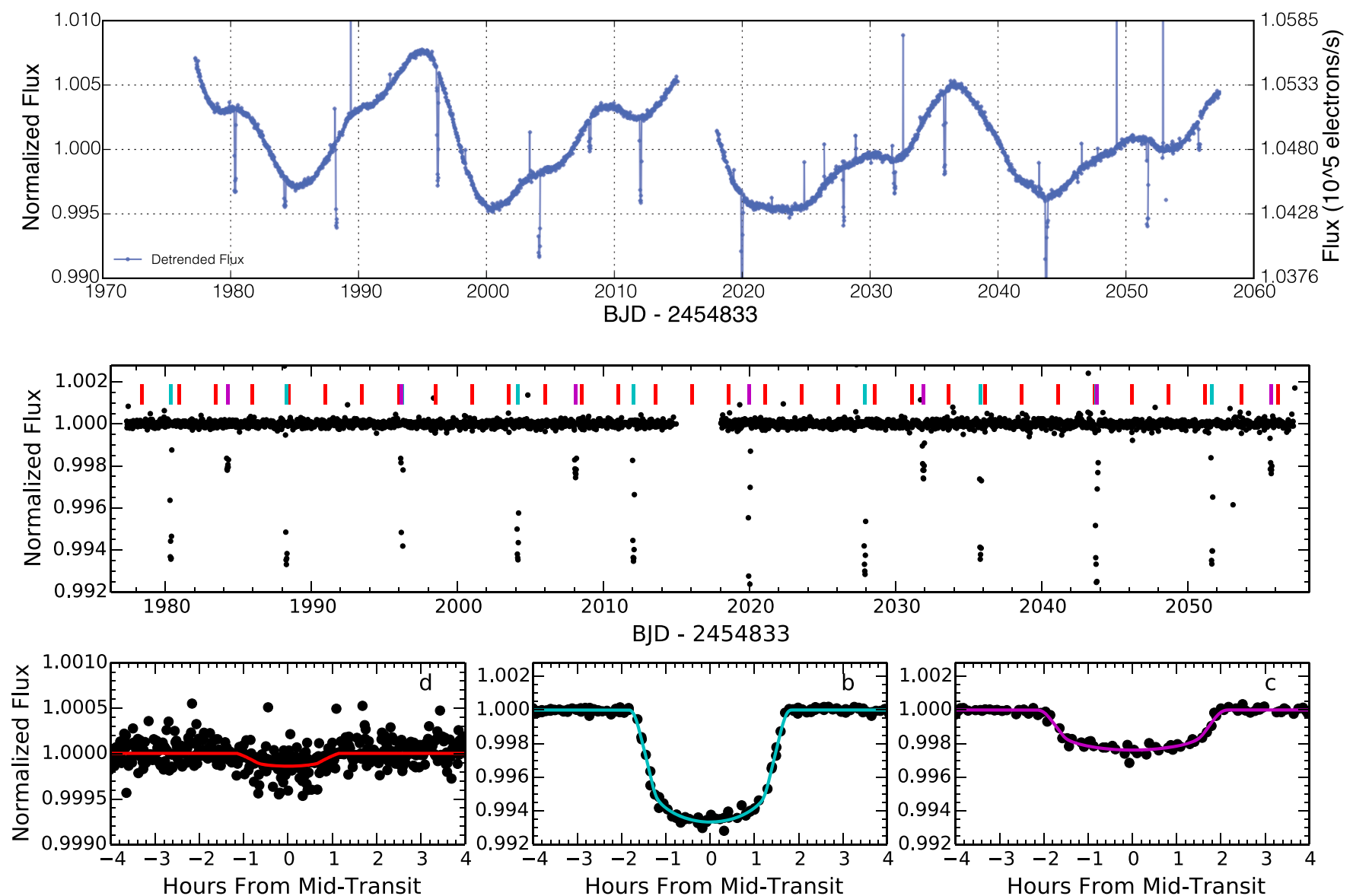

Figure 11. Top: $K 2$ photometry for K2-19 after subtracting variations caused by telescope roll. Middle: calibrated $K 2$ photometry. Vertical ticks indicate times of planet transits. Bottom: phase-folded photometry and best-fitting light curves for each planet.

sample in a given $K 2$ field consists of all objects denoted as "STAR" in the EPIC catalog. Combining $\mathrm{C} 1$ and $\mathrm{C} 2$, we compute $F_{\text {cand }} \sim(116 / 32,264)=0.4 \%$ and $F_{\text {multi }} \sim(10 /$ $116)=8.6 \%$. These suggest $X_{2} \sim 24$, similar to Kepler. Substituting $X_{2}=24$ into Equation (1) and setting $P_{1}$ according to our VESPA constraints give corrected FPPs (1 $\left.P_{2}\right)=0.06 \%$ and $0.09 \%$ for $\mathrm{K} 2-8 \mathrm{~b}$ and $\mathrm{K} 2-32 \mathrm{c}$, respectively. We repeated these estimates for $\mathrm{C} 1$ and $\mathrm{C} 2$ independently, with similar results. We also applied these methods to our own catalog of $\sim 100$ planet candidates in $\mathrm{C} 1$ and $\mathrm{C} 2$ detected by TERRA (Crossfield et al. 2016), requiring $\mathrm{S} / \mathrm{N}>12$ and three transits. This yields $X_{2} \sim 34$ and corrected FPPs $(1-$ $\left.P_{2}\right)=0.04 \%$ and $0.07 \%$ for $\mathrm{K} 2-8 \mathrm{~b}$ and $\mathrm{K} 2-32 \mathrm{c}$, respectively. The multiplicity boosts estimated using either catalog are an order of magnitude larger than those needed to validate $\mathrm{K} 2-8 \mathrm{~b}$ and K2-32c to better than $99 \%$ confidence.

As an additional check, we estimate the multiplicity boost using a second method of Lissauer et al. (2012). This method assumes that some fraction of candidates $F_{\text {true }}$ are true planets in order to estimate the expected fraction of multicandidate systems that have at least one FP. Note that $F_{\text {true }}$ is denoted as $P$ in Lissauer et al. (2012), Equations (2) and (4). Those two equations are used to estimate the expected number of FPs in two-candidate systems based on $F_{\text {true }}$ and the total numbers of observed targets and planet candidates. Subsequently dividing by the number of candidates in two-planet systems yields the fraction of candidates in two-candidate systems expected to be true planets. Using this method, Lissauer et al. (2012) estimated $X_{2} \sim 25$ for the prime Kepler mission, consistent with the first method above. We apply this same method to our own catalog of $K 2$ planet candidates - unlike Vanderburg et al. (2016), we compute FPPs for all candidates, most of which have been vetted via spectroscopy and high-resolution imaging. By integrating over all FPPs, we estimate $F_{\text {true }} \sim 90 \%$ and $\sim 60 \%$ and $X_{2} \sim 70$ and $\sim 20$ for $\mathrm{C} 1$ and $\mathrm{C} 2$, respectively. These are similar to the prime Kepler mission $\left(F_{\text {true }} \sim 90 \%\right)$. Plugging these $X_{2}$ values into Equation (1) and setting $P_{1}$ according to our VESPA constraints yields corrected FPPs (1 $\left.P_{2}\right)=0.02 \%$ and $0.12 \%$ for $\mathrm{K} 2-8 \mathrm{~b}$ and $\mathrm{K} 2-32 \mathrm{c}$, respectively. For this method, the multiplicity boost is still effective at validating these two planet candidates to FPP $<1 \%$ as long as $F_{\text {true }} \gtrsim 15 \%$.

In summary, the multiplicity boosts estimated via both methods, when combined with VESPA constraints, are large enough to validate $\mathrm{K} 2-8 \mathrm{~b}$ and $\mathrm{K} 2-32 \mathrm{c}$ to much better than $99 \%$.

\section{DERIVED PLANET PROPERTIES}

Our light-curve analysis follows Crossfield et al. (2015), which we summarize here. Starting with the detrended light curves from TERRA (Section 2.2), we perform a sliding median 

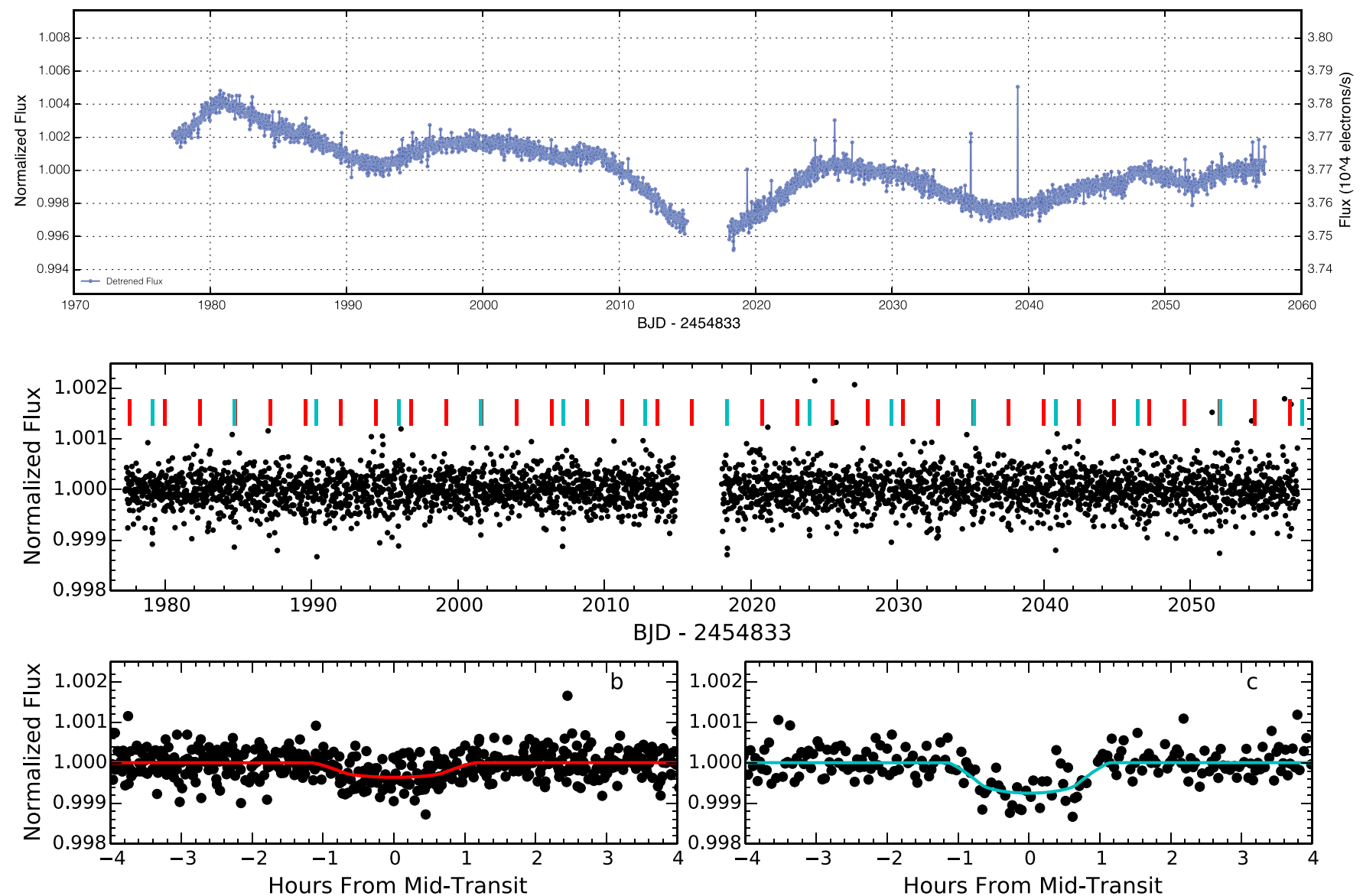

Figure 12. Top: $K 2$ photometry for K2-35 after subtracting variations caused by telescope roll. Middle: calibrated $K 2$ photometry. Vertical ticks indicate times of planet transits. Bottom: phase-folded photometry and best-fitting light curves for each planet.

subtraction to remove variability on several-day timescales, including stellar modulation. We fit JKTEBOP transit models (Southworth et al. 2004; Southworth 2011) to the light curves, using the emcee MCMC package (Foreman-Mackey et al. 2013) to generate posterior probability distributions for the transit model parameter. We use the best-fitting transit depth, phase, and orbital period from TERRA for the initial model guess.

We assume circular orbits and adopt a linear limb-darkening model, imposing a Gaussian prior on the limb-darkening coefficient $u$. The mean of this Gaussian is selected by interpolating the limb-darkening tables of Claret et al. (2012, 2013) to our spectroscopically measured $T_{\text {eff }}$ and $\log g$. The standard deviation was taken to be 0.05 . We tested standard deviations of 0.1 and also tried propagating our $T_{\text {eff }}$ and $\log g$ uncertainties through the interpolation procedure, but our results were insensitive to the chosen method. The use of a quadratic limb-darkening model also resulted in negligible changes to the posteriors.

Detrended light curves, fitted transit models, and derived planet parameters are presented for all 11 systems in Figures 818 and Tables 6-16. Derived parameters include orbital distance $a$, incident flux $S_{\oplus}$, and equilibrium temperature $T_{\text {eq }}$. The discoveries of K2-3 and K2-24 planets are reported in Crossfield et al. (2015) and Petigura et al. (2016), based on the same data products and analysis methods presented here. We include them in our catalog for completeness. All stellar and planet parameters will be provided in an online supplementary table.

Table 3 lists key parameters for each multiplanet system. The 26 planets are plotted in radius versus orbital period in Figure 4 . The points are colored according to host stellar mass. Twenty-one of the planets are likely smaller than Neptune $\left(R_{p}<3.8 R_{\oplus}\right)$.

Figure 5 displays the architectures of all systems. Systems are ordered top to bottom by decreasing orbital period of the inner planet. The largest planet in each system is colored red, the second-largest planet is green, and the third-largest planet (if present) is blue. This ranking scheme considers posterior medians and does not account for uncertainties, thus providing the most likely ranking. In six out of seven systems having only planets $R_{p}<3 R_{\oplus}$, planet size increases with $P$.

\section{MASSES OF K2-38 SUPER-EARTHS}

\subsection{Doppler Measurements}

In an initial campaign with Keck/HIRES, we obtained 14 RV measurements of K2-38 between 2015 June 24 UT and 2015 October 3 UT. These observations followed the standard procedures of the CPS (Howard et al. 2010). We used the "C2" decker $\left(0\right.$ !" $87 \times 14^{\prime \prime}$ slit $)$ with a cell of molecular iodine gas placed in front of the spectrometer slit to imprint a dense set of molecular absorption lines on the stellar spectrum, subjected to the same instrumental effects. Exposure times were typically 

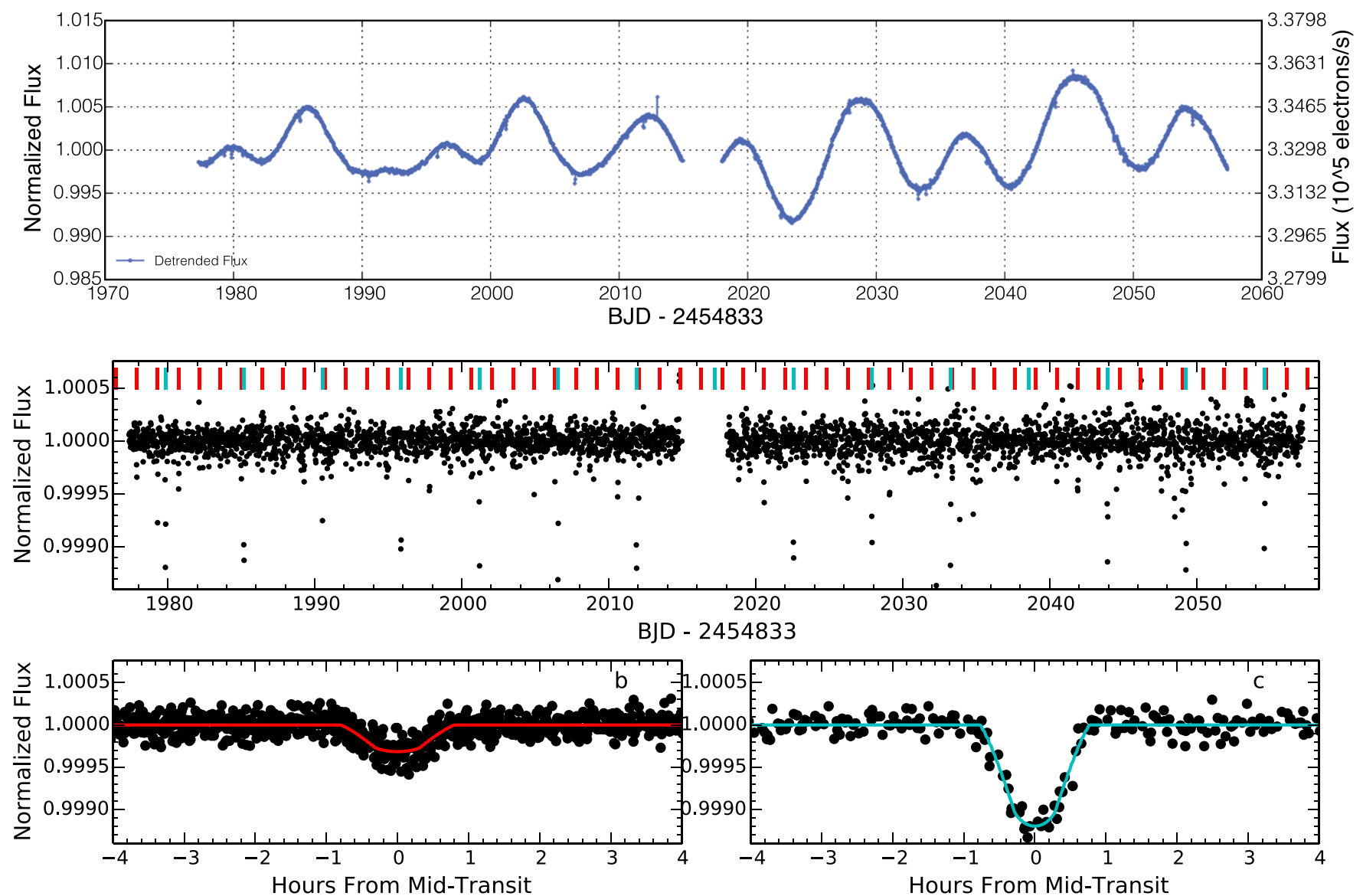

Figure 13. Top: $K 2$ photometry for K2-36 after subtracting variations caused by telescope roll. Middle: calibrated $K 2$ photometry. Vertical ticks indicate times of planet transits. Bottom: phase-folded photometry and best-fitting light curves for each planet.

$\sim 20$ minutes and were determined by an exposure meter that terminated exposures when an $\mathrm{S} / \mathrm{N}$ pixel $^{-1}$ of 160 in the continuum near $550 \mathrm{~nm}$ was reached. The iodine lines serve as a wavelength reference and calibration for the PSF over the entire spectral formal. We also gathered an iodine-free spectrum with the "B3" decker $\left(0\right.$ " $57 \times 14^{\prime \prime}$ slit $)$. RVs were determined by forward-modeling the iodine-free spectrum, a high-resolution/high-S/N spectrum of the iodine transmission, and the instrumental response (Marcy \& Butler 1992; Valenti et al. 1995; Butler et al. 1996; Howard et al. 2009). Our measured RVs are listed in Table 4. Individual RV measurement uncertainties are in the range $1.3-1.8 \mathrm{~m} \mathrm{~s}^{-1}$.

Figure 6 shows the measured RV time series for K2-38. The star has low astrophysical jitter, and we were able to make initial mass measurements of the two planets. We fit a twoplanet model using the IDL package RVLIN (Wright \& Howard 2009). Our RV model assumes two planets with circular orbits, with the orbital periods and phases fixed to the values measured from transits. We used a likelihood function constructed as in Howard et al. (2014). The model has five free parameters, including the RV semi-amplitude of planets b and c, $K_{b}$ and $K_{c}$, a constant RV offset, $\gamma$, and a constant radial acceleration (RV changing linearly with time), $d v / d t$. We also include an RV "jitter" parameter, $\sigma_{\text {jitter, }}$, to account for additional Doppler noise, which might have astrophysical or instrumental origins. We estimate a jitter of $2.4_{-0.7}^{+1.0} \mathrm{~m} \mathrm{~s}^{-1}$, which is consistent with expectations for old, solar-type stars (Wright 2005; Isaacson \& Fischer 2010).

The RV time series has a negative slope, suggesting that we are seeing a small orbital segment of a third companion with a wider orbital separation. To test this hypothesis, we compared models with and without the constant RV acceleration parameter using the Bayesian Information Criterion (BIC; Schwarz 1978; Liddle 2007). Comparing the the best-fitting models, we find $\mathrm{BIC}_{d v / d t}-\mathrm{BIC}_{d v / d t=0}=-5.6$, indicating that the model that includes constant acceleration is strongly preferred. There is very likely to be a third, more distant companion in this system, and we constrain its properties in Section 6.2. Additional RV measurements in early 2016, when the target is next observable, will provide a stronger test of the long-term trend.

Using the same RV model and likelihood function, we performed an MCMC analysis of the RVs to determine parameter uncertainties. We used emcee (Foreman-Mackey et al. 2013) and adopted Gaussian priors on the orbital periods and phases, with means and widths matching the posteriors derived from fitting the light curve (Section 5). We adopted uninformed priors for $K_{b}, K_{c}, \sigma_{\text {jitter }}, \gamma$, and $d v / d t$. By allowing the model to explore unphysical solutions with $K<0$, we did not bias the analysis to positive planet masses. We used the best fits from RVLIN to initialize the process. We discarded the first 500 steps. Every 2000 MCMC steps thereafter, we computed the Gelman-Rubin statistic (GRS; Gelman \& Rubin 1992) to assess convergence. We adopted convergence 

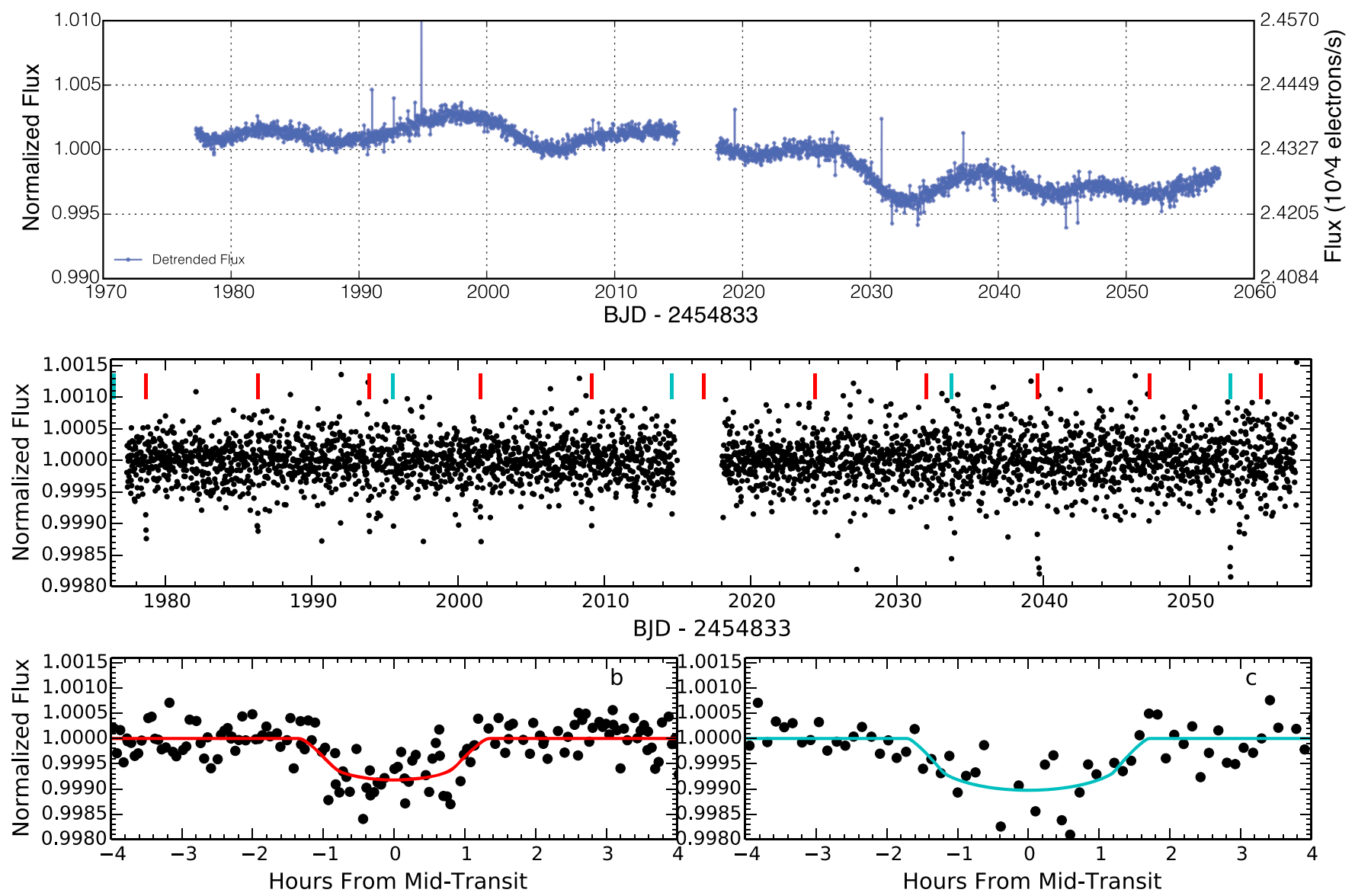

Figure 14. Top: $K 2$ photometry for K2-16 after subtracting variations caused by telescope roll. Middle: calibrated $K 2$ photometry. Vertical ticks indicate times of planet transits. Bottom: phase-folded photometry and best-fitting light curves for each planet.

criterion GRS $<1.03$ and generated posteriors once this condition was satisfied.

Table 5 lists measured RV semi-amplitudes, masses, and bulk densities of $\mathrm{K} 2-38 \mathrm{~b}$ and $\mathrm{K} 2-38 \mathrm{c}$. We list posterior medians, with quoted uncertainties being 15.87 and 84.13 percentiles. For the inner planet, we measure a mass of $12.0 \pm 2.9 M_{\oplus}$. Combining this with our planet radius measurement gives a bulk density of $17.5_{-6.2}^{+8.5} \mathrm{~g} \mathrm{~cm}^{-3}$. The mass and bulk density of the outer planet are $9.9 \pm 4.6 M_{\oplus}$ and $3.6_{-1.9}^{+2.7}$ $\mathrm{g} \mathrm{cm}^{-3}$, respectively. We discuss possible compositions for both planets in Section 8.

The marginalized posterior distribution for the linear trend gives $d v / d t=-37 \pm 11 \mathrm{~m} \mathrm{~s}^{-1} \mathrm{yr}^{-1}$. This linear trend contributes a change in RV of $\sim 10 \mathrm{~m} \mathrm{~s}^{-1}$ over the $\sim 100$-day time baseline of our RV campaign. This suggests a Keplerian signal with semi-amplitude $K \gtrsim 5 \mathrm{~m} \mathrm{~s}^{-1}$ and $P \gtrsim 200$ days ( $a$ $\gtrsim 0.7 \mathrm{AU})$.

\subsection{Constraints on an Additional Body}

We considered the possibility that the source of the linear $\mathrm{RV}$ trend is a companion star that contributes enough light to the $K 2$ photometry to significantly dilute the observed transit depths. In this scenario we would underestimate the planet radii and overestimate the planet densities (Ciardi et al. 2015). We assessed this possibility using our AO images and HIRES spectra. We confined the companion properties to a small domain of companion mass (or contrast with the primary) and orbital separation. These constraints are summarized in Figure 7 and suggest a low likelihood that the companion is bright enough to affect our measured density by more than $20 \%$. The nondetection of secondary lines in the HIRES spectrum allows us to exclude stars close in proximity and mass to the primary, specifically $\Delta K p \leqslant 5$ and $\Delta \mathrm{RV} \gtrsim 10 \mathrm{~km} \mathrm{~s}^{-1}$ (red dashed line). The plotted boundary (dashed red line) assumes that the HIRES spectrum was acquired at an orbital phase of maximum $\Delta \mathrm{RV}$. Our Keck/NIRC2 AO contrast curve (blue solid line) extends the exclusion region to fainter companions at larger separations. Horizontal dotted lines show stellar companions that would cause planet densities to be overestimated by $10 \%$ and $20 \%$. There is a small window of unvetted parameter space, spanning companion masses of $\sim 0.6-0.7 M_{\odot}$ and orbital separations $\sim 4-5 \mathrm{AU}$, which would cause planet densities to be overestimated by $10 \%-20 \%$ (planet radii underestimated by $3 \%$ and $6 \%$, respectively). This potential underestimate is smaller than our measurement uncertainties. There are a few noteworthy caveats: if the AO imaging happened to take place when the projected separation was small, then a brighter companion could go undetected. The same applies if the HIRES spectrum was taken when the difference between the RVs of the primary star and its companion was low or if the orbit is near face-on (misaligned with the transiting planets). 

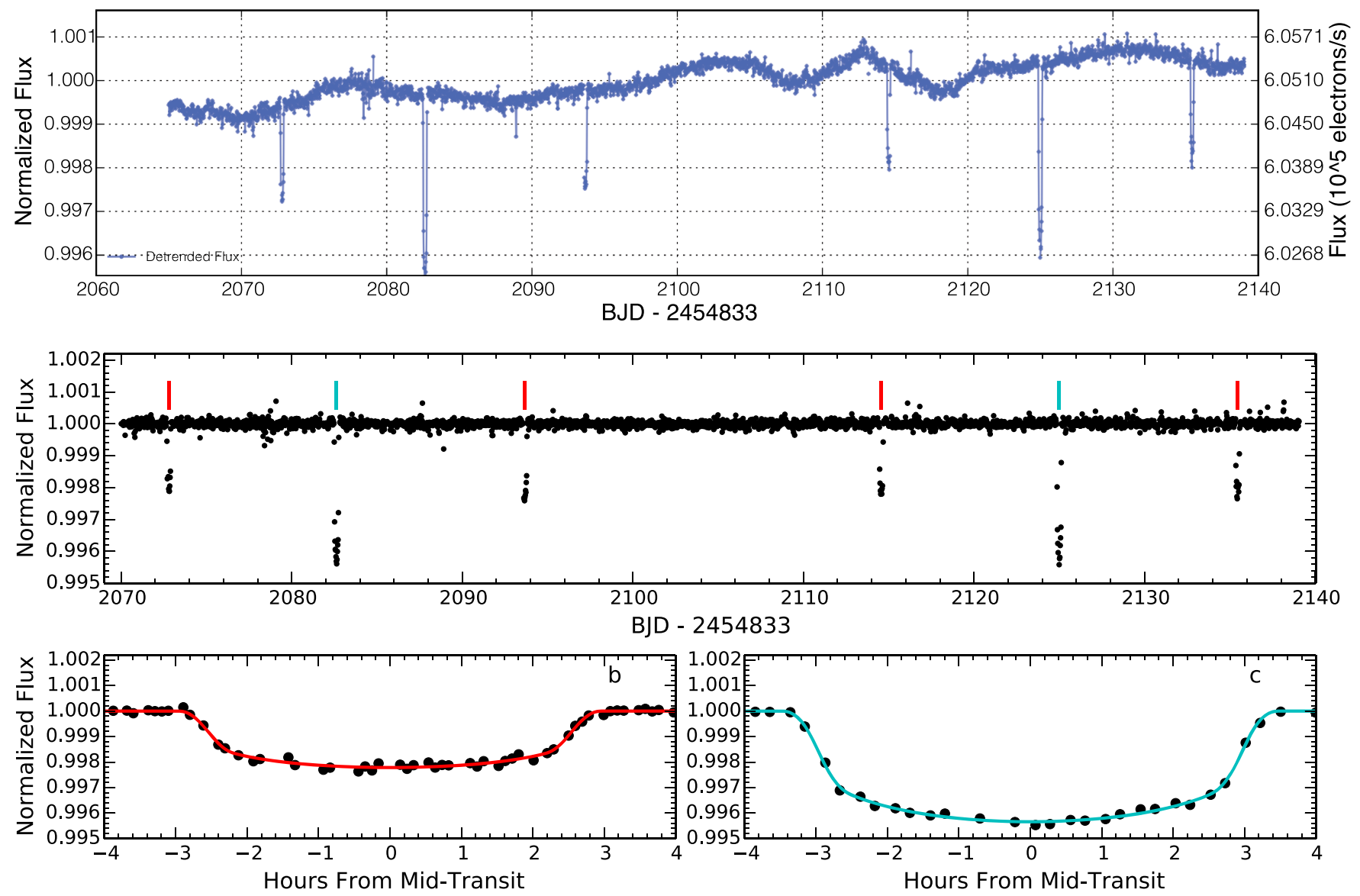

Figure 15. Top: $K 2$ photometry for K2-24 after subtracting variations caused by telescope roll. Middle: calibrated $K 2$ photometry. Vertical ticks indicate times of planet transits. Bottom: phase-folded photometry and best-fitting light curves for each planet.

Note that while a near face-on orbit would limit spectroscopic constraints, it would maximize detectability by AO imaging.

For the above analysis, we used riJHK photometric calibrations of Kraus \& Hillenbrand (2007) to convert angular separation to orbital distance $(\sim 170 \mathrm{pc})$ and to convert contrasts in the NIRC2 AO bandpass to contrasts in the Kepler bandpass and to companion masses. Following Winn et al. (2010), if we assume that the companion has a circular orbit and mass $M_{2} \ll$ $M_{\star}$, then our measured $d v / d t=-37 \pm 11 \mathrm{~m} \mathrm{~s}^{-1} \mathrm{yr}^{-1}$ implies

$$
M_{2} \sin i \sim 0.2 M_{\mathrm{Jup}}\left(\frac{a}{1 \mathrm{AU}}\right)^{2}
$$

where $M_{\mathrm{Jup}}$ is the mass of Jupiter. If the companion is $1 M_{\mathrm{Jup}}$, it would be located at $\sim 2$ AU. The constraints given by Equation (2) are also shown in Figure 7 (green dashed line). However, we stress that these assume that the companion has a circular orbit in the same plane as planets $b$ and $c$ and a mass much lower than that of the primary star. Therefore, it does not decrease the likelihood of close-in companions bright enough to significantly dilute the transit depth.

\section{INDIVIDUAL SYSTEMS}

Here we summarize some of the most important characteristics of each system determined from this study, as well as other studies where relevant.
$K 2-5$ is a $\sim \mathrm{K} 7.5 \mathrm{~V}$ star, hosting at least two planets with $P=5.7$ and 10.9 days and $R_{p}=1.91 \pm 0.44 R_{\oplus}$ and $2.26 \pm 0.62 R_{\oplus}$, respectively (Figure 8, Table 6). Both planets were reported by Foreman-Mackey et al. (2015) and Vanderburg et al. (2016) and validated by Montet et al. (2015b). The large uncertainties on planet radii $(\sim 20 \%-30 \%)$ relative to the rest of our targets are due to the modest $\mathrm{S} / \mathrm{N}$ of our IRTF-SpeX stellar spectrum (see Section 2.4.2).

$K 2-3$ is a nearby, $\sim \mathrm{M} 0 \mathrm{~V}$ star hosting three super-Earths with $P=10.1,24.6$, and 44.6 days and $R_{p}=2.18 \pm 0.30 R_{\oplus}$, $1.85 \pm 0.27 R_{\oplus}$, and $1.51 \pm 0.23 R_{\oplus}$, respectively (Figure 9, Table 7). All three planets were first reported in Crossfield et al. (2015). Spitzer observations independently confirmed transits of each planet (Beichman et al. 2016). Almenara et al. (2015) and Dai et al. (2016) measured masses of $8.4 \pm 2.1 M_{\oplus}$ and $8.1_{-1.9}^{+2.0} M_{\oplus}$, respectively, for the inner planet, $\mathrm{K} 2-3 \mathrm{~b}$, which suggests that it is composed mostly of rock, but possibly as much as $60 \%$ water. The outer planet receives $\sim 50 \%$ more stellar flux than Earth receives from the Sun, with an equilibrium temperature $T_{\mathrm{eq}} \sim 282 \mathrm{~K}$.

$K 2-8$ is a $\sim \mathrm{K} 3 \mathrm{~V}$ star hosting two planets with $P=5.1$ and 10.4 days, near a 2:1 MMR. These planets have radii of $2.41 \pm 0.33 R_{\oplus}$ and $3.58 \pm 0.71 R_{\oplus}$, respectively (Figure 10, Table 8). Both planets were reported in Foreman-Mackey et al. (2015) and Vanderburg et al. (2016). Montet et al. (2015b) estimated FPPs using VESPA. In their study, only the outer planet candidate (K2-8b) was given a "validated" disposition. They listed the inner planet as a "planet candidate" because the 

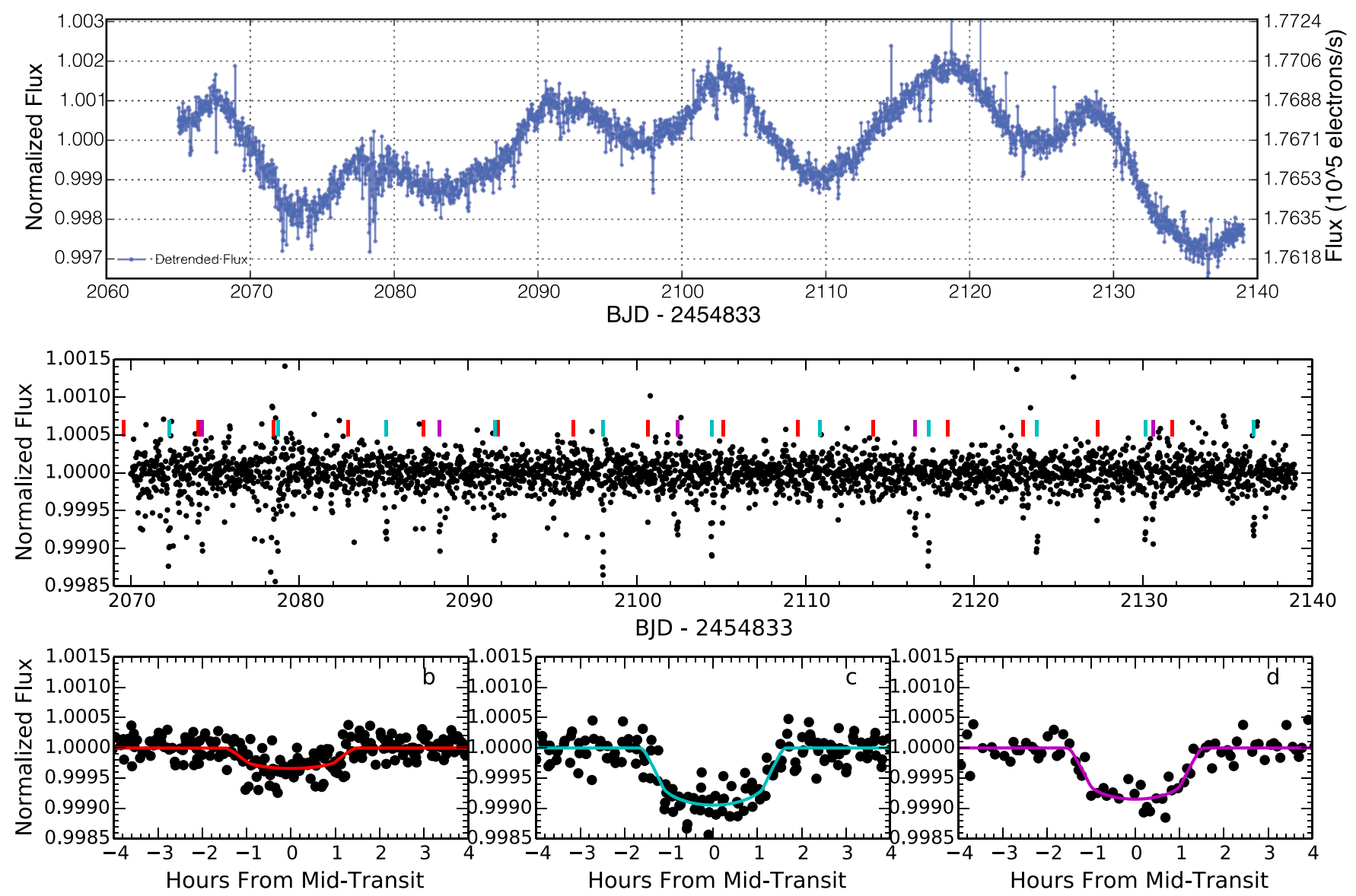

Figure 16. Top: $K 2$ photometry for K2-37 after subtracting variations caused by telescope roll. Middle: calibrated $K 2$ photometry. Vertical ticks indicate times of planet transits. Bottom: phase-folded photometry and best-fitting light curves for each planet.

FPP exceeded $1 \%$, dominated by the $1.9 \%$ probability of a background eclipsing binary (BEB). They looked for companions using images from Data Release 9 of the Sloan Digital Sky Survey (SDSS), which has a 1!"4 PSF (Ahn et al. 2012). Our NIRC2 AO imaging excludes the possibility of BEBs several times closer to the star, and we compute a lower FPP of $0.8 \%$. Surprisingly, for the outer planet candidate validated by Montet et al. (2015b) (FPP $=0.2 \%)$, we compute a higher FPP of $1.3 \%$, despite better constraints from AO imaging and spectroscopy. The reason for this discrepancy is unclear but possibly the result of having slightly different stellar parameter constraints or photometry. Nevertheless, VESPA does not account for the multiplicity of this system, which would reduce our FPP estimate below 1\% (See Section 4.4). Therefore, we assign validated dispositions to both $\mathrm{K} 2-8 \mathrm{~b}$ and $\mathrm{K} 2-8 \mathrm{c}$.

$K 2-19$ is a $\sim \mathrm{G} 9 \mathrm{~V}$ star hosting three planets. The star is magnetically active; we measure $\log R_{\mathrm{HK}}^{\prime}=-4.66 \mathrm{dex}$, and the light curve exhibits quasi-periodic variations in brightness by $\sim 1 \%$ over 15-20 days. The inner planet, with a period of $P=2.5$ days, is near Earth size with $R_{p}=1.14 \pm 0.13 R_{\oplus}$. The outer two planets are larger and near 3:2 MMR, having $P=7.9$ and 11.9 days and $R_{p}=7.74 \pm 0.39 R_{\oplus}$ and $4.86_{-0.44}^{+0.62} R_{\oplus}$, respectively (Figure 11, Table 9). The two outer planets were reported as planet candidates by Foreman-Mackey et al. (2015) and first confirmed by Armstrong et al. (2015), who used ground-based telescopes to detect additional transits and measure hour-long TTVs for the middle planet. A similar study by Narita et al. (2015) found consistent TTVs and precisely characterized the star via AO imaging with SubaruHiCIAO and high-resolution spectroscopy with Subaru-HDS. Barros et al. (2015) simultaneously modeled $K 2$ and groundbased photometry and RVs from the SOPHIE spectrograph of $\sim 16 \mathrm{~m} \mathrm{~s}^{-1}$ precision. They find that planet $\mathrm{b}$ has mass $44 \pm 12$ $M_{\oplus}$ and radius $7.46 \pm 0.76 R_{\oplus}$, while planet c has mass $15.9_{-2.8}^{+7.7} M_{\oplus}$ and radius $4.51 \pm 0.47 R_{\oplus}$. Dai et al. (2016) measured $M_{b}=28.5_{-5.0}^{+5.4} M_{\oplus}$ and $M_{c}=25.6 \pm 7.1 M_{\oplus}$. Planet d was first reported as a planet candidate by Vanderburg et al. (2016). The 2.5-day period of the transits is $\sim 10 \times$ the spacecraft thruster firing period of $6 \mathrm{hr}$. However, in our planet search and light-curve fitting, we omit all photometry collected during a thruster firing. Thus, we are confident that thruster firings are not the source of the transit. As an additional test, we visually inspected the complete set of photometric measurements phased at the transit period and found that there was no excess of thruster firings during the transits. Thus, even if these data points were included in our fitting, they would not significantly bias the derived planet properties. We also verified that the photometric scatter during the transit phase is not systematically different than the out-oftransit phase.

$K 2-35$ is a bright $\sim \mathrm{K} 4 \mathrm{~V}$ star hosting two close-in superEarths with $P=2.4$ and 5.6 days. The planets are $1.40 \pm$ $0.17 R_{\oplus}$ and $2.09_{-0.24}^{+0.33} R_{\oplus}$, respectively (Figure 12 , Table 10 ). The outer planet was reported as a planet candidate by Foreman-Mackey et al. (2015) and Montet et al. (2015b). Both were listed as planet candidates by Vanderburg et al. (2016). 

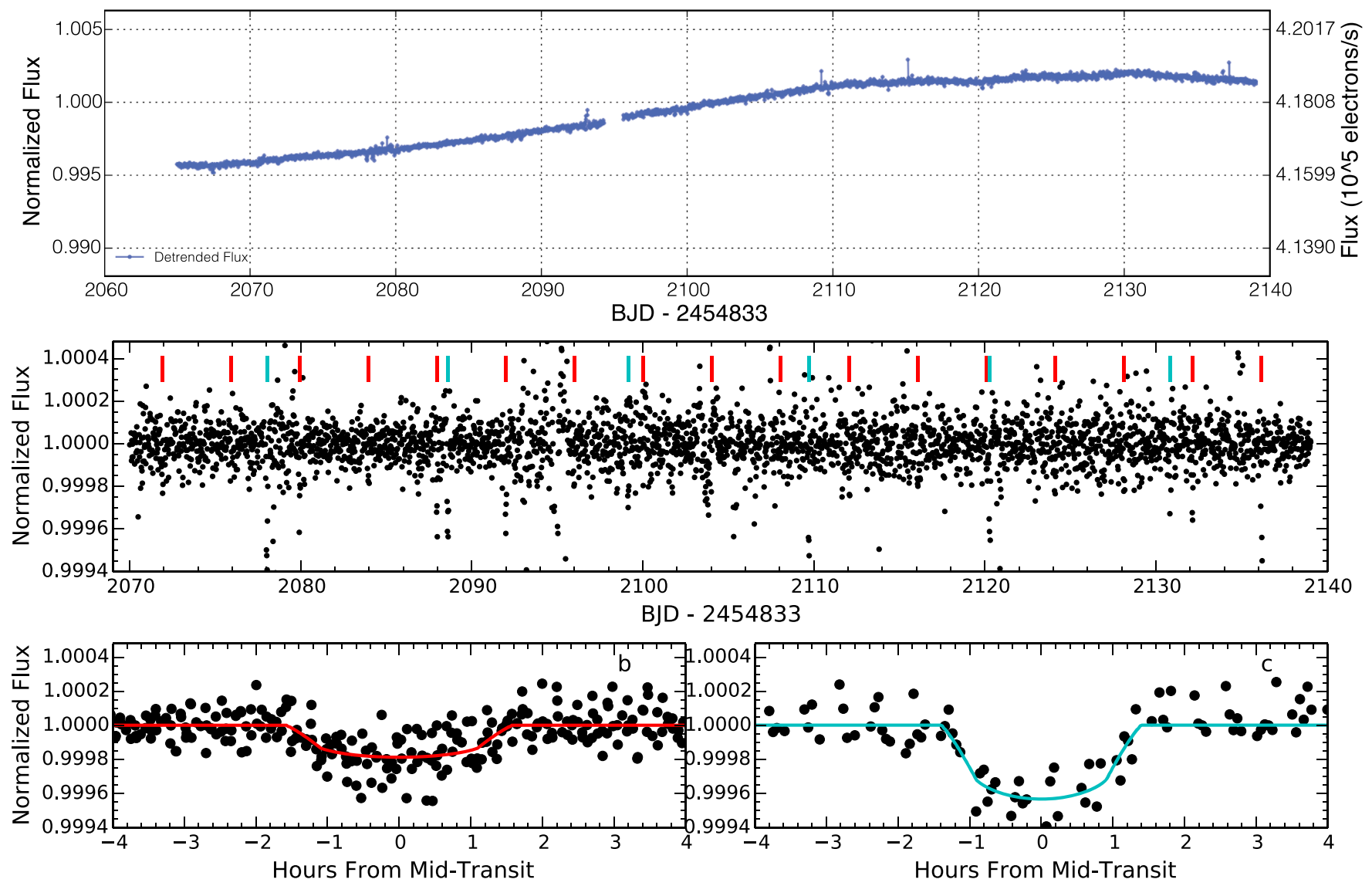

Figure 17. Top: $K 2$ photometry for K2-38 after subtracting variations caused by telescope roll. Middle: calibrated $K 2$ photometry. Vertical ticks indicate times of planet transits. Bottom: phase-folded photometry and best-fitting light curves for each planet.

$K 2-36$ is a bright $\sim \mathrm{K} 2 \mathrm{~V}$ star hosting two hot super-Earths with $P=1.4$ and 5.3 days, first reported as planet candidates by Vanderburg et al. (2016). The inner planet, $R_{p}=1.32 \pm$ $0.09 R_{\oplus}$, has $T_{\mathrm{eq}} \sim 1200 \mathrm{~K}$, and the outer planet, $R_{p}=$ $2.80_{-0.31}^{+0.43} R_{\oplus}$, has $T_{\text {eq }} \sim 800 \mathrm{~K}$ (Figure 13, Table 11). The light curve shows $1 \%-2 \%$ modulation with a period of $\sim 10$ days, and the spectrum has strong $\mathrm{Ca}$ II $\mathrm{H}$ and $\mathrm{K}$ emission lines $\left(S_{\mathrm{HK}}=0.46\right)$, indicating that the star is magnetically active.

$K 2-16$ is a faint, $\sim \mathrm{K} 3 \mathrm{~V}$ star having two planets with $P=7.6$ and 19.1 days and $R_{p}=2.02 \pm 0.24 R_{\oplus}$ and $2.54_{-0.47}^{+1.12} R_{\oplus}$, respectively (Figure 14, Table 12). These planets are near 5:2 MMR. They were first detected by Foreman-Mackey et al. (2015) and validated by Montet et al. (2015b). Both planets were detected by Vanderburg et al. (2016).

$K 2-24$ is a bright $\sim \mathrm{G} 9 \mathrm{~V}$ star with two cool, sub-Saturn-size planets near 2:1 MMR, with $P=20.9$ and 42.4 days and $R_{p}=5.83 \pm 0.60 R_{\oplus}$ and $8.10 \pm 0.82 R_{\oplus}$, respectively (Figure 15, Table 13). Using Keck/HIRES RVs, Petigura et al. (2016) measured masses of $21 \pm 5.4 M_{\oplus}$ and $27 \pm 6.9$ $M_{\oplus}$ and densities of $0.63 \pm 0.25 \mathrm{~g} \mathrm{~cm}^{-3}$ and $0.31 \pm$ $0.12 \mathrm{~g} \mathrm{~cm}^{-3}$ for inner and outer planets, respectively. Dai et al. (2016) measured masses of $19.8_{-4.4}^{+4.5} M_{\oplus}$ and 26.0 $0_{-6.1}^{+5.8} M_{\oplus}$, respectively. The transit signals of both planets were also detected by Vanderburg et al. (2016).

$K 2-37$ is a bright $\sim \mathrm{G} 3 \mathrm{~V}$ star with three small, tightly packed planets having $P=9.0,24.6$, and 44.6 days and $R_{p}=$ $1.61 \pm 0.17, \quad R_{\oplus} \quad 2.75 \pm 0.27 R_{\oplus}, \quad$ and $2.73 \pm 0.36 R_{\oplus}$, respectively (Figure 16, Table 14). These were reported as planet candidates by Vanderburg et al. (2016).

$K 2-38$ is a bright $\sim \mathrm{G} 2 \mathrm{~V}$ star, with two hot super-Earths, with $P=4.0$ and 10.6 days and $R_{p}=1.55 \pm 0.16 R_{\oplus}$ and $2.42 \pm 0.29 R_{\oplus}$, respectively (Figure 17, Table 15). We measure planet masses of $12.0 \pm 2.9 M_{\oplus}$ and $9.9 \pm 4.6 M_{\oplus}$ and densities of $17.5_{-6.2}^{+8.5} \mathrm{~g} \mathrm{~cm}^{-3}$ and $3.6_{-1.9}^{+2.7} \mathrm{~g} \mathrm{~cm}^{-3}$, respectively (Table 5). These indicate that the inner planet is likely rocky and possibly iron-rich, while the outer planet is likely to have an envelope of low-density volatiles (Section 8.1). A linear RV trend also suggests a third companion at larger orbital distances (Section 6). None of these planets were previously reported.

$K 2-32$ is a bright $\sim$ G9V star with three planets, with $P=9.0$, 24.6 , and 44.6 days and $R_{p}=5.38 \pm 0.35, R_{\oplus} 3.48_{-0.42}^{+0.97}, R_{\oplus}$ and $3.75 \pm 0.40 R_{\oplus}$, respectively (Figure 18, Table 16). Vanderburg et al. (2016) reported these as planet candidates. The outer two planets are near 3:2 MMR. Dai et al. (2016) confirmed the inner planet $\mathrm{b}$, using RVs to measure a mass of $21.1 \pm 5.9 M_{\oplus}$. For planet c, VESPA returns an FPP of $2.2 \%$, which does not meet our criterion for a "validated" disposition (FPP $<1 \%$ ). However, VESPA does not account for the "multiplicity boost," which is more than the factor of 2.2 necessary to reduce the FPP of K2-32c below $1 \%$ (see discussion in Section 4.4). Therefore, we deem all three planet candidates in this system "validated." 

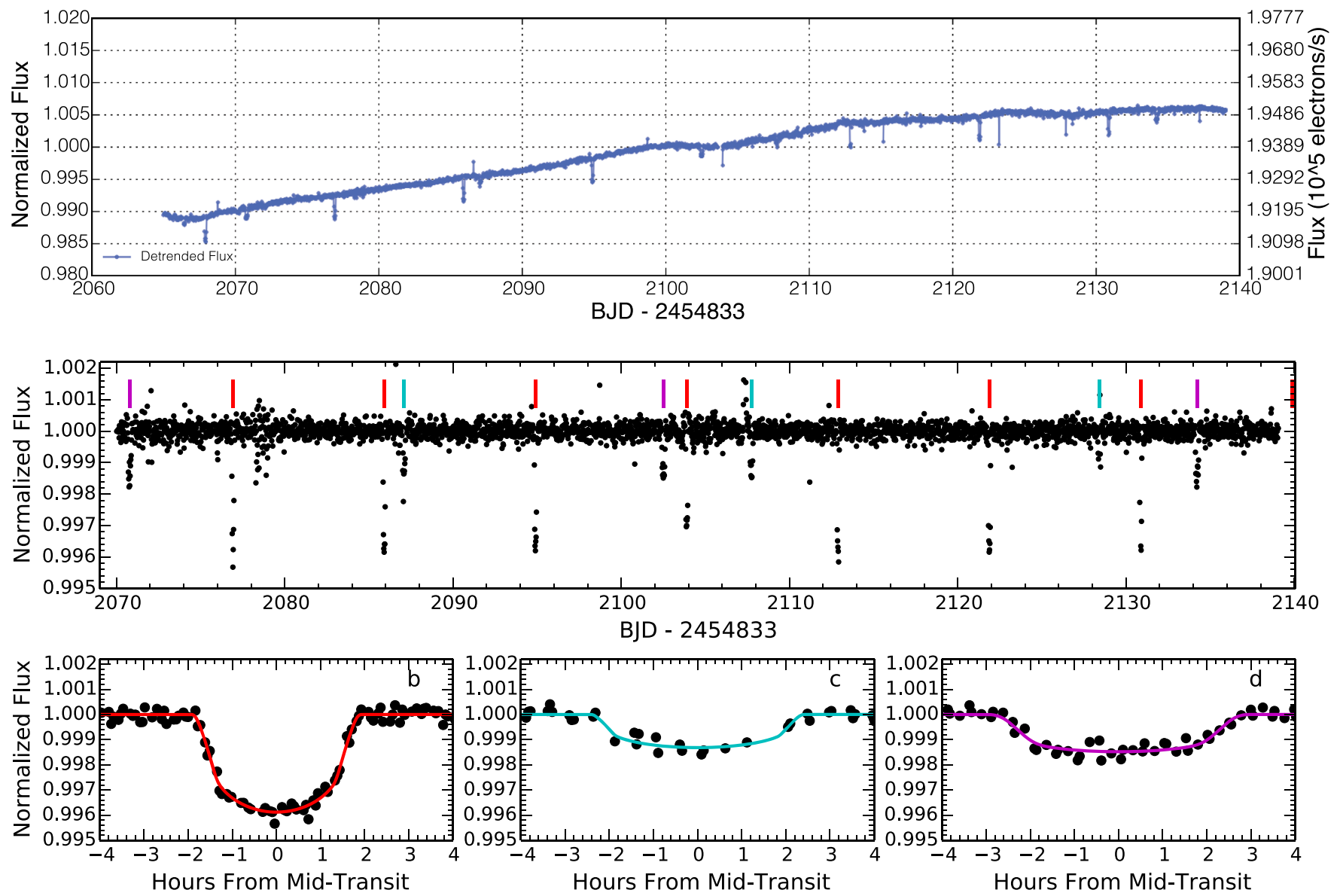

Figure 18. Top: $K 2$ photometry for K2-32 after subtracting variations caused by telescope roll. Middle: calibrated $K 2$ photometry. Vertical ticks indicate times of planet transits. Bottom: phase-folded photometry and best-fitting light curves for each planet.

\section{DISCUSSION AND CONCLUSIONS}

We have detected, validated, and characterized 11 multiplanet systems composed of 26 planets in $K 2$ fields $\mathrm{C} 1$ and $\mathrm{C} 2$. Seven of these systems have two detected planets and four of them have three detected planets, the majority of which are smaller than Neptune. Moreover, seven of the stars have $K p<13$ and are amenable to RV follow-up to measure planet masses and densities. This study is distinguished from previous $K 2$ catalogs because it focuses on multiplanet systems with intrinsically low FPPs and we have characterized each host star with high-contrast imaging and spectroscopy. We detected the $\mathrm{RV}$ signatures of K2-38b and K2-38c, allowing us to constrain their masses and densities and infer their bulk compositions.

\subsection{Compositions of K2-38 Super-Earths}

Figure 19 shows the mass-radius and density-radius distributions of all planets with $R_{p}<4.0 R_{\oplus}$ whose mass and radius are measured to better than $50 \%$ precision $(2 \sigma)$ by either RVs or TTVs. ${ }^{22}$ Solar system planets are included, as well as theoretical mass-radius relations for pure iron, rock, and water compositions, based on models by Zeng \& Sasselov (2013). The red points in Figure 19 show our mass and radius constraints of K2-38b and K2-38c.

\footnotetext{
22 NASA Exoplanet Archive, UT 2015 November 13, http:// exoplanetarchive.ipac.caltech.edu.
}

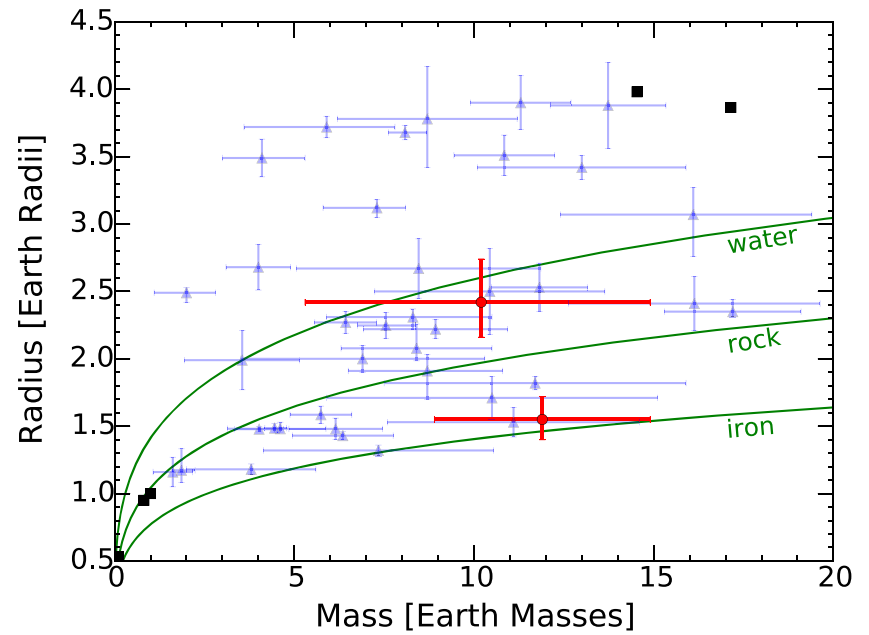

Figure 19. Radii and masses of all confirmed planets whose mass and radius are measured to better than $50 \%(2 \sigma)$ precision (blue triangles). Solar system planets are represented as black squares. Red circles indicate our measurements of K2-38b and K2-38c (top and bottom points, respectively). Green curves show the expected planet mass-radius curves for pure iron, rock, and water compositions according to models by Zeng \& Sasselov (2013). K2-38b likely has a large iron fraction and could be the densest planet detected to date. The composition of $\mathrm{K} 2-38 \mathrm{c}$ is less certain, but the planet likely possesses an outer envelope composed of low-density volatiles. 


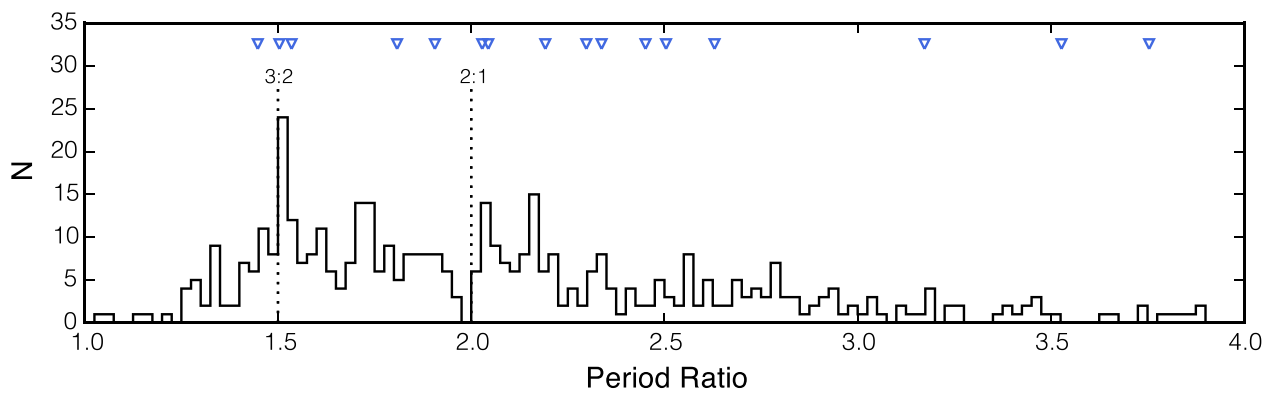

Figure 20. Histogram of the distribution of period ratios for planets from the prime Kepler mission (Fabrycky et al. 2014). In systems with three or more transiting planets, all pairs of planets are considered, not only adjacent pairs. In order to make a more direct comparison to the population probed by $K 2$, we have only shown Kepler planets having $P<50$ days. The period ratios of $K 2$ planet pairs presented in this work are shown using blue triangles. The $K 2$ distribution of period ratios is qualitatively similar to the period ratios from the Kepler prime mission.

Table 4

Relative Radial Velocities, K2-38

\begin{tabular}{lcc}
\hline \hline BJD-2,457,000 & $\begin{array}{c}\text { Radial Velocity } \\
\left(\mathrm{m} \mathrm{s}^{-1}\right)\end{array}$ & $\begin{array}{c}\text { Uncertainty } \\
\left(\mathrm{m} \mathrm{s}^{-1}\right)\end{array}$ \\
\hline 197.81009 & 6.49 & 1.49 \\
200.88524 & 6.96 & 1.42 \\
206.87767 & -3.70 & 1.36 \\
207.86695 & -4.20 & 1.46 \\
208.87926 & 5.25 & 1.40 \\
210.87654 & -6.06 & 1.34 \\
215.91724 & 2.97 & 1.46 \\
236.80323 & 4.93 & 1.40 \\
245.80773 & 3.87 & 1.26 \\
255.77713 & -0.10 & 1.44 \\
262.77317 & -7.03 & 1.51 \\
265.74243 & -1.96 & 1.27 \\
290.74044 & -8.97 & 1.75 \\
298.71937 & -3.67 & 1.76 \\
\hline
\end{tabular}

Table 5

RV Model, K2-38

\begin{tabular}{lccll}
\hline \hline Parameter & Planet b & & Planet c & Units \\
\hline$K$ & $4.6 \pm 1.1$ & & $2.8 \pm 1.3$ & $\mathrm{~m} \mathrm{~s}^{-1}$ \\
$M_{p}$ & $12.0 \pm 2.9$ & & $9.9 \pm 4.6$ & $M_{\oplus}$ \\
$\rho_{p}$ & $17.5_{-6.2}^{+8.5}$ & & $3.6_{-1.9}^{+2.7}$ & $\mathrm{~g} \mathrm{~cm}^{-3}$ \\
$\sigma_{\text {jit }}$ & & $2.4_{-0.7}^{+1.0}$ & & $\mathrm{~m} \mathrm{~s}^{-1}$ \\
$\gamma$ & & $-1.7 \pm 0.9$ & & $\mathrm{~m} \mathrm{~s}^{-1}$ \\
$d v / d t$ & & $-37 \pm 11$ & & $\mathrm{~m} \mathrm{~s}^{-1} \mathrm{yr}^{-1}$ \\
\hline
\end{tabular}

Note. $K-\mathrm{RV}$ semi-amplitude; $M_{p}$ —planet mass; $\rho_{p}$-planet density; $\sigma_{\mathrm{jit}}-\mathrm{RV}$ "jitter"; $\gamma$-constant RV offset; $d v / d t$-constant RV acceleration.

The measured mass and radius of $\mathrm{K} 2-38 \mathrm{~b}$ are consistent with a rocky or iron-rich composition, matching K2-3d and KOI$94 \mathrm{~b}$ within uncertainties. Comparing mass and radius estimates with compositional models of Zeng \& Sasselov (2013) gives a $97.7 \%$ probability that the planet is denser than pure rock. With a bulk density of $17.5_{-6.2}^{+8.5} \mathrm{~g} \mathrm{~cm}^{-3}, \mathrm{~K} 2-38 \mathrm{~b}$ could be the densest planet discovered to date, but additional RV measurements are needed to confirm this. While our $1 \sigma$ measurement errors do not rule out densities exceeding that of pure iron, we can reject such compositions on the basis that they are incompatible with planet formation theory and the low abundances of heavy
Table 6

Planet Properties, K2-5

\begin{tabular}{|c|c|c|c|}
\hline Parameter & Planet b & Planet c & Units \\
\hline \multicolumn{4}{|c|}{ Transit Model } \\
\hline$T_{0}$ & $808.8600 \pm 0.0048$ & $814.6010 \pm 0.0052$ & $\begin{array}{r}\mathrm{BJD}_{\mathrm{TDB}}- \\
2,456,000\end{array}$ \\
\hline$P$ & $5.73594 \pm 0.00064$ & $10.93241 \pm 0.00134$ & days \\
\hline$i$ & $87.35_{-3.37}^{+1.88}$ & $86.95 \pm 2.47$ & deg \\
\hline$R_{p} / R_{\star}$ & $0.03031_{-0.00236}^{+0.00412}$ & $0.03601_{-0.00475}^{+0.00805}$ & $\cdots$ \\
\hline$R_{\star} / a$ & $0.0712_{-0.0159}^{+0.0487}$ & $0.0639_{-0.0272}^{+0.0448}$ & $\cdots$ \\
\hline$u$ & $0.62 \pm 0.05$ & $0.62 \pm 0.05$ & $\cdots$ \\
\hline$b$ & $0.65_{-0.41}^{+0.22}$ & $0.83_{-0.43}^{+0.09}$ & $\ldots$ \\
\hline$t_{14}$ & $2.654_{-0.268}^{+0.607}$ & $3.498_{-0.580}^{+1.420}$ & $\mathrm{hr}$ \\
\hline$R_{p}$ & $1.91 \pm 0.44$ & $2.26 \pm 0.62$ & $R_{\oplus}$ \\
\hline$\rho_{\star, \mathrm{circ}}$ & $1.59_{-1.25}^{+1.80}$ & $0.60_{-0.48}^{+2.59}$ & $\mathrm{~g} \mathrm{~cm}^{-3}$ \\
\hline \multicolumn{4}{|c|}{ Derived Properties } \\
\hline$a$ & $0.0532 \pm 0.0038$ & $0.0818 \pm 0.0059$ & $\mathrm{AU}$ \\
\hline$S_{\mathrm{inc}}$ & $24.1_{-11.3}^{+18.4}$ & $10.2_{-4.8}^{+7.8}$ & $S_{\oplus}$ \\
\hline$T_{\text {eq }}$ & $565 \pm 84$ & $456 \pm 68$ & $\mathrm{~K}$ \\
\hline
\end{tabular}

Note. $T_{0}$-mid-transit time; $i$-orbital inclination; $a$-orbital semimajor axis; $u$ -linear limb-darkening coefficient; $b$-impact parameter; $t_{14}$-transit duration; $\rho_{\star, \text { circ }}$-stellar density; $S_{\text {inc }}$-incident stellar flux; $T_{\text {eq }}$ equilibrium temperature.

elements in planet-forming disks. Iron-rich planets (e.g., Mercury) might result from collisional stripping of the rocky mantle of a larger, differentiated planet. Simulations by Marcus et al. (2010) suggest that collisional stripping is unlikely to produce super-Earths with iron mass fractions exceeding $\sim 70 \%$. The initial assembly of an iron-rich core might be expedited by photophoretic segregation of metals and silicates in the inner protoplanetary disk, which preferentially drives the rocky material outward (Wurm et al. 2013). With an equilibrium temperature $T_{\mathrm{eq}} \sim 1200 \mathrm{~K}, \mathrm{~K} 2-38 \mathrm{~b}$ is perhaps a remnant core of a larger planet whose atmosphere was removed by photoevaporation. In such a scenario, the precursor could have been a gas giant that formed beyond the snow line and migrated inward. Indeed, such a massive core would have rapidly accreted nebular gas, if still present. Alternatively, if the planet assembled in situ, photoevaporation might have been less important to its present composition; nebular gas might have dispersed before the core was massive enough to accrete, or atmospheric accretion could have been limited by the creation of a gap in the disk (Hansen \& Murray 2012). 
Table 7

Planet Properties, K2-3

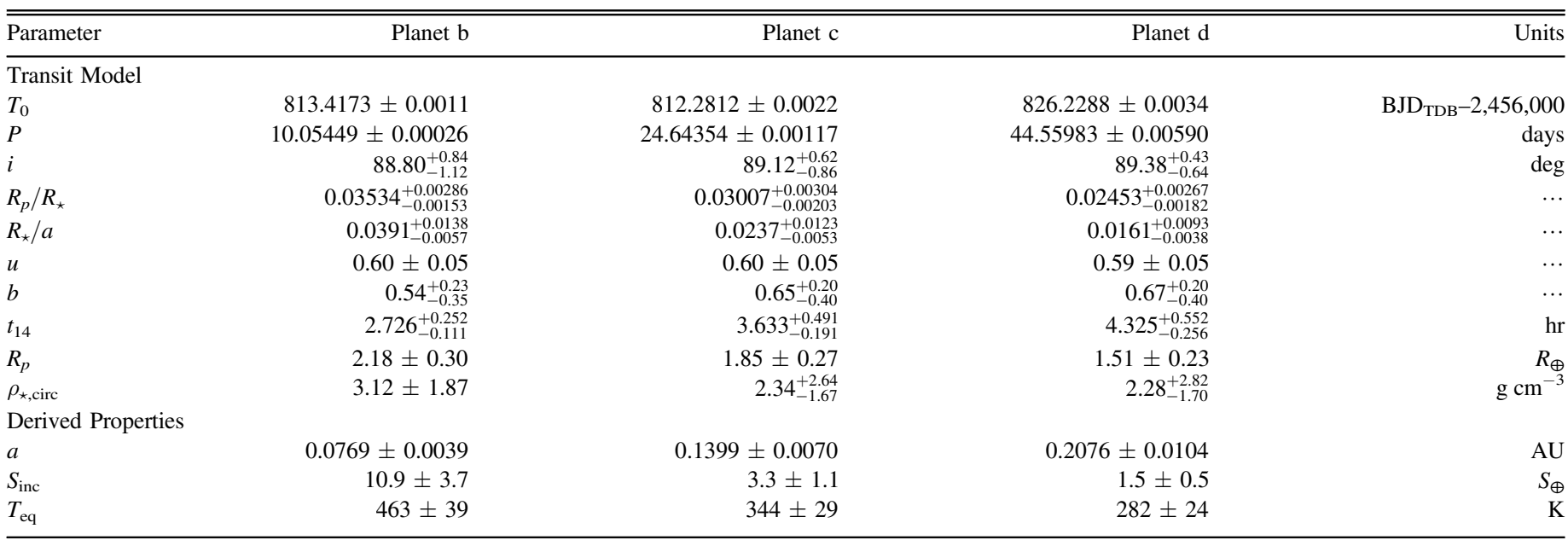

Note. Same footnotes as Table 6.

Table 8

Planet Properties, K2-8

\begin{tabular}{|c|c|c|c|}
\hline Parameter & Planet $b$ & Planet c & Units \\
\hline \multicolumn{4}{|c|}{ Transit Model } \\
\hline$T_{0}$ & $813.6114 \pm 0.0030$ & $813.0707 \pm 0.0033$ & $\begin{array}{l}\mathrm{BJD}_{\mathrm{TDB}}- \\
2,456,000\end{array}$ \\
\hline$P$ & $10.35239 \pm 0.00086$ & $5.06416 \pm 0.00041$ & days \\
\hline$i$ & $86.42_{-1.37}^{+2.56}$ & $86.70 \pm 2.48$ & deg \\
\hline$R_{p} / R_{\star}$ & $0.04457 \pm 0.00861$ & $0.02990 \pm 0.00385$ & $\cdots$ \\
\hline$R_{\star} / a$ & $0.0710_{-0.0366}^{+0.0233}$ & $0.0743_{-0.0251}^{+0.0415}$ & $\cdots$ \\
\hline$u$ & $0.70 \pm 0.05$ & $0.70 \pm 0.05$ & $\cdots$ \\
\hline$b$ & $0.88_{-0.36}^{+0.04}$ & $0.78_{-0.42}^{+0.12}$ & $\cdots$ \\
\hline$t_{14}$ & $3.362 \pm 0.885$ & $2.080_{-0.253}^{+0.498}$ & $\mathrm{hr}$ \\
\hline$R_{p}$ & $3.58 \pm 0.71$ & $2.41 \pm 0.33$ & $R_{\oplus}$ \\
\hline$\rho_{\star, \operatorname{circ}}$ & $0.49_{-0.28}^{+3.85}$ & $1.79_{-1.32}^{+4.39}$ & $\mathrm{~g} \mathrm{~cm}^{-3}$ \\
\hline \multicolumn{4}{|c|}{ Derived Properties } \\
\hline$a$ & $0.0856 \pm 0.0014$ & $0.0532 \pm 0.0009$ & $\mathrm{AU}$ \\
\hline$S_{\text {inc }}$ & $37.7 \pm 4.4$ & $97.8 \pm 11.4$ & $S_{\oplus}$ \\
\hline$T_{\mathrm{eq}}$ & $631 \pm 18$ & $801 \pm 23$ & K \\
\hline
\end{tabular}

Note. Same footnotes as Table 6 .

The mass and radius of the outer planet, K2-38c, are consistent with many other planets, including GJ 1214b, Kepler-68b, Kepler-96b, Kepler-102e, Kepler-106c, HD $97658 \mathrm{~b}$, and HIP 116454b. Its equilibrium temperature of $858 \pm 37 \mathrm{~K}$ is intermediate to those of the other planets, which have $T_{\text {eq }}$ spanning $\sim 550-1150 \mathrm{~K}$. This planet is unlikely to have experienced significant atmospheric photoevaporation. We measure the planet's mass to $\sim 50 \%(2 \sigma)$ precision, which allows for a range of possible compositions-even with smaller measurement uncertainties, planet compositions in this region of the mass-radius diagram are highly degenerate (Seager et al. 2007; Adams et al. 2008; Valencia et al. 2013). K2-38c likely contains an outer envelope composed of low-density volatiles. It could have a small rocky core, with an extended $\mathrm{H}$ / He envelope or steam atmosphere. Alternatively, since the measured density is consistent with pure water, the planet could be a "water-world," with a core rich in water ice and interior to a mostly steam atmosphere. A more precise mass is needed to meaningfully constrain core-to-envelope mass ratios and possible mass fractions of rock, water, and $\mathrm{H} / \mathrm{He}$. Due to the mass-radius degeneracies between water-worlds and rocky cores with extended $\mathrm{H} / \mathrm{He}$ atmospheres, the atmospheric composition must be measured by other means (e.g., transmission spectroscopy) in order to distinguish between these two different archetypes.

\subsection{Orbital Stability}

We analytically assess the orbital stability of each system by comparing orbital separations of each planet pair to their mutual Hill radii, which is the length scale applicable to dynamical interactions:

$$
R_{H}=\left[\frac{M_{\text {in }}+M_{\text {out }}}{3 M_{\star}}\right]^{1 / 3} \frac{\left(a_{\text {in }}+a_{\text {out }}\right)}{2} .
$$

Here $M_{\text {in }}$ and $M_{\text {out }}$ are the masses of the inner and outer planets and $a_{\text {in }}$ and $a_{\text {out }}$ are their respective orbital distances from the host star. For planets with $R_{p}=1.4-4.0 R_{\oplus}$, we use the powerlaw scaling $M_{p}=2.69 R_{p}^{0.93}$ from Weiss \& Marcy (2014) to convert radii to masses. For planets with $R_{p}>4.0 R_{\oplus}$, we use $M_{p}=1.6 R_{p}^{1.8}$ (Wolfgang et al. 2015). The one exception is $\mathrm{K} 2-$ 38 , for which we use our measured RV masses (Section 6). We compute orbital separations in units of $R_{H}$ :

$$
\Delta=\frac{a_{\text {out }}-a_{\text {in }}}{R_{H}} .
$$

For a two-planet system, if $\Delta<2 \sqrt{3}$, then even circular orbits are likely to be unstable on short timescales (Gladman 1993). All of our $K 2$ multis have $\Delta>5$, so we have no reason to suspect that their orbits are unstable. While there are no such analytic criteria to assess the orbital stability of threeplanet systems, Fabrycky et al. (2014) suggest $\Delta_{\text {in }}+\Delta_{\text {out }}>18$ as a conservative requirement. All four of the triple-planet systems presented here satisfy this criterion. 
Table 9

Planet Properties, K2-19

\begin{tabular}{|c|c|c|c|c|}
\hline Parameter & Planet $b$ & Planet c & Planet d & Units \\
\hline \multicolumn{5}{|c|}{ Transit Model } \\
\hline$T_{0}$ & $813.3837 \pm 0.0003$ & $817.2755 \pm 0.0051$ & $808.9207 \pm 0.0086$ & $\mathrm{BJD}_{\mathrm{TDB}}-2,456,000$ \\
\hline$P$ & $7.91940 \pm 0.00005$ & $11.90715 \pm 0.00150$ & $2.50856 \pm 0.00041$ & days \\
\hline$R_{p} / R_{\star}$ & $0.07540_{-0.00043}^{+0.00060}$ & $0.04727_{-0.00352}^{+0.00568}$ & $0.01109 \pm 0.00116$ & $\cdots$ \\
\hline$R_{\star} / a$ & $0.0540_{-0.0010}^{+0.0021}$ & $0.0553_{-0.0121}^{+0.0287}$ & $0.1277_{-0.0254}^{+0.0586}$ & .. \\
\hline$u$ & $0.48 \pm 0.03$ & $0.64 \pm 0.05$ & $0.64 \pm 0.05$ & $\cdots$ \\
\hline$R_{p}$ & $7.74 \pm 0.39$ & $4.86_{-0.44}^{+0.62}$ & $1.14 \pm 0.13$ & $R_{\oplus}$ \\
\hline$\rho_{\star, \text { circ }}$ & $1.91_{-0.21}^{+0.12}$ & $0.79_{-0.56}^{+0.87}$ & $1.44_{-0.97}^{+1.36}$ & $\mathrm{~g} \mathrm{~cm}^{-3}$ \\
\hline \multicolumn{5}{|c|}{ Derived Properties } \\
\hline$a$ & $0.0740 \pm 0.0012$ & $0.0971 \pm 0.0016$ & $0.0344 \pm 0.0006$ & $\mathrm{AU}$ \\
\hline$S_{\text {inc }}$ & $125.9 \pm 14.4$ & $73.1 \pm 8.4$ & $583.5 \pm 66.7$ & $S_{\oplus}$ \\
\hline
\end{tabular}

Note. Same footnotes as Table 6

Table 10

Planet Properties, K2-35

\begin{tabular}{|c|c|c|c|}
\hline Parameter & Planet b & Planet c & Units \\
\hline \multicolumn{4}{|c|}{ Transit Model } \\
\hline$T_{0}$ & $810.5871 \pm 0.0085$ & $812.1158 \pm 0.0049$ & $\begin{array}{c}\mathrm{BJD}_{\mathrm{TDB}}- \\
2,456,000\end{array}$ \\
\hline$P$ & $2.39984 \pm 0.00039$ & $5.60912 \pm 0.00071$ & days \\
\hline$i$ & $86.10_{-4.43}^{+2.67}$ & $87.85_{-2.25}^{+1.51}$ & deg \\
\hline$R_{p} / R_{\star}$ & $0.01777_{-0.00166}^{+0.00234}$ & $0.02661_{-0.00266}^{+0.00407}$ & \\
\hline$R_{\star} / a$ & $0.1237_{-0.0230}^{+0.0539}$ & $0.0575_{-0.0133}^{+0.0319}$ & \\
\hline$u$ & $0.72 \pm 0.05$ & $0.72 \pm 0.05$ & \\
\hline$b$ & $0.56_{-0.35}^{+0.26}$ & $0.66_{-0.40}^{+0.20}$ & . \\
\hline$t_{14}$ & $2.064 \pm 0.308$ & $2.050_{-0.227}^{+0.343}$ & hr \\
\hline$R_{p}$ & $1.40 \pm 0.17$ & $2.09_{-0.24}^{+0.33}$ & $R_{\oplus}$ \\
\hline$\rho_{\star, \mathrm{circ}}$ & $1.73 \pm 1.31$ & $3.16_{-2.32}^{+3.79}$ & $\mathrm{~g} \mathrm{~cm}^{-3}$ \\
\hline \multicolumn{4}{|c|}{ Derived Properties } \\
\hline$a$ & $0.0320 \pm 0.0005$ & $0.0564 \pm 0.0009$ & $\mathrm{AU}$ \\
\hline$S_{\text {inc }}$ & $217.4 \pm 25.5$ & $70.1 \pm 8.2$ & $S_{\oplus}$ \\
\hline$T_{\text {eq }}$ & $979 \pm 29$ & $737 \pm 22$ & $\mathrm{~K}$ \\
\hline
\end{tabular}

Note. Same footnotes as Table 6.

\subsection{Orbital Resonances}

In multiplanet systems, the distribution of planet orbital period ratios contains important clues regarding their formation and evolution. Fabrycky et al. (2014) found that the distribution of period ratios among Kepler multis was fairly uniform. They noted, however, a slight overabundance of planet pairs just outside first-order MMRs and an underabundance of pairs just inside. Lithwick \& Wu (2012) and Batygin \& Morbidelli (2013) interpreted this feature as a natural outcome of resonant pairs of planets that experience eccentricity damping. Figure 20 shows the distribution of period ratios for planet pairs discovered during the prime Kepler mission. In order to make a more direct comparison to our $K 2$ planets, we have restricted
Table 11

Planet Properties, K2-36

\begin{tabular}{|c|c|c|c|}
\hline Parameter & Planet $\mathrm{b}$ & Planet c & Units \\
\hline \multicolumn{4}{|c|}{ Transit Model } \\
\hline$T_{0}$ & $809.4684 \pm 0.0017$ & $812.8422 \pm 0.0008$ & $\begin{array}{l}\mathrm{BJD}_{\mathrm{TDB}} \\
2,456,000\end{array}$ \\
\hline$P$ & $1.42266 \pm 0.00005$ & $5.34059 \pm 0.00010$ & days \\
\hline$i$ & $87.75_{-2.40}^{+1.62}$ & $88.33_{-1.63}^{+1.19}$ & deg \\
\hline$R_{p} / R_{\star}$ & $0.01625_{-0.00060}^{+0.00093}$ & $0.03468_{-0.00362}^{+0.00515}$ & ․ \\
\hline$R_{\star} / a$ & $0.1124_{-0.0091}^{+0.0201}$ & $0.0405_{-0.0117}^{+0.0250}$ & $\cdots$ \\
\hline$u$ & $0.69 \pm 0.05$ & $0.70 \pm 0.05$ & .. \\
\hline$b$ & $0.36 \pm 0.26$ & $0.72_{-0.42}^{+0.16}$ & ... \\
\hline$t_{14}$ & $1.206 \pm 0.078$ & $1.267_{-0.104}^{+0.301}$ & $\mathrm{hr}$ \\
\hline$R_{p}$ & $1.32 \pm 0.09$ & $2.80_{-0.31}^{+0.43}$ & $R_{\oplus}$ \\
\hline$\rho_{\star, \operatorname{circ}}$ & $6.57_{-2.56}^{+1.89}$ & $9.96_{-7.60}^{+17.74}$ & $\mathrm{~g} \mathrm{~cm}^{-3}$ \\
\hline \multicolumn{4}{|c|}{ Derived Properties } \\
\hline$a$ & $0.0230 \pm 0.0004$ & $0.0555 \pm 0.0009$ & $\mathrm{AU}$ \\
\hline$S_{\text {inc }}$ & $546.3 \pm 63.5$ & $93.6 \pm 10.9$ & $S_{\oplus}$ \\
\hline$T_{\text {eq }}$ & $1232 \pm 36$ & $793 \pm 23$ & $\mathrm{~K}$ \\
\hline
\end{tabular}

Note. Same footnotes as Table 6 .

to orbital periods $<50$ days. We have indicated the period ratios for the planet pairs presented in this paper. While there are too few planet pairs for a detailed comparison, the distribution of period ratios is qualitatively similar: fairly uniform, with a few planet pairs lying just outside the 2:1 and 3:2 MMRs.

Two planet pairs, K2-19bc and K2-32cd, are just wide of the 3:2 MMR, having $P_{\text {rel }}=1.5036$ and 1.5351 days, respectively. Two pairs, K2-24bc and K2-8bc, orbit just outside of 2:1 resonance $\left(P_{\text {rel }}=2.0284\right.$ and 2.0441 days $)$. The TTV signals of these planet pairs will be significantly enhanced by their nearresonant orbits (Holman \& Murray 2005; Lithwick \& Wu 2012). 
Table 12

Planet Properties, K2-16

\begin{tabular}{|c|c|c|c|}
\hline Parameter & Planet $b$ & Planet c & Units \\
\hline \multicolumn{4}{|c|}{ Transit Model } \\
\hline$T_{0}$ & $811.6871 \pm 0.0038$ & $809.4800 \pm 0.0091$ & $\begin{array}{c}\mathrm{BJD}_{\mathrm{TDB}}- \\
2,456,000\end{array}$ \\
\hline$P$ & $7.61880 \pm 0.00087$ & $19.07863 \pm 0.00327$ & days \\
\hline$i$ & $87.97_{-1.86}^{+1.47}$ & $87.83 \pm 1.68$ & deg \\
\hline$R_{p} / R_{\star}$ & $0.02796_{-0.00259}^{+0.00363}$ & $0.03526_{-0.00650}^{+0.01553}$ & \\
\hline$R_{\star} / a$ & $0.0525_{-0.0127}^{+0.0268}$ & $0.0439_{-0.0210}^{+0.0285}$ & \\
\hline$u$ & $0.71 \pm 0.05$ & $0.72 \pm 0.05$ & \\
\hline$b$ & $0.68_{-0.43}^{+0.18}$ & $0.86_{-0.46}^{+0.07}$ & \\
\hline$t_{14}$ & $2.487_{-0.225}^{+0.382}$ & $3.859_{-0.728}^{+1.640}$ & \\
\hline$R_{p}$ & $2.02 \pm 0.24$ & $2.54_{-0.47}^{+1.12}$ & $R_{\oplus}$ \\
\hline$\rho_{\star, \operatorname{circ}}$ & $2.25_{-1.59}^{+2.90}$ & $0.61_{-0.48}^{+3.71}$ & $\mathrm{~g} \mathrm{~cm}^{-3}$ \\
\hline \multicolumn{4}{|c|}{ Derived Properties } \\
\hline$a$ & $0.0667 \pm 0.0011$ & $0.1229 \pm 0.0021$ & $\mathrm{AU}$ \\
\hline$S_{\text {inc }}$ & $44.5 \pm 5.2$ & $13.1 \pm 1.5$ & $S_{\oplus}$ \\
\hline$T_{\mathrm{eq}}$ & $658 \pm 19$ & $485 \pm 14$ & \\
\hline
\end{tabular}

Note. Same footnotes as Table 6.
Table 13

Planet Properties, K2-24

\begin{tabular}{|c|c|c|c|}
\hline Parameter & Planet b & Planet c & Units \\
\hline \multicolumn{4}{|c|}{ Transit Model } \\
\hline$T_{0}$ & $905.7950 \pm 0.0007$ & $915.6250 \pm 0.0005$ & $\begin{array}{c}\mathrm{BJD}_{\mathrm{TDB}} \\
2,456,000\end{array}$ \\
\hline$P$ & $20.88508 \pm 0.00036$ & $42.36342 \pm 0.00063$ & days \\
\hline$i$ & $88.95 \pm 0.62$ & $89.43_{-0.17}^{+0.26}$ & deg \\
\hline$R_{p} / R_{\star}$ & $0.04409 \pm 0.00146$ & $0.06147 \pm 0.00122$ & \\
\hline$R_{\star} / a$ & $0.0388_{-0.0041}^{+0.0062}$ & $0.0224 \pm 0.0017$ & \\
\hline$u$ & $0.56 \pm 0.03$ & $0.57 \pm 0.02$ & \\
\hline$b$ & $0.47_{-0.27}^{+0.16}$ & $0.44_{-0.18}^{+0.09}$ & \\
\hline$t_{14}$ & $5.881_{-0.187}^{+0.269}$ & $7.058 \pm 0.179$ & \\
\hline$R_{p}$ & $5.83 \pm 0.60$ & $8.10 \pm 0.82$ & $R_{\oplus}$ \\
\hline$\rho_{\star, \text { circ }}$ & $0.74 \pm 0.28$ & $0.94_{-0.18}^{+0.27}$ & $\mathrm{~g} \mathrm{~cm}^{-3}$ \\
\hline \multicolumn{4}{|c|}{ Derived Properties } \\
\hline$a$ & $0.1542 \pm 0.0026$ & $0.2471 \pm 0.0041$ & $\mathrm{AL}$ \\
\hline$S_{\text {inc }}$ & $60.1 \pm 12.4$ & $23.4 \pm 4.8$ & $S_{\theta}$ \\
\hline$T_{\mathrm{eq}}$ & $709 \pm 36$ & $560 \pm 29$ & \\
\hline
\end{tabular}

Note. Same footnotes as Table 6.

Table 14

Planet Properties, K2-37

\begin{tabular}{|c|c|c|c|c|}
\hline Parameter & Planet b & Planet c & Planet d & Units \\
\hline \multicolumn{5}{|c|}{ Transit Model } \\
\hline$T_{0}$ & $893.7013 \pm 0.0080$ & $898.8603 \pm 0.0023$ & $907.2315 \pm 0.0031$ & $\mathrm{BJD}_{\mathrm{TDB}}-2,456,000$ \\
\hline$P$ & $4.44117 \pm 0.00075$ & $6.42904 \pm 0.00036$ & $14.09189 \pm 0.00135$ & days \\
\hline$R_{p} / R_{\star}$ & $0.01728_{-0.00109}^{+0.00188}$ & $0.02955_{-0.00187}^{+0.00312}$ & $0.02950 \pm 0.00351$ & \\
\hline$R_{\star} / a$ & $0.0878_{-0.0135}^{+0.0420}$ & $0.0739_{-0.0147}^{+0.0340}$ & $0.0382_{-0.0123}^{+0.0259}$ & \\
\hline$u$ & $0.64 \pm 0.05$ & $0.64 \pm 0.05$ & $0.64 \pm 0.05$ & \\
\hline$R_{p}$ & $1.61 \pm 0.17$ & $2.75 \pm 0.27$ & $2.73 \pm 0.36$ & $R_{\oplus}$ \\
\hline$\rho_{\star, \mathrm{circ}}$ & $1.42 \pm 0.95$ & $1.13_{-0.77}^{+1.07}$ & $1.71_{-1.34}^{+3.80}$ & $\mathrm{~g} \mathrm{~cm}^{-3}$ \\
\hline \multicolumn{5}{|c|}{ Derived Properties } \\
\hline$a$ & $0.0511 \pm 0.0009$ & $0.0654 \pm 0.0011$ & $0.1103 \pm 0.0018$ & $\mathrm{AU}$ \\
\hline$S_{\text {inc }}$ & $213.3 \pm 27.8$ & $130.3 \pm 16.9$ & $45.7 \pm 6.0$ & $S_{\oplus}$ \\
\hline
\end{tabular}

Note. Same footnotes as Table 6.

\subsection{Comparison with Other Studies}

Table 17 compares our measured planet radii and host star radii with those published in other studies. All measurements agree within $1 \sigma$. Montet et al. (2015b) derive the radii of all 11 stars from photometry, yet their quoted uncertainties are often comparable to or smaller than our spectroscopic constraints. For example, for the two reddest stars (K2-3 and K2-5) they estimate uncertainties $\lesssim 2 \%$. They interpolate Dartmouth stellar evolution models, which, as the authors acknowledge, might systematically underestimate $\mathrm{M}$ dwarf radii by as much as $15 \%$ (Montet et al. 2015a; Newton et al. 2015). Moreover, below $\sim 0.8 M_{\odot}$, the scatter relative to precisely measured stellar radii is $\sim 4 \%$ (Feiden \& Chaboyer 2012). For the nine hotter stars, uncertainties from our stellar characterization algorithm Specmatch are well-calibrated large samples of exquisitely characterized stars (Petigura 2015). Therefore, we believe that our typical $\sim 5 \%-10 \%$ uncertainties are appropriate. Although the photometric derived uncertainties of Montet et al. (2015b) agree with our measurements, as both studies acknowledge, one should be cautious of adopting them for other analyses.

We thank Sam Grunblatt, Matthew Hosek Jr., John Livingston, and Geoff Marcy for helpful discussions. We thank Lauren Weiss and Lea Hirsch for their help with 
observing with Keck-HIRES. E.S. is supported by a postgraduate scholarship from the Natural Sciences and Engineering Research Council of Canada (NSERC). E.A.P. acknowledges support from a Hubble Fellowship grant HSTHF2-51365.001-A awarded by the Space Telescope Science Institute, which is operated by the Association of Universities for Research in Astronomy, Inc., for NASA under contract NAS 5-26555. A.W.H. acknowledges support for our $K 2$ team through a NASA Astrophysics Data Analysis Program grant. A.W.H. and I.J.M.C. acknowledge support from the $K 2$ Guest Observer Program. E.D.L. received funding from the European Union Seventh Framework Programme (FP7/2007-2013) under grant agreement no. 313014 (ETAEARTH). The research of J.E.S. was supported by an appointment to the NASA Postdoctoral Program at NASA Ames Research Center, administered by Oak Ridge Associated Universities through a

Table 15

Planet Properties, K2-38

\begin{tabular}{|c|c|c|c|}
\hline Parameter & Planet b & Planet c & Units \\
\hline \multicolumn{4}{|c|}{ Transit Model } \\
\hline$T_{0}$ & $896.8786 \pm 0.0054$ & $900.4752 \pm 0.0033$ & $\begin{array}{c}\mathrm{BJD}_{\mathrm{TDB}}- \\
2,456,000\end{array}$ \\
\hline$P$ & $4.01593 \pm 0.00050$ & $10.56103 \pm 0.00090$ & days \\
\hline$i$ & $87.28_{-3.08}^{+1.88}$ & $88.61_{-1.67}^{+1.00}$ & deg \\
\hline$R_{p} / R_{\star}$ & $0.01281_{-0.00064}^{+0.00105}$ & $0.02004_{-0.00135}^{+0.00236}$ & $\cdots$ \\
\hline$R_{\star} / a$ & $0.0993_{-0.0117}^{+0.0340}$ & $0.0381_{-0.0079}^{+0.0234}$ & $\cdots$ \\
\hline$u$ & $0.62 \pm 0.05$ & $0.61 \pm 0.05$ & $\cdots$ \\
\hline$b$ & $0.48 \pm 0.30$ & $0.64_{-0.41}^{+0.23}$ & $\cdots$ \\
\hline$t_{14}$ & $2.861 \pm 0.220$ & $2.533_{-0.144}^{+0.312}$ & $\mathrm{hr}$ \\
\hline$R_{p}$ & $1.55 \pm 0.16$ & $2.42 \pm 0.29$ & $R_{\oplus}$ \\
\hline$\rho_{\star, \mathrm{circ}}$ & $1.20_{-0.70}^{+0.55}$ & $3.06 \pm 2.71$ & $\mathrm{~g} \mathrm{~cm}^{-3}$ \\
\hline \multicolumn{4}{|c|}{ Derived Properties } \\
\hline$a$ & $0.0506 \pm 0.0008$ & $0.0964 \pm 0.0016$ & $\mathrm{AU}$ \\
\hline$S_{\text {inc }}$ & $465.9 \pm 80.1$ & $128.3 \pm 22.1$ & $S_{\oplus}$ \\
\hline$T_{\mathrm{eq}}$ & $1184 \pm 51$ & $858 \pm 37$ & K \\
\hline
\end{tabular}

Note. Same footnotes as Table 6 . contract with NASA. B.J.F. acknowledges support from a National Science Foundation Graduate Research Fellowship under grant no. 2014184874. This research used resources of the National Energy Research Scientific Computing Center, a DOE Office of Science User Facility supported by the Office of Science of the U.S. Department of Energy under Contract no. DE-AC02-05CH11231. This work made use of the SIMBAD database (operated at CDS, Strasbourg, France), NASA's Astrophysics Data System Bibliographic Services, and data products from the Two Micron All Sky Survey (2MASS), the APASS database, the SDSS-III project, and the Digitized Sky Survey. Some of the data presented in this paper were obtained from the Mikulski Archive for Space Telescopes (MAST). Support for MAST for non-HST data is provided by the NASA Office of Space Science via grant NNX09AF08G and by other grants and contracts. This research was made possible through the use of the AAVSO Photometric All-Sky Survey (APASS), funded by the Robert Martin Ayers Sciences Fund. This study benefits from use of the Robo-AO system, which was developed by collaborating partner institutions, the California Institute of Technology, and the Inter-University Centre for Astronomy and Astrophysics, and with the support of the National Science Foundation under grant nos. AST-0906060, AST-0960343, and AST-1207891, the Mt. Cuba Astronomical Foundation, and by a gift from Samuel Oschin. Ongoing science operation support of Robo-AO is provided by the California Institute of Technology and the University of Hawai'i. C.B. acknowledges support from the Alfred P. Sloan Foundation. Some of the data presented herein were obtained at the W. M. Keck Observatory (which is operated as a scientific partnership among Caltech, UC, and NASA). The authors wish to recognize and acknowledge the very significant cultural role and reverence that the summit of Maunakea has always had within the indigenous Hawaiian community. We are most fortunate to have the opportunity to conduct observations from this mountain.

Facilities: Kepler, K2, Keck:I (HIRES), Keck:II (NIRC2), IRTF (Spex), Palomar:Hale (PALM-3000/PHARO), PO:1.5 m (Robo-AO).

Table 16

Planet Properties, K2-32

\begin{tabular}{|c|c|c|c|c|}
\hline Parameter & Planet b & Planet c & Planet d & Units \\
\hline \multicolumn{5}{|c|}{ Transit Model } \\
\hline$T_{0}$ & $900.9258 \pm 0.0009$ & $899.4306 \pm 0.0101$ & $903.7846 \pm 0.0031$ & $\mathrm{BJD}_{\mathrm{TDB}}-2,456,000$ \\
\hline$P$ & $8.99218 \pm 0.00020$ & $20.65614 \pm 0.00598$ & $31.71922 \pm 0.00236$ & days \\
\hline$i$ & $89.00_{-0.90}^{+0.69}$ & $88.23_{-2.68}^{+1.32}$ & $88.40_{-0.65}^{+1.06}$ & deg \\
\hline$R_{p} / R_{\star}$ & $0.05635_{-0.00111}^{+0.00243}$ & $0.03636_{-0.00384}^{+0.01024}$ & $0.04004_{-0.00474}^{+0.00279}$ & $\cdots$ \\
\hline$R_{\star} / a$ & $0.0526_{-0.0029}^{+0.0078}$ & $0.0420_{-0.0132}^{+0.0427}$ & $0.0355 \pm 0.0114$ & $\cdots$ \\
\hline$u$ & $0.66 \pm 0.04$ & $0.66 \pm 0.05$ & $0.66 \pm 0.05$ & $\ldots$ \\
\hline$b$ & $0.33 \pm 0.22$ & $0.74_{-0.46}^{+0.18}$ & $0.79_{-0.37}^{+0.07}$ & $\cdots$ \\
\hline$t_{14}$ & $3.693_{-0.105}^{+0.193}$ & $5.024_{-0.492}^{+2.307}$ & $5.990 \pm 0.729$ & $\mathrm{hr}$ \\
\hline$R_{p}$ & $5.38 \pm 0.35$ & $3.48_{-0.42}^{+0.97}$ & $3.75 \pm 0.40$ & $R_{\oplus}$ \\
\hline$\rho_{\star, \operatorname{circ}}$ & $1.61_{-0.54}^{+0.30}$ & $0.60_{-0.52}^{+1.24}$ & $0.42_{-0.22}^{+1.15}$ & $\mathrm{~g} \mathrm{~cm}^{-3}$ \\
\hline \multicolumn{5}{|c|}{ Derived Properties } \\
\hline$a$ & $0.0808 \pm 0.0013$ & $0.1407 \pm 0.0024$ & $0.1873 \pm 0.0031$ & $\mathrm{AU}$ \\
\hline$S_{\text {inc }}$ & $82.9 \pm 10.6$ & $27.4 \pm 3.5$ & $15.4 \pm 2.0$ & $S_{\oplus}$ \\
\hline$T_{\text {eq }}$ & $769 \pm 25$ & $583 \pm 19$ & $505 \pm 16$ & $\mathrm{~K}$ \\
\hline
\end{tabular}

Note. Same footnotes as Table 6. 
Table 17

Comparison with Other Studies

\begin{tabular}{|c|c|c|c|c|c|}
\hline EPIC & $R_{p}$ (This Study) & $R_{p}$ (Other) & $R_{\star}$ (This Study) & $R_{\star}($ Other $)$ & References \\
\hline $201338508 b$ & $1.91 \pm 0.44$ & $1.92 \pm 0.23$ & $0.57 \pm 0.12$ & $0.52 \pm 0.01$ & M15 \\
\hline $201338508 \mathrm{c}$ & $2.26 \pm 0.62$ & $1.92 \pm 0.20$ & $0.57 \pm 0.12$ & $0.52 \pm 0.01$ & M15 \\
\hline $201367065 b$ & $2.18 \pm 0.30$ & $1.98 \pm 0.10,2.14 \pm 0.27$ & $0.56 \pm 0.07$ & $0.52 \pm 0.02,0.56 \pm 0.07$ & M15, C15 \\
\hline $201367065 d$ & $1.51 \pm 0.23$ & $1.52 \pm 0.21$ & $0.56 \pm 0.07$ & $0.56 \pm 0.07$ & C15 \\
\hline $201445392 b$ & $3.58 \pm 0.71$ & $2.97 \pm 0.51$ & $0.74 \pm 0.04$ & $0.74_{-0.03}^{+0.02}$ & M15 \\
\hline 201445392c & $2.41 \pm 0.33$ & $2.31 \pm 0.33$ & $0.74 \pm 0.04$ & $0.74_{-0.03}^{+0.02}$ & M15 \\
\hline $201549860 \mathrm{c}$ & $2.09_{-0.24}^{+0.33}$ & $2.20 \pm 0.40$ & $0.72 \pm 0.04$ & $0.69 \pm 0.02$ & M15 \\
\hline $201754305 b$ & $2.02 \pm 0.24$ & $2.13 \pm 0.37$ & $0.66 \pm 0.03$ & $0.64 \pm 0.03$ & M15 \\
\hline $201754305 \mathrm{c}$ & $2.54_{-0.47}^{+1.12}$ & $2.14 \pm 0.41$ & $0.66 \pm 0.03$ & $0.64 \pm 0.03$ & M15 \\
\hline $203771098 b$ & $5.83 \pm 0.60$ & $5.68 \pm 0.41$ & $1.21 \pm 0.12$ & $1.21 \pm 0.11$ & P16 \\
\hline $203771098 \mathrm{c}$ & $8.10 \pm 0.82$ & $7.82 \pm 0.72$ & $1.21 \pm 0.12$ & $1.21 \pm 0.11$ & P16 \\
\hline
\end{tabular}

References. M15-Montet et al. (2015b); C15—Crossfield et al. (2015); A15—Armstrong et al. (2015); B15—Barros et al. (2015); P16—Petigura et al. (2016).

\section{REFERENCES}

Adams, E. R., Seager, S., \& Elkins-Tanton, L. 2008, ApJ, 673, 1160 Agol, E., Steffen, J., Sari, R., \& Clarkson, W. 2005, MNRAS, 359, 567 Ahn, C. P., Alexandroff, R., Allende Prieto, C., et al. 2012, ApJS, 203, 21 Almenara, J. M., Astudillo-Defru, N., Bonfils, X., et al. 2015, A\&A, 581, L7 Armstrong, D. J., Santerne, A., Veras, D., et al. 2015, A\&A, 582, A33 Baranec, C., Riddle, R., Law, N. M., et al. 2013, Journal of Visualized Experiments, 72, e50021

Baranec, C., Riddle, R., Law, N. M., et al. 2014, ApJL, 790, L8

Barros, S. C. C., Almenara, J. M., Demangeon, O., et al. 2015, MNRAS, 454,4267

Batalha, N. M., Rowe, J. F., Bryson, S. T., et al. 2013, ApJS, 204, 24

Batygin, K., \& Morbidelli, A. 2013, AJ, 145, 1

Beichman, C., Livingston, J., Werner, M., et al. 2016, ApJ, 822, 39

Borucki, W. J., Koch, D. G., Basri, G., et al. 2011, ApJ, 736, 19

Boyajian, T. S., von Braun, K., van Belle, G., et al. 2012, ApJ, 757, 112

Burke, C. J., Bryson, S. T., Mullally, F., et al. 2014, ApJS, 210, 19

Butler, R. P., Marcy, G. W., Williams, E., et al. 1996, PASP, 108, 500

Carter, J. A., Agol, E., Chaplin, W. J., et al. 2012, Sci, 337, 556

Ciardi, D. R., Beichman, C. A., Horch, E. P., \& Howell, S. B. 2015, ApJ, 805,16

Claret, A., Hauschildt, P. H., \& Witte, S. 2012, A\&A, 546, A14

Claret, A., Hauschildt, P. H., \& Witte, S. 2013, A\&A, 552, A16

Coelho, P., Barbuy, B., Meléndez, J., Schiavon, R. P., \& Castilho, B. V. 2005, A\&A, 443, 735

Crossfield, I. J. M., Ciardi, D. R., Petigura, E. A., et al. 2016, arXiv:1607. 05263

Crossfield, I. J. M., Petigura, E., Schlieder, J. E., et al. 2015, ApJ, 804, 10

Cushing, M. C., Rayner, J. T., \& Vacca, W. D. 2005, ApJ, 623, 1115

Cushing, M. C., Vacca, W. D., \& Rayner, J. T. 2004, PASP, 116, 362

Dai, F., Winn, J. N., Albrecht, S., et al. 2016, ApJ, 823, 115

Dekany, R., Roberts, J., Burruss, R., et al. 2013, ApJ, 776, 130

Dotter, A., Chaboyer, B., Jevremović, D., et al. 2008, ApJS, 178, 89

Dressing, C. D., Charbonneau, D., Dumusque, X., et al. 2015, ApJ, 800, 135

Fabrycky, D. C., Lissauer, J. J., Ragozzine, D., et al. 2014, ApJ, 790, 146

Fang, J., \& Margot, J.-L. 2012, ApJ, 761, 92

Fang, J., \& Margot, J.-L. 2013, ApJ, 767, 115

Feiden, G. A., \& Chaboyer, B. 2012, ApJ, 757, 42

Foreman-Mackey, D., Hogg, D. W., Lang, D., \& Goodman, J. 2013, PASP, 125,306

Foreman-Mackey, D., Montet, B. T., Hogg, D. W., et al. 2015, ApJ, 806, 215

Fressin, F., Torres, G., Charbonneau, D., et al. 2013, ApJ, 766, 81

Gelman, A., \& Rubin, D. B. 1992, StaSc, 7, 457

Gladman, B. 1993, Icar, 106, 247

Hansen, B. M. S., \& Murray, N. 2012, ApJ, 751, 158

Holman, M. J., \& Murray, N. W. 2005, Sci, 307, 1288

Howard, A. W., Johnson, J. A., Marcy, G. W., et al. 2009, ApJ, 696, 75

Howard, A. W., Johnson, J. A., Marcy, G. W., et al. 2010, ApJ, 721, 1467

Howard, A. W., Marcy, G. W., Bryson, S. T., et al. 2012, ApJS, 201, 15
Howard, A. W., Marcy, G. W., Fischer, D. A., et al. 2014, ApJ, 794, 51

Howell, S. B., Sobeck, C., Haas, M., et al. 2014, PASP, 126, 398

Isaacson, H., \& Fischer, D. 2010, ApJ, 725, 875

Kaiser, N., Burgett, W., Chambers, K., et al. 2010, Proc. SPIE, 7733, 0

Kolbl, R., Marcy, G. W., Isaacson, H., \& Howard, A. W. 2015, AJ, 149, 18

Kraus, A. L., \& Hillenbrand, L. A. 2007, AJ, 134, 2340

Law, N. M., Morton, T., Baranec, C., et al. 2014, ApJ, 791, 35

Liddle, A. R. 2007, MNRAS, 377, L74

Lissauer, J. J., Fabrycky, D. C., Ford, E. B., et al. 2011a, Natur, 470, 53

Lissauer, J. J., Marcy, G. W., Bryson, S. T., et al. 2014, ApJ, 784, 44

Lissauer, J. J., Marcy, G. W., Rowe, J. F., et al. 2012, ApJ, 750, 112

Lissauer, J. J., Ragozzine, D., Fabrycky, D. C., et al. 2011b, ApJS, 197, 8

Lithwick, Y., \& Wu, Y. 2012, ApJL, 756, L11

Mann, A. W., Gaidos, E., \& Ansdell, M. 2013a, ApJ, 779, 188

Mann, A. W., Gaidos, E., Kraus, A., \& Hilton, E. J. 2013b, ApJ, 770, 43

Marcus, R. A., Sasselov, D., Hernquist, L., \& Stewart, S. T. 2010, ApJL, 712, L73

Marcy, G. W., \& Butler, R. P. 1992, PASP, 104, 270

Meunier, N., Desort, M., \& Lagrange, A.-M. 2010, A\&A, 512, A39

Middelkoop, F. 1982, A\&A, 107, 31

Montet, B. T., Johnson, J. A., Muirhead, P. S., et al. 2015a, ApJ, 800, 134

Montet, B. T., Morton, T. D., Foreman-Mackey, D., et al. 2015b, ApJ, 809, 25

Morton, T. D. 2015a, Isochrones: Stellar model grid package, Astrophysics Source Code Library, ascl:1503.010

Morton, T. D. 2015b, VESPA: False positive probabilities calculator, Astrophysics Source Code Library, ascl:1503.011

Morton, T. D., \& Johnson, J. A. 2011, ApJ, 738, 170

Mullally, F., Coughlin, J. L., Thompson, S. E., et al. 2015, ApJS, 217, 31

Narita, N., Hirano, T., Fukui, A., et al. 2015, ApJ, 815, 47

Newton, E. R., Charbonneau, D., Irwin, J., \& Mann, A. W. 2015, ApJ, 800, 85

Noyes, R. W., Hartmann, L. W., Baliunas, S. L., Duncan, D. K., \& Vaughan, A. H. 1984, ApJ, 279, 763

Petigura, E. A. 2015a, PhD thesis, Univ. California

Petigura, E. A., Howard, A. W., \& Marcy, G. W. 2013, PNAS, 110, 19273

Petigura, E. A., Howard, A. W., Lopez, E. D., et al. 2016, ApJ, 818, 36

Petigura, E. A., \& Marcy, G. W. 2012, PASP, 124, 1073

Petigura, E. A., Schlieder, J. E., Crossfield, I. J. M., et al. 2015b, ApJ, 811, 102

Rayner, J. T., Cushing, M. C., \& Vacca, W. D. 2009, ApJS, 185, 289

Rayner, J. T., Toomey, D. W., Onaka, P. M., et al. 2003, PASP, 115, 362

Rojas-Ayala, B., Covey, K. R., Muirhead, P. S., \& Lloyd, J. P. 2012, ApJ, 748, 93

Rowe, J. F., Bryson, S. T., Marcy, G. W., et al. 2014, ApJ, 784, 45

Rowe, J. F., Coughlin, J. L., Antoci, V., et al. 2015, ApJS, 217, 16

Schmitt, J. R., Wang, J., Fischer, D. A., et al. 2014, AJ, 148, 28

Schwarz, G. 1978, AnSta, 6, 461

Seager, S., Kuchner, M., Hier-Majumder, C. A., \& Militzer, B. 2007, ApJ, 669,1279

Southworth, J. 2011, MNRAS, 417, 2166

Southworth, J., Maxted, P. F. L., \& Smalley, B. 2004, MNRAS, 351, 1277 
Terrien, R. C., Mahadevan, S., Bender, C. F., et al. 2012, ApJL, 747, L38 Vacca, W. D., Cushing, M. C., \& Rayner, J. T. 2003, PASP, 115, 389

Valencia, D., Guillot, T., Parmentier, V., \& Freedman, R. S. 2013, ApJ, 775,10

Valenti, J. A., Butler, R. P., \& Marcy, G. W. 1995, PASP, 107, 966

Vanderburg, A., Latham, D. W., Buchhave, L. A., et al. 2016, ApJS, 222, 14

Vanderburg, A., Montet, B. T., Johnson, J. A., et al. 2015, ApJ, 800, 59

Vogt, S. S., Allen, S. L., Bigelow, B. C., et al. 1994, Proc. SPIE, 2198, 362
Weiss, L. M., \& Marcy, G. W. 2014, ApJL, 783, L6

Wilson, O. C. 1968, ApJ, 153, 221

Winn, J. N., Johnson, J. A., Howard, A. W., et al. 2010, ApJ, 718, 575

Wolfgang, A., Rogers, L. A., \& Ford, E. B. 2015, arXiv:1504.07557

Wright, J. T. 2005, PASP, 117, 657

Wright, J. T., \& Howard, A. W. 2009, ApJS, 182, 205

Wurm, G., Trieloff, M., \& Rauer, H. 2013, ApJ, 769, 78

Zeng, L., \& Sasselov, D. 2013, PASP, 125, 227 